TRANSACTIONS OF THE

AMERICAN MATHEMATICAL SOCIETY

Volume 350, Number 9, September 1998, Pages 3409-3482

S $0002-9947(98) 02121-7$

\title{
ON THE SUBGROUP STRUCTURE OF EXCEPTIONAL GROUPS OF LIE TYPE
}

\author{
MARTIN W. LIEBECK AND GARY M. SEITZ
}

\begin{abstract}
We study finite subgroups of exceptional groups of Lie type, in particular maximal subgroups. Reduction theorems allow us to concentrate on almost simple subgroups, the main case being those with socle $X(q)$ of Lie type in the natural characteristic. Our approach is to show that for sufficiently large $q$ (usually $q>9$ suffices), $X(q)$ is contained in a subgroup of positive dimension in the corresponding exceptional algebraic group, stabilizing the same subspaces of the Lie algebra. Applications are given to the study of maximal subgroups of finite exceptional groups. For example, we show that all maximal subgroups of sufficiently large order arise as fixed point groups of maximal closed subgroups of positive dimension.
\end{abstract}

\section{INTRODUCTION}

In this paper we establish results aimed at the study of the subgroup structure of the finite exceptional groups of Lie type, in particular of their maximal subgroups. Let $G(r)$ be an exceptional group of Lie type over a finite field $\mathbb{F}_{r}$ of order $r$, where $r$ is a power of the prime $p$. Write $G(r)=O^{p^{\prime}}\left(G_{\sigma}\right)$, where $G$ is a simple algebraic group over $\overline{\mathbb{F}}_{r}$ and $\sigma$ is a Frobenius morphism of $G$.

The reduction theorem [LS1, Theorem 2] determines all maximal subgroups of $G(r)$ which are not almost simple. Thus we concentrate on almost simple maximal subgroups of $G(r)$; let $M$ be such a subgroup. Of particular interest is the so-called "generic case", in which $F^{*}(M)=X(q)$, a group of Lie type over a field $\mathbb{F}_{q}$, also of characteristic $p$.

Our approach to the generic case is to attempt to show that the embedding $X(q)<G(r)$ lifts to an embedding $\bar{X} \leq G$, where $\bar{X}$ is a simple closed connected subgroup of $G$ of the same type as $X(q)$. Once this is achieved, we are in a position to apply results from [Se2, LS1] on the subgroup structure of algebraic groups.

The first lifting result for exceptional groups appeared in [ST1], and showed that the finite embedding can be lifted under certain hypotheses, one of the hypotheses being that the characteristic $p$ be suitably large. In this paper we establish lifting results in which there are hypotheses on the size of the ground field $\mathbb{F}_{q}$, but no assumptions on the characteristic. Consequently, the results hold for all but finitely many possibilities for the group $X(q)$. For example, Theorem 6 below shows that for $q$ sufficiently large, either $X(q)$ is the group of fixed points of an automorphism of

Received by the editors October 11, 1996.

1991 Mathematics Subject Classification. Primary 20G40, 20 E28.

The authors acknowledge the support of NATO Collaborative Research Grant CRG 931394. The second author also acknowledges the support of an NSF Grant. 
$G(r)$, or $X(q)=O^{p^{\prime}}\left(\bar{X}_{\sigma}\right)$ with $\bar{X}$ maximal among closed connected $M\langle\sigma\rangle$-invariant subgroups of $G$. (In most cases, "sufficiently large" means that $q>9$.)

Once the lifting has been achieved we can apply the results of [Se2, LS1], which determine those subgroups $\bar{X}$ of $G$ as in the last sentence of the previous paragraph, under some mild characteristic restrictions. These restrictions are much weaker than those required for the result in [ST1]; in particular, $p=0$ or $p>7$ covers all the restrictions. However, in view of the results obtained in this paper, it becomes a high priority issue to extend the analysis in [Se2, LS1] so as to remove the characteristic restrictions. Such an analysis is under way.

The preceding discussion has been couched in terms of maximal subgroups, but our results apply much more generally. We establish lifting results for all subgroups $X(q)$ of $G(r)$, with $q$ sufficiently large. Namely, in Theorem 1 we show that any such subgroup is contained in a closed connected subgroup $\bar{X}$ of $G$ stabilizing precisely the same subspaces of the Lie algebra $L(G)$ as $X(q)$. We also show (Theorem 4 and Corollary 5) that this connected group $\bar{X}$ is almost always proper in $G$, and that, given mild characteristic restrictions, $X(q)=O^{p^{\prime}}\left(\bar{X}_{\delta}\right)$ for some Frobenius morphism $\delta$ (Theorem 10). At this point the results of [LS2] determine the conjugacy class of $X(q)$ in $G(r)$.

Finally, we use our lifting results to clear up a nagging problem on classical groups. In Theorem 11, we show that an embedding $X<Y<C$ of finite groups of Lie type in the same characteristic, with $C$ classical and $X$ absolutely irreducible on the usual module for $C$, can usually be lifted to a corresponding embedding of simple algebraic groups; such embeddings of algebraic groups are known [Se1]. See [Se3] for further discussion of this problem.

We now state our results in detail, beginning with our main lifting results. As indicated above, these require no assumption on the characteristic $p$, but do need an assumption on $q$, where $X=X(q)$ is as above. In order to specify this assumption we need the following definition.

Definition. Let $G$ be a simple adjoint algebraic group, and let $\Sigma=\Sigma(G)$ be the root system of $G$. For a subgroup $L$ of the lattice $\mathbb{Z} \Sigma$, let $t(L)$ be the exponent of the torsion subgroup of $\mathbb{Z} \Sigma / L$. For $\alpha, \beta \in \Sigma$, call the element $\alpha-\beta$ of $\mathbb{Z} \Sigma$ a root difference. Define

$$
t(\Sigma(G))=\max \{t(L): L \text { a subgroup of } \mathbb{Z} \Sigma \text { generated by root differences }\} .
$$

Theorem 1. Let $X=X(q)$ be a quasisimple group of Lie type in characteristic $p$, and suppose that $X<G$, where $G$ is a simple adjoint algebraic group of exceptional type, also in characteristic $p$. Assume that

$$
\begin{aligned}
q>t(\Sigma(G)) .(2, p-1) & \text { if } X=A_{1}(q),{ }^{2} B_{2}(q) \text { or }{ }^{2} G_{2}(q), \\
q>9 \text { and } X \neq A_{2}^{\epsilon}(16) & \text { otherwise. }
\end{aligned}
$$

Then the following hold:

(i) there is a closed connected subgroup $\bar{X}$ of $G$ containing $X$, such that every $X$-invariant subspace of the Lie algebra $L(G)$ is also $\bar{X}$-invariant;

(ii) if also $X \leq G_{\sigma}$ where $\sigma$ is a Frobenius morphism, then there is a $\sigma$-stable and $N_{G}(X)$-stable subgroup $\bar{X}$ containing $X$ such that each $X$-invariant subspace of each $G$-composition factor of $L(G)$ is also $\bar{X}$-invariant.

Theorem 4 below will show that the subgroup $\bar{X}$ in the conclusion is proper in $G$, unless $X$ has the same type as $G$. 
Remark. If $p$ is a good prime for $G$, then $L(G)$ is irreducible as a $G$-module, so the subspace invariance conditions in conclusions (i) and (ii) are equivalent; however, for bad primes $L(G)$ can be reducible (see Proposition 1.10 below), in which case (i) is stronger than (ii).

In view of Theorem 1 , it is of interest to know the values of $t(\Sigma(G))$, particularly for groups $G$ of exceptional type. Dr Ross Lawther has informed us that he has verified (using a computer) that $t\left(G_{2}\right)=12, t\left(F_{4}\right)=68, t\left(E_{6}\right)=124$ and $t\left(E_{7}\right)=$ 388 , while $t\left(E_{8}\right)$ is unknown at present.

The bounds in Theorem 1 for groups of type $A_{1},{ }^{2} B_{2},{ }^{2} G_{2}$ are relatively large, and follow from Corollary 3 below, which we shall deduce from the following general proposition. It should be possible to improve these bounds with additional work.

Proposition 2. Let $G$ be a simple adjoint algebraic group, and let $x$ be a semisimple element of $G$ of finite order greater than $\operatorname{mt}(\Sigma(G))$, for some positive integer $m$. Then there is an infinite closed subgroup $S$ of $G$ such that the following both hold:

(i) $x \in S$, and some nontrivial power of $x$, of order greater than $m$, lies in $S^{0}$;

(ii) every $x$-invariant subspace of $L(G)$ is also $S$-invariant.

Corollary 3. Let $X=X(q)$ be a quasisimple group of Lie type in characteristic $p$, and suppose that $X<G$, where $G$ is a simple adjoint algebraic group, also in characteristic $p$. Assume that $q>t(\Sigma(G)) m(X)$, where $m(X)$ is the order of the Schur multiplier of $X / Z(X)$. Then conclusions (i) and (ii) of Theorem 1 hold.

Note that Proposition 2 and Corollary 3 apply to all types of simple algebraic group, not just exceptional types.

The proofs of Proposition 2 and Corollary 3 are rather short, and are given in $\S 2$. Reducing the bounds on $q$ to those in Theorem 1 requires a great deal more effort. After some preliminary results in $\S 3$, this is carried out for $p \neq 2$ in $\S \S 4,5$ and in $\S 6$ for $p=2$. With some further effort, our proof could no doubt be extended to improve the $q>9$ bound for subgroups $X$ of rank larger than 2; indeed, when $X$ has rank greater than half the rank of $G$, [LST, Theorem 2] gives a stronger conclusion assuming only that $q>2$.

In order to make use of Theorem 1 (or Corollary 3), we need to know that the subgroup $\bar{X}$ is proper in $G$. For this it suffices to show that $\bar{X}$ acts reducibly on some $G$-composition factor of $L(G)$. This information is provided by the next result.

Definition. We define $X$ and $G$ to be of the same type if $X \cong G_{\sigma}^{(\infty)}$ for some Frobenius morphism $\sigma$; we also say that $X$ is a group of type $G$.

Theorem 4. Let $G$ be a simple algebraic group in characteristic $p$, and let $X=$ $X(q)$ be a subgroup of $G$, where $q=p^{e}$ and $X$ is quasisimple. Suppose that $X$ is not of the same type as $G$, and that $X$ acts irreducibly on every $G$-composition factor of $L(G)$.

Then $(G, p)=\left(C_{n}, 2\right)$ or $\left(B_{n}, 2\right)$, and either $X=D_{n}^{\epsilon}(q)$ or $n=3$ and $X=$ $G_{2}(q)$; further, $X$ lies in a simple connected subgroup $D_{n}$ or $G_{2}$ of $G$.

Combining Theorems 1 and 4, we immediately have

Corollary 5. Let $G$ be a simple adjoint algebraic group of exceptional type, and suppose that $X=X(q)<G$, where $q$ is as in the hypotheses of Theorem 1. Then 
either $X$ is of the same type as $G$, or $X<\bar{X}<G$, where $\bar{X}$ is closed and connected, and leaves invariant every $X$-invariant subspace of $L(G)$.

Using this result we shall derive Theorem 6 below, which concerns the maximal subgroups of the finite exceptional groups of Lie type. In the statement we refer to [LS1, Theorem 1]; in this result, certain positive integers $N(\bar{X}, G)$ are defined for pairs $(\bar{X}, G)$ of simple algebraic groups, as follows:

\begin{tabular}{r|rrrrr} 
& $G=E_{8}$ & $E_{7}$ & $E_{6}$ & $F_{4}$ & $G_{2}$ \\
\hline$X=A_{1}$ & 7 & 7 & 5 & 3 & 3 \\
$A_{2}$ & 5 & 5 & 3 & 3 & \\
$B_{2}, G_{2}$ & 5 & 3 & 3 & 2 & \\
$B_{3}$ & 2 & 2 & 2 & & \\
$A_{3}, C_{3}, B_{4}$ & 2 & & & & \\
\hline
\end{tabular}

For example, $N\left(A_{2}, E_{7}\right)=5$, and so on. For $(\bar{X}, G)$ not in the table, set $N(\bar{X}, G)=$ 1. We define $N(X(q), G)$ to be equal to $N(\bar{X}, G)$, where $\bar{X}$ is a simple algebraic group of the same type as $X(q)$.

The statement of Theorem 6 and its corollaries involves groups $G, L$ and $L_{1}$ such that $G$ is a simple adjoint algebraic group of exceptional type in characteristic $p, \sigma$ is a Frobenius morphism such that $L=O^{p^{\prime}}\left(G_{\sigma}\right)$ is a finite simple group of exceptional Lie type, and $L_{1}$ is a finite group with socle $L$ (i.e. $L \leq L_{1} \leq$ Aut $L$ ).

Theorem 6. Let $L=O^{p^{\prime}}\left(G_{\sigma}\right)$ and $L \leq L_{1} \leq$ Aut $L$, as above. Suppose that $H$ is an almost simple maximal subgroup of $L_{1}$, and $F^{*}(H)=X(q)$ with $q$ satisfying the hypotheses of Theorem 1. Then one of the following holds:

(i) $X(q)$ has the same type as $G$;

(ii) $X(q)=O^{p^{\prime}}\left(\bar{X}_{\sigma}\right)$ for some maximal closed connected reductive $H\langle\sigma\rangle$-stable subgroup $\bar{X}$ of $G$. (The subgroup $\bar{X}$ is given by [LS1, Theorem 1] if $\bar{X}$ is non-simple or if $p>N(X(q), G)$.)

Remarks. 1. There is no assumption on $p$ in the theorem.

2. The subgroups satisfying (i) are uniquely determined up to $G_{\sigma}$-conjugacy by [LS2, 5.1].

3. Our proof of Theorem 6 shows, more generally, that if $H$ is any (not necessarily maximal) subgroup of $L_{1}$ such that $F^{*}(H)=X(q)$ with $q$ as in Theorem 1 , then $H \leq N\left(\bar{X}_{\sigma}\right)$ for some $\bar{X}$ as in conclusion (ii).

4. In our proof of Theorem 6 we do not require the full force of the hypothesis on $q$; indeed, in the proof we only require the existence of a subgroup $\bar{X}$ satisfying the conclusions of Theorem 1.

We recall one further definition before stating the next result. If $G, L, L_{1}$ are as above, and $D$ is a $\sigma$-stable connected reductive subgroup of $G$ of maximal rank (i.e. containing a maximal torus), then we call $N_{L_{1}}\left(D_{\sigma}\right)$ a subgroup of maximal rank of $L_{1}$. The subgroups of maximal rank which are maximal in $L_{1}$ are determined in [LSS].

Corollary 7. Let $L=O^{p^{\prime}}\left(G_{\sigma}\right)$ and $L \leq L_{1} \leq$ Aut $L$, as above. Let $H$ be an almost simple maximal subgroup of $L_{1}$, with $F^{*}(H)=X(q)$ and $q$ as in Theorem 1 , and let $G_{\sigma}=G\left(q_{1}\right)$. Then one of the following holds:

(i) $H$ is a subgroup of maximal rank;

(ii) $X(q)$ has the same type as $G$; 
(iii) $q=q_{1}$ and $X(q)=O^{p^{\prime}}\left(\bar{X}_{\sigma}\right)$, where $\bar{X}$ is a simple maximal connected $\sigma$ stable subgroup of $G$ not containing a maximal torus. Moreover, assuming that $p>N(X(q), G)$, the possibilities are:

$G=G_{2}: \quad X(q)=A_{1}(q)(p \geq 7)$,

$G=F_{4}: \quad X(q)=A_{1}(q)(p \geq 13)$ or $G_{2}(q)(p=7)$,

$G=E_{6}: \quad X(q)=A_{2}^{\epsilon}(q)(\epsilon= \pm, p \geq 5), G_{2}(q)(p \geq 5, p \neq 7), F_{4}(q)$ or $C_{4}(q)(p \neq 2)$,

$G=E_{7}: \quad X(q)=A_{1}(q)(2$ classes, $p \geq 17,19)$ or $A_{2}^{\epsilon}(q)(\epsilon= \pm, p \geq 7)$,

$G=E_{8}: \quad X(q)=A_{1}(q)(3$ classes, $p \geq 23,29,31)$ or $B_{2}(q)(p \geq 7)$.

Assuming $p>N(X(q), G)$, there is just one $\operatorname{Aut}(G)$-class of subgroups $\bar{X}$ of each isomorphism type occurring, and each such $\operatorname{Aut}(G)$-class gives a unique $\operatorname{Aut}\left(G_{\sigma}\right)$ class of subgroups $X(q)$.

Corollary 8. Let $L=O^{p^{\prime}}\left(G_{\sigma}\right)$ and $L \leq L_{1} \leq$ Aut $L$, as above. There is a constant c (independent of $G, L, L_{1}$ ), such that if $H$ is a maximal subgroup of $L_{1}$ with $|H|>c$, then either

(i) $H$ is the normalizer of a subgroup of the same type as $G$, or

(ii) $H=N_{L_{1}}\left(\bar{X}_{\sigma}\right)$ for some maximal closed connected $H\langle\sigma\rangle$-stable subgroup $\bar{X}$ of $G$.

Next we state our results on embeddings of arbitrary subgroups $X(q)$ of an exceptional adjoint algebraic group $G$. Theorem 9 is the analogue of [LS2, Theorem 1] for finite groups. In the statement we use numbers $N^{\prime}(X, G)$ as defined in [LS2]; these are roughly the same as the numbers $N(X, G)$, with a few differences. The precise definition of $N^{\prime}(X, G)$ is given by the following table:

\begin{tabular}{r|rrrrr} 
& $G=E_{8}$ & $E_{7}$ & $E_{6}$ & $F_{4}$ & $G_{2}$ \\
\hline$X$ of type $A_{1}$ & 7 & 7 & 5 & 3 & 3 \\
$A_{2}$ & 5 & 5 & 3 & 3 & \\
$B_{2}$ & 5 & 3 & 3 & 2 & \\
$G_{2}$ & 7 & 7 & 3 & 2 & \\
$B_{3}$ & 2 & 2 & 2 & 2 & \\
$C_{3}$ & 3 & 2 & 2 & 2 & \\
$A_{3}, B_{4}, C_{4}, D_{4}$ & 2 & 2 & 2 & &
\end{tabular}

Theorem 9. Let $X=X(q)<G$, with $G$ of exceptional type and $q$ as in Theorem 1 , and assume that $p>N^{\prime}(X, G)$. If $X$ lies in a parabolic subgroup $Q L$ of $G$, with unipotent radical $Q$ and Levi subgroup $L$, then $X$ lies in a $Q$-conjugate of $L$. Further, if also $X<G_{\sigma}$ (where $\sigma$ is a Frobenius morphism), and $Q L$ is $\sigma$-stable, then $X$ lies in a $\sigma$-stable $Q$-conjugate of $L$.

Theorem 10. Let $X=X(q)<G$, with $G$ of exceptional type and $q$ as in Theorem 1 , and assume that $p>N^{\prime}(X, G)$. Then

(i) $X$ lies in a closed connected simple subgroup $\tilde{X}$ of $G$ of the same type as $X$, and $C_{G}(X)^{0}=C_{G}(\tilde{X})^{0}$;

(ii) if also $X<G_{\sigma}$ with $\sigma$ a Frobenius morphism of $G$, then $X$ lies in a closed connected semisimple $\sigma$-stable subgroup $\bar{X}=X_{1} \ldots X_{t}$ of $G$, where each factor $X_{i}$ is simple of the same type as $X$; moreover, $C_{G}(X)^{0}=C_{G}(\bar{X})^{0}$.

The embeddings of the subgroups $\tilde{X}$ and $\bar{X}$ in the conclusion of Theorem 10 are given by [LS2, Theorems 5 and 7], so Theorem 10 determines the embeddings of $X(q)$ in $G$ and $G_{\sigma}$. 
For $X=X(q)$ of rank more than $\frac{1}{2} \operatorname{rank}(G)$, the conclusion of Theorem 10 is obtained in [LST, Theorem 2] with no assumption on $p$, and assuming only that $q>2$.

Finally, we give a consequence of Theorem 1 to the study of subgroups of finite classical groups.

Theorem 11. Let $C$ be a finite classical simple group in characteristic $p$, with usual module $V$. Suppose that $X=X(q)$ and $Y$ are finite simple groups of Lie type in characteristic $p$, not of the same type, such that $X<Y<C$ and $X$ is absolutely irreducible on $V$; when $Y$ is of exceptional type, assume also that $q$ is as in Theorem 1 (relative to $Y$ ).

Then the embedding $X<Y<C$ lifts to an embedding of closed subgroups $\bar{X}<\bar{Y} \leq \bar{C}$, where $\bar{X}, \bar{Y}, \bar{C}$ are connected simple algebraic groups of the same types as $X, Y, C$.

Remark. Assuming $\bar{Y}, \bar{C}$ are not both classical groups on the same natural module, all possibilities for the triple $(\bar{X}, \bar{Y}, \bar{C})$ in the conclusion are given by [Se1, Te1].

The layout of the paper is as follows. After the preliminary first section, we give in $\S 2$ the proofs of Proposition 2 and Corollary 3. The following four sections are devoted to the proof of Theorem 1 . In $\S 3$ we describe the strategy and give some preliminary lemmas. The proof is then carried out for $q$ odd in $\S \S 4,5$, and for $q$ even in $\S 6$. In $\S 7$ we prove Theorem 4 , and $\S 8$ contains proofs of Theorem 6 and its corollaries. Theorems 9 and 10 are proved in $\S 9$, and Theorem 11 in $\S 10$.

We use the following notation throughout the paper. If $Y$ is a connected simple algebraic group over the algebraically closed field $K$, or a corresponding finite group of Lie type, and $\lambda$ is a dominant weight, then $V_{Y}(\lambda)$ denotes the rational irreducible $K Y$-module with high weight $\lambda$, and $W_{Y}(\lambda)$ denotes the corresponding Weyl module. If $V_{1}, \ldots, V_{k}$ are modules, then $V_{1} / \ldots / V_{k}$ denotes a module having the same composition factors as $V_{1} \oplus \ldots \oplus V_{k}$. We often abbreviate this notation as follows: if $\mu_{1}, \ldots, \mu_{k}$ are dominant weights for the group $Y$, and $c_{1}, \ldots, c_{k}$ are positive integers, then

$$
\mu_{1}^{c_{1}} / \ldots / \mu_{k}^{c_{k}}
$$

always denotes a $K Y$-module having the same composition factors as the module $V_{Y}\left(\mu_{1}\right)^{c_{1}} \oplus \ldots \oplus V_{Y}\left(\mu_{k}\right)^{c_{k}}$.

When $Y$ is a subgroup of $G$ and $V$ is a $K G$-module, we use $V \downarrow Y$ for the restriction of $V$ to $Y$. If $Y$ is connected and simple in characteristic $p>0$, and $q$ is a power of $p$, then $\sigma_{q}$ denotes a standard Frobenius morphism of $Y$; that is, a morphism inducing the map $x_{\alpha}(t) \rightarrow x_{\alpha}\left(t^{q}\right)$ on root groups. If $V$ is a $K Y$-module, we write $V^{(q)}$ for the $K Y$-module obtained from $V$ by twisting the action of $Y$ by $\sigma_{q}$ (i.e. changing the action from $v \rightarrow v y$ to $v \rightarrow v y^{\sigma_{q}}$ ).

We use $T_{i}$ to denote a torus of rank $i$, and $W(G)$ for the Weyl group of $G$. The fundamental roots in a fundamental system for $G$ are denoted $\alpha_{1}, \ldots, \alpha_{l}$, and the corresponding fundamental dominant weights are $\lambda_{1}, \ldots, \lambda_{l}$. The Dynkin diagram of $G$ is labelled as in [Bo, p.250].

For $\epsilon= \pm, A_{n}^{\epsilon}(q)$ denotes the group $A_{n}(q)$ if $\epsilon=+$ and ${ }^{2} A_{n}(q)$ if $\epsilon=-$; we use similar notation for types $D_{n}^{\epsilon}, E_{6}^{\epsilon}, B_{2}^{\epsilon}, G_{2}^{\epsilon}, F_{4}^{\epsilon}$, and also for $D_{4}^{\epsilon}$ we allow $\epsilon=3$ to denote ${ }^{3} D_{4}$. Finally, if $X$ is a finite group of Lie type, then $\operatorname{rk}(X)$ denotes the rank of the simple algebraic group corresponding to $X$. 


\section{Preliminaries}

In this section we present some preliminary results which will be used throughout the paper.

The first few results concern representations of groups of type $A_{1}$. For the group $G=S L_{2}(K)$ over the algebraically closed field $K$ of characteristic $p>0$, and for $0 \leq i \leq p-1$, we denote simply by $i$ (or sometimes $V(i)$ ) the rational irreducible $K G$-module with high weight $i \lambda_{1}$, of dimension $i+1$; these are the restricted $K G$ modules. By Steinberg's tensor product theorem, every finite-dimensional rational irreducible $K G$-module is a tensor product $i_{0} \otimes i_{1}^{(p)} \otimes \ldots \otimes i_{k}^{\left(p^{k}\right)}$ of field twists of restricted modules $i_{0}, \ldots, i_{k}$, and such a module has high weight $\sum i_{r} p^{r}$.

Using [St, Lemma 79], we have the following well known result.

Proposition 1.1. Let $V(i)$ denote the restricted module for $G=S L_{2}(K)$ of high weight $i$. If $i$ is odd, then the induced group

$$
G^{V(i)}=S L_{2}(K) \leq S p(V(i))
$$

and if $i$ is even, then $G^{V(i)}=P S L_{2}(K) \leq S O(V(i))$.

The next result concerns extensions of modules for $S L_{2}(K)$ and its subgroups $S L_{2}\left(p^{e}\right)$, and is taken from [AJL, 3.9 and 4.5]. We thank Professor J-P. Serre for pointing out to us that [AJL, 4.5] has a small omission when $e=2$, and giving us the corrected version below.

Proposition 1.2. Let $G=S L_{2}(K)$, and for $e \geq 1$ denote by $G_{e}$ a subgroup $S L_{2}\left(p^{e}\right)$ of $G$.

(i) Suppose $p>2$ and let $0 \leq a, b \leq p-1$. Then $\operatorname{Ext}_{G_{1}}^{1}(a, b) \neq 0$ if and only if $a+b=p-3$ or $p-1$.

(ii) Let $e \geq 2$, and suppose $\lambda, \mu<p^{e}$ have $p$-adic expansions $\lambda=\sum_{0}^{e-1} a_{i} p^{i}, \mu=$ $\sum_{0}^{e-1} b_{i} p^{i}$ (where $0 \leq a_{i}, b_{i} \leq p-1$ ). Set $a_{e}=a_{0}$ and $b_{e}=b_{0}$. Then $\operatorname{Ext}_{G_{e}}^{1}(\lambda, \mu) \neq 0$ if and only if there exists $k \geq 0$ such that

$$
a_{i}=b_{i} \text { for } i \notin\{k, k+1\}, a_{k}+b_{k}=p-2 \text {, and } a_{k+1}-b_{k+1}= \pm 1 .
$$

Further, $\operatorname{Ext}_{G_{e}}^{1}(\lambda, \mu)$ has dimension at most 1 , except if $e=2$ and, writing $s=$ $\frac{1}{2}(p-1), t=\frac{1}{2}(p-3)$, we have $\left(a_{0}, a_{1}, b_{0}, b_{1}\right)=(s, s, t, t),(t, t, s, s),(s, t, t, s)$ or $(t, s, s, t)$ (in which case it has dimension 2 ).

(iii) Let $\lambda, \mu$ be positive integers with $p$-adic expansions $\lambda=\sum a_{i} p^{i}, \mu=\sum b_{i} p^{i}$. Then $\operatorname{Ext}_{G}^{1}(\lambda, \mu) \neq 0$ if and only if there exists $k \geq \nu_{p}(\lambda+1)$ (where $\nu_{p}(\lambda+1)=$ $\max \left\{i: p^{i}\right.$ divides $\left.\left.\lambda+1\right\}\right)$, such that

$$
a_{i}=b_{i} \text { for } i \notin\{k, k+1\}, a_{k}+b_{k}=p-2 \text {, and } a_{k+1}-b_{k+1}= \pm 1 .
$$

Further, $\operatorname{Ext}_{G}^{1}(\lambda, \mu)$ has dimension at most 1 .

Proposition 1.3. Let $G=S L_{2}(K), G_{e}=S L_{2}\left(p^{e}\right)$, and let $0 \leq \lambda, \mu<p^{e}$.

(i) The restriction map $\operatorname{Ext}_{G}^{1}(\lambda, \mu) \rightarrow \operatorname{Ext}_{G_{e}}^{1}(\lambda, \mu)$ is injective; indeed, this map is an isomorphism unless either $e=1$, or $\lambda, \mu$ are as in the exceptional cases at the end of 1.2(ii).

(ii) Suppose that $e \geq 2$, and, if $e=2$, that $\lambda$, $\mu$ are not as in the exceptional cases at the end of 1.2(ii). Then there exist $\lambda^{\prime}, \mu^{\prime}$ such that $\lambda^{\prime} \downarrow G_{e} \cong \lambda \downarrow G_{e}$, 
$\mu^{\prime} \downarrow G_{e} \cong \mu \downarrow G_{e}$ and

$$
\operatorname{Ext}_{G}^{1}\left(\lambda^{\prime}, \mu^{\prime}\right) \cong \operatorname{Ext}_{G_{e}}^{1}\left(\lambda^{\prime}, \mu^{\prime}\right) \cong \operatorname{Ext}_{G_{e}}^{1}(\lambda, \mu) .
$$

In particular, taking $\mu=0$, we have $H^{1}\left(G, \lambda^{\prime}\right) \cong H^{1}\left(G_{e}, \lambda^{\prime}\right) \cong H^{1}\left(G_{e}, \lambda\right)$.

Proof. Part (i) is immediate from [CPSK, 7.4], and part (ii) follows from 1.2; note that in (ii) it may be necessary to choose $\lambda^{\prime} \neq \lambda$ or $\mu^{\prime} \neq \mu$, since by 1.2 there exist $\lambda, \mu$ such that $\operatorname{Ext}_{G}^{1}(\lambda, \mu)=0$ but $\operatorname{Ext}_{G_{e}}^{1}(\lambda, \mu) \neq 0$ (the pairs $\lambda, \lambda^{\prime}$ and $\mu, \mu^{\prime}$ are related by a field morphism of $G$ ).

Proposition 1.4. Let $G$ be a connected simple algebraic group over $K$ and let $H$ be a finite subgroup of $G$. Suppose $V$ is a finite-dimensional rational $K G$-module satisfying the following conditions:

(i) every $G$-composition factor of $V$ is $H$-irreducible;

(ii) for any $G$-composition factors $M, N$ of $V$, the restriction map $\operatorname{Ext}_{G}^{1}(M, N) \rightarrow$ $\operatorname{Ext}_{H}^{1}(M, N)$ is injective;

(iii) for any $G$-composition factors $M, N$ of $V$, if $M \downarrow H \cong N \downarrow H$ then $M \cong N$ (as $G$-modules).

Then $G$ and $H$ fix exactly the same subspaces of $V$.

Proof. The proof goes by induction on $\operatorname{dim} V$. It is clearly sufficient to show that if $W$ is an irreducible $H$-submodule of $V$, then $G$ fixes $W$.

Thus let $W$ be an irreducible $H$-submodule of $V$. Define $U=\left\langle W^{G}\right\rangle$, and suppose that $W \neq U$. If $U$ is $G$-irreducible, then by (i) it is $H$-irreducible, so $U=W$, contrary to assumption. Therefore $U$ is $G$-reducible; let $W_{0}$ be a $G$-irreducible submodule of $U$. Then $W_{0} \neq W$.

Consider $V / W_{0}$. Now $H$ fixes the subspace $\left(W+W_{0}\right) / W_{0}$ of this, which is $H$ irreducible. By induction, $G$ fixes $W+W_{0}$. Hence $U=W+W_{0}$. As $W, W_{0}$ are $H$-irreducible, $U=W+W_{0}=W \oplus W_{0}$. Thus $U$ is not $H$-indecomposable, and so by (ii), $U$ is also not $G$-indecomposable. Therefore there is a $G$-module $W_{1}$ such that

$$
U=W \oplus W_{0}=W_{1} \oplus W_{0} .
$$

Note that $W$ is $H$-isomorphic to $W_{1}$. If $W$ is not $H$-isomorphic to $W_{0}$, then $W, W_{0}$ are the only irreducible $H$-submodules of $U$, so $W_{1}=W$ and $G$ fixes $W$, a contradiction. Therefore $W$ is $H$-isomorphic to $W_{0}$, and hence by (iii) these two spaces are also $G$-isomorphic. Now $W \subseteq W_{1} \oplus W_{0}$, so $W=\left\{w+w \phi: w \in W_{1}\right\}$ for some $H$-isomorphism $\phi: W_{1} \rightarrow W_{0}$. But $\phi$ is a $G$-isomorphism: for let $\alpha$ be any $G$-isomorphism from $W_{1}$ to $W_{0}$. Then $\alpha \phi^{-1}: W_{1} \rightarrow W_{1}$ is an $H$-isomorphism, so by Schur's Lemma, $\alpha \phi^{-1}=\lambda$.id for some $\lambda \in K^{*}$. Hence $\phi=\lambda^{-1} \alpha$, and so $\phi$ is a $G$-isomorphism, as claimed. It follows that $W$ is fixed by $G$, contrary to our assumption that $W \neq U$. Therefore $W=U$, and this completes the proof.

The next result is a consequence of 1.4; it is actually a special case of [CPSK, 7.5].

Corollary 1.5. Let $G=S L_{2}(K)$ and $G_{e}=S L_{2}\left(p^{e}\right)<G$. If $V$ is a finitedimensional rational $K G$-module such that every composition factor of $V \downarrow G$ has high weight less than $p^{e}$, then $G$ and $G_{e}$ fix exactly the same subspaces of $V$.

Proof. Take $H=G_{e}$ in 1.4. Conditions (i) and (iii) of 1.4 are clearly satisfied, and condition (ii) follows from 1.3(i). 
Proposition 1.6. Let $G=S L_{2}(K)$ and $Y \cong S L_{2}\left(p^{e}\right)$, and let $T, T_{Y}$ be Cartan subgroups of $G, Y$ respectively. Let $Q$ be a finite-dimensional rational $K G$-module, and regard the semidirect product $Q G$ as an algebraic group. Suppose that $Y<Q G$, $T_{Y}<Q T, C_{Q}(T)=C_{Q}\left(T_{Y}\right)$ and that $H^{1}(G, Q) \cong H^{1}(Y, Q)$. Then there is a closed complement to $Q$ in $Q G$ which contains $Y$.

Proof. We may assume that $T_{Y}<T$. For $x \in Y$, let $x=u_{x} r_{x}$ with $u_{x} \in Q, r_{x} \in G$. Then $X=\left\{r_{x}: x \in Y\right\}$ is a subgroup of $G$ isomorphic to $Y$, and the hypotheses hold with $X$ replacing $Y$. The map $\gamma: X \rightarrow Q$ given by $\gamma\left(r_{x}\right)=u_{x}$ is a 1cocycle. The assumption that $C_{Q}(T)=C_{Q}\left(T_{Y}\right)$ implies that the restriction map $H^{1}(G, Q) \rightarrow H^{1}(X, Q)$ is injective, by [CPSK, 7.3]. Hence by hypothesis, the restriction map is an isomorphism, and $\gamma$ extends to a rational 1-cocycle $\bar{\gamma}: G \rightarrow Q$. Then $\{\bar{\gamma}(g) g: g \in G\}$ is a closed complement to $Q$ in $Q G$ containing $Y$.

Proposition 1.7. Let $G=S O_{2 n}(K)$, where $K$ is algebraically closed of characteristic $p>2 n$, and let $u$ be an element of $G$ of order $p$. Then there is a closed connected subgroup $A \cong A_{1}(K)$ of $G$ containing $u$, such that every composition factor of $V_{G}\left(\lambda_{1}\right) \downarrow A$ is restricted.

Proof. Let $V=V_{G}\left(\lambda_{1}\right)$. By [SS, IV, 2.14 and 2.15], $u$ is determined up to $G$ conjugacy by the sizes of its Jordan blocks on $V$; moreover, the number of Jordan blocks of each even size is even. Let $r_{1}, \ldots, r_{k}$ be the sizes of the odd Jordan blocks of $u$, and $s_{1}, s_{1}, \ldots, s_{l}, s_{l}$ the sizes of the even Jordan blocks, listed in pairs. Then $G$ has a subgroup $\prod_{1}^{k} S O_{r_{i}} \times \prod_{1}^{l} S L_{s_{i}}$. Each factor has an irreducible subgroup of type $A_{1}(K)$, and we define $A$ to be a diagonal subgroup of the product of these $A_{1}$ 's such that the composition factors of $V \downarrow A$ have high weights $r_{i}-1, s_{i}-1$. Any nontrivial unipotent element of $A$ has the same Jordan block sizes on $V$ as $u$, hence is $G$-conjugate to $u$. This completes the proof.

Next we state two results on modules for groups of type $A_{2}$. The first is taken from [LS2, 1.9, 1.11], and is a consequence of the sum formula in [An].

Proposition 1.8. Let $G=A_{2}(K)$, and let $a, b \in\{0,1, \ldots, p-1\}$.

(i) If $a+b+2 \leq p$, or if $b=0$, then $W_{G}(a b)$ is irreducible.

(ii) If $W_{G}(a b)$ is reducible, then it is indecomposable with two composition factors, $V_{G}(a b)$ and $V_{G}(p-b-2, p-a-2)$.

(iii) $W_{G}(a b)$ has dimension $\frac{1}{2}(a+1)(b+1)(a+b+2)$.

The next result is a straightforward application of 1.8 .

Proposition 1.9. Let $A=A_{2}^{\epsilon}(p)$ with $p=11$ or 13 , and let $S$ be a subgroup $S L_{2}(p)$ of $A$ centralizing an involution. Suppose $V=V_{A}(a b)$ is a restricted module for A satisfying

(i) $a \geq b$ and $a+b \geq 9$, and

(ii) $\operatorname{dim} V \leq 124$ if $a \neq b$, and $\operatorname{dim} V \leq 248$ if $a=b$.

Then the possibilities for $a, b$ and $V \downarrow S$ are as in the following table (in the last column, the superscripts denote the multiplicities of each composition factor). 


\begin{tabular}{lccl}
$a b$ & $p$ & $\operatorname{dim} V$ & $V \downarrow S$ \\
\hline 12,0 & 13 & 91 & $12^{1} / 11^{1} / 10^{1} / \ldots$ \\
11,0 & 13 & 78 & $11^{1} / 10^{1} / 9^{1} / \ldots$ \\
10,0 & 11,13 & 66 & $10^{1} / 9^{1} / 8^{1} / \ldots$ \\
90 & 11,13 & 55 & $9^{1} / 8^{1} / 7^{1} / \ldots$ \\
11,1 & 13 & 102 & $12^{1} / 11^{2} / 10^{1} / \ldots$ \\
91 & 13 & 120 & $10^{1} / 9^{2} / \ldots$ \\
& 11 & 75 & $10^{1} / 9^{2} / 8^{2} / \ldots$ \\
81 & 11,13 & 99 & $9 / 8^{2} / \ldots$. \\
10,2 & 13 & 111 & $12^{1} / 11^{2} / 10^{2} / \ldots$ \\
82 & 11 & 82 & $10^{1} / 9^{2} / 8^{2} / 7^{1} \ldots$ \\
93 & 13 & 118 & $12^{1} / 11^{2} / 10^{2} / 9^{2} / \ldots$ \\
73 & 11 & 87 & $10^{1} / 9^{2} / 8^{2} / \ldots$ \\
84 & 13 & 123 & $12^{1} / 11^{2} / 10^{2} / \ldots$ \\
66 & 13 & 127 & $12^{1} / 11^{2} / 10^{2} / \ldots$ \\
64 & 11 & 90 & $10^{1} / 9^{2} / 8^{2} / \ldots$ \\
55 & 13 & 216 & $10^{1} / 9^{2} / 8^{3} / \ldots$ \\
& 11 & 91 & $10^{1} / 9^{2} / 8^{2} / 7^{2} \ldots$ \\
\hline
\end{tabular}

Next we prove some results concerning the representation of a simple algebraic group $G$ on its Lie algebra $L(G)$. The first is well known, but we give a proof for completeness.

Proposition 1.10. The $G$-composition factors of $L(G)$ are as in the following table.

\begin{tabular}{ccll}
$G$ & $p$ & $\begin{array}{l}\text { comp. factors } \\
\text { of } L(G)\end{array}$ & dimensions \\
\hline$A_{n}$ & $p \nmid n+1$ & $\lambda_{1}+\lambda_{n}$ & $(n+1)^{2}-1$ \\
& $p \mid n+1$ & $\lambda_{1}+\lambda_{n} / 0$ & $(n+1)^{2}-2,1$ \\
$B_{n}$ & $p \neq 2$ & $\lambda_{2}$ & $2 n^{2}+n$ \\
& $p=2, n$ odd & $\lambda_{1} / \lambda_{2} / 0$ & $2 n, 2 n^{2}-n-1,1$ \\
& $p=2, n>2$ even & $\lambda_{1} / \lambda_{2} / 0^{2}$ & $2 n, 2 n^{2}-n-2,2$ \\
& $p=2, n=2$ & $\lambda_{1} / 2 \lambda_{2} / 0^{2}$ & $4,4,2$ \\
$C_{n}$ & $p \neq 2$ & $2 \lambda_{1}$ & $2 n^{2}+n$ \\
& $p=2, n$ odd & $2 \lambda_{1} / \lambda_{2} / 0$ & $2 n, 2 n^{2}-n-1,1$ \\
& $p=2, n$ even & $2 \lambda_{1} / \lambda_{2} / 0^{2}$ & $2 n, 2 n^{2}-n-2,2$ \\
$D_{n}$ & $p \neq 2$ & $\lambda_{2}$ & $2 n^{2}-n$ \\
& $p=2, n$ odd & $\lambda_{2} / 0$ & $2 n^{2}-n-1,1$ \\
$E_{8}$ & $p=2, n$ even & $\lambda_{2} / 0^{2}$ & $2 n^{2}-n-2,2$ \\
$E_{7}$ & any & $\lambda_{8}$ & 248 \\
& $p \neq 2$ & $\lambda_{1}$ & 133 \\
$E_{6}$ & $p=2$ & $\lambda_{1} / 0$ & 132,1 \\
& $p \neq 3$ & $\lambda_{2}$ & 78 \\
$F_{4}$ & $p=3$ & $\lambda_{2} / 0$ & 77,1 \\
& $p \neq 2$ & $\lambda_{1}$ & 52 \\
$G_{2}$ & $p=2$ & $\lambda_{1} / \lambda_{4}$ & 26,26 \\
& $p \neq 3$ & $\lambda_{2}$ & 14 \\
\hline
\end{tabular}


Proof. For $G$ of exceptional type this is proved in $[\mathrm{Se} 2,1.2]$. Assume now that $G$ is classical.

Consider first $G=A_{n}$. The highest root vector in $L(G)$ affords weight $\lambda_{1}+\lambda_{n}$, so the composition factor $V_{G}\left(\lambda_{1}+\lambda_{n}\right)$ of $L(G)$ has all $n^{2}+n$ roots occuring as weights. By $\left[\right.$ Se1, 8.6], the 0 -weight space of $V_{G}\left(\lambda_{1}+\lambda_{n}\right)$ has dimension $n$ if $p \nmid n+1$, and dimension $n-1$ if $p \mid n$. The conclusion follows for $A_{n}$.

Next consider $G=B_{n}, C_{n}, D_{n}$ with $p \neq 2$. The highest long root vector affords weight $\lambda_{2}, 2 \lambda_{1}, \lambda_{2}$ respectively; hence $L(G)$ has a composition factor of this high weight. Also, if $V=V_{G}\left(\lambda_{1}\right)$ (of dimension $2 n+1,2 n, 2 n$ respectively), then by [Se1, 8.1], the $G$-modules $\bigwedge^{2} V, S^{2} V, \bigwedge^{2} V$ (respectively) are irreducible, and are isomorphic to $V_{G}\left(\lambda_{2}\right), V_{G}\left(2 \lambda_{1}\right), V_{G}\left(\lambda_{2}\right)$. Since $\bigwedge^{2} V, S^{2} V, \bigwedge^{2} V$ (respectively) have dimension equal to that of $L(G)$, it follows that $L(G)$ is irreducible of high weight $\lambda_{2}, 2 \lambda_{1}, \lambda_{2}$ respectively, as in the conclusion.

In all the remaining cases we have $p=2$. We begin with $G=D_{n}$. We may take $n \geq 4$ and $G=S O_{2 n}=S O(V)$ (note that the composition factors of $L(G)$ are independent of the form of the group $G)$. The highest root vector affords weight $\lambda_{2}$. We work out the dimension of the composition factor $V_{G}\left(\lambda_{2}\right)$, by considering the action of $G$ on $\bigwedge^{2} V$ (of which $V_{G}\left(\lambda_{2}\right)$ is a section). Observe first that the orbit of $\lambda_{2}$ under the Weyl group of $G$ has size $2 n^{2}-2 n$ (which is the number of roots of $D_{n}$ ); so the dimension of $V_{G}\left(\lambda_{2}\right)$ will be determined once we calculate the dimension of the 0 -weight space. Now $G$ has a subgroup $G L_{n}$ containing a maximal torus $T$. The Weyl group $S_{n}$ of $G L_{n}$ acts on the 0 -weight space $M$ of $T$ on $\bigwedge^{2} V$ as on the natural $n$-dimensional permutation module over $K$. Writing $V_{i}$ for an irreducible $S_{n}$-module of dimension $i$, we have

$$
\begin{aligned}
M \downarrow S_{n}= & V_{1} \oplus V_{n-1}, \text { if } n \text { is odd } \\
& V_{1} / V_{n-2} / V_{1} \text { (indecomposable), if } n \text { is even. }
\end{aligned}
$$

A subgroup $G L_{4}$ of $G$ has a composition factor of high weight $\lambda_{1}+\lambda_{3}$ in $V_{G}\left(\lambda_{2}\right)$. Hence by the $A_{n}$ case above, we see that the 0 -weight space of $V_{G}\left(\lambda_{2}\right)$ has dimension at least 2 ; from the $S_{n}$-action described above, the 0 -weight space therefore has dimension at least $n-1$ if $n$ is odd, and at least $n-2$ if $n$ is even. Therefore $\operatorname{dim} V_{G}\left(\lambda_{2}\right)$ is at least $2 n^{2}-n-1$ if $n$ is odd, and at least $2 n^{2}-n-2$ if $n$ is even. As $G=S O(V)<S p(V)$, we see that $G$ fixes a 1 -space $\langle w\rangle$ in $\bigwedge^{2} V$. It follows that $\operatorname{dim} V_{G}\left(\lambda_{2}\right)=2 n^{2}-n-1$ if $n$ is odd. Finally, $\operatorname{dim} V_{G}\left(\lambda_{2}\right)=2 n^{2}-n-2$ if $n$ is even: for in this case the $G$-invariant 1-space $\langle w\rangle$ cannot be a direct summand (as $M \downarrow S_{n}$ is indecomposable), so the self-duality of $\bigwedge^{2} V$ implies that there is a $G$-invariant subspace $W$ of codimension 1 in $\bigwedge^{2} V$, and $V_{G}\left(\lambda_{2}\right) \cong W /\langle w\rangle$.

Next consider $G=B_{n}$, with $p=2$ and $n>2$. The highest long root affords weight $\lambda_{2}$, and the highest short root affords $\lambda_{1}$. Also $V_{G}\left(\lambda_{1}\right)$ has dimension $2 n$. Moreover, $G$ fixes the ideal $I$ of $L(G)$ generated by all $e_{\alpha}$ with $\alpha$ a short root; and $I \subseteq L\left(A_{1}^{n}\right)$, where $A_{1}^{n}$ is a commuting product of short $A_{1}$ 's. Thus a consideration of weights implies that $I \downarrow G=\lambda_{1} / 0^{k}$ for some $k$. Now the long roots form a subsystem $D_{n}$, so the composition factor $V_{D_{n}}\left(\lambda_{2}\right)$ of $L\left(D_{n}\right)$ is a section of $L(G) / I$. By $[\mathrm{Se} 1,4.1(\mathrm{iii})], V_{D_{n}}\left(\lambda_{2}\right)$ has the same dimension as $V_{B_{n}}\left(\lambda_{2}\right)$, and by the previous paragraph, this dimension is $2 n^{2}-n-1$ if $n$ is odd, and $2 n^{2}-n-2$ if $n$ is even. The conclusion now follows. The case where $G=B_{2}, p=2$ follows from the $C_{2}$ case, given below. 
Finally, let $G=C_{n}$ with $p=2$. The highest long root affords $2 \lambda_{1}$, and the highest short root affords $\lambda_{2}$. Again $G$ fixes the ideal of $L(G)$ generated by all $e_{\alpha}$ with $\alpha$ short, which is contained in $L\left(D_{n}\right)$. By [Se1, 4.1(iii)], $\operatorname{dim} V_{C_{n}}\left(\lambda_{2}\right)=\operatorname{dim} V_{D_{n}}\left(\lambda_{2}\right)$, and the conclusion follows as above.

Proposition 1.11. Let $G$ be a simple algebraic group in characteristic $p$, and let $T$ be a maximal torus of $G$. Then $C_{L(G)}(L(T))=L(T)$, unless $(G, p)=\left(C_{n}, 2\right)$ (or $\left.\left(B_{2}, 2\right)\right)$. In the exceptional cases, $C_{L(G)}(L(T))=L\left(A_{1}^{n}\right)$ (or $L\left(A_{1}^{2}\right)$ ).

Proof. Let $A=C_{L(G)}(L(T))$. Then $A$ is $T$-invariant, hence is spanned by $L(T)$, together with those elements $e_{\alpha}$ of $L(G)$ which centralize $L(T)$. Note that if $e_{\alpha} \in A$ then $e_{-\alpha} \in A$. We may assume that $G \neq A_{1}$.

Suppose $A \neq L(T)$, so that $e_{\alpha} \in A$ for some root $\alpha$. Conjugating by an element of the Weyl group, we may assume that $\alpha$ is a fundamental root. As $h_{\alpha}$ centralizes $e_{\alpha}$, we have $p=2$.

If there is a root $\beta$ such that $(\alpha, \beta)=-1$, then $h_{\beta}$ does not centralize $e_{\alpha}$, a contradiction. Therefore $\alpha$ cannot lie in an $A_{2}$ subsystem of $\Sigma(G)$. Hence there must be more than one root length, and $\alpha$ must be a long root. It follows that $G=B_{2}$ or $C_{n}$. The $B_{2}$ case can be treated as $C_{2}$, so we take $G=C_{n}$. Now the proof of 1.10 shows that the weight $\alpha$ occurs within a composition factor $V_{C_{n}}\left(2 \lambda_{1}\right)$ of $L(G)$. Because of the field twist, $L(T)$ acts trivially on this module. Moreover, the fundamental $A_{1}$ 's corresponding to long roots commute, so this completes the proof.

Proposition 1.12. Let $G$ be a simple algebraic group over $K$, and let $\phi: G \rightarrow G$ be a morphism which is an automorphism of abstract groups.

(i) Suppose that $G_{\phi}$ is not a finite Suzuki or Ree group, and let $V$ be a $G$ composition factor of $L(G)$. If $M$ is a subspace of $V$, then $\left(G_{M}\right)^{\phi}=G_{M^{\prime}}$ for some subspace $M^{\prime}$ of $V$.

(ii) Suppose $G_{\phi}$ is a finite Suzuki or Ree group, and let $V_{1}, V_{2}$ be the two $G$ composition factors of $L(G)$. If $M$ is a subspace of $V_{i}(i=1,2)$, then $\left(G_{M}\right)^{\phi}=G_{M^{\prime}}$ for some subspace $M^{\prime}$ of $V_{3-i}$.

(iii) Let $X$ be a $\phi$-stable subgroup of $G$, and let $\mathcal{M}$ be the collection of all $X$-invariant subspaces of all $G$-composition factors of $L(G)$. Then the subgroup $\bigcap_{W \in \mathcal{M}} G_{W}$ of $G$ is $\phi$-stable.

Proof. (i) Let the $G$-module $V$ have high weight $\lambda$ and correspond to the representation $\rho: G \rightarrow G L(V)$. We may write $\phi=y \tau \sigma$, where $y, \tau, \sigma$ are (possibly trivial) inner, graph, field automorphisms, respectively. By 1.10, the representations $\rho$ and $\tau \rho$ of $G$ are equivalent, as they both have high weight $\lambda$. Hence if $\sigma$ is a $q$-power field automorphism (where $q=p^{e} \geq 1$ ), then the high weight of the representation $\phi \rho$ is $q \lambda$. There is therefore a $q$-power field automorphism $\omega$ of $G L(V)$ such that the representations $\phi \rho$ and $\rho \omega$ of $G$ are equivalent. The automorphism $\omega$ is induced by a semilinear transformation $V \rightarrow V$ which we shall also denote by $\omega$. Then $y^{\omega}=\omega^{-1} y \omega$ for $y \in G L(V)$. Thus, identifying each $g \in G$ with its image $g \rho \in G L(V)$, there exists $x \in G L(V)$ such that $g^{\phi}=g^{\omega x}=x^{-1} \omega^{-1} g \omega x$, for all $g \in G$. Writing $\delta=\omega x$, this gives $\delta g^{\phi}=g \delta$ for all $g \in G$, and we have

$$
(v \delta) g^{\phi}=(v g) \delta
$$

for all $v \in V, g \in G$. If $M$ is a subspace of $V$, and $m \in M, g \in G_{M}$, then $(m \delta) g^{\phi}=(m g) \delta \in M \delta$, and hence $g^{\phi} \in G_{M \delta}$. Therefore $\left(G_{M}\right)^{\phi} \leq G_{M \delta}$. For the 
reverse inclusion, write $g \in G_{M \delta}$ as $g=\left(g^{\phi^{-1}}\right)^{\phi}$. Using the displayed equality, we see that $g^{\phi^{-1}} \in G_{M}$, as required. Part (i) is now established.

(ii) Here $G_{\phi}$ is a finite group of type ${ }^{2} B_{2},{ }^{2} G_{2}$ or ${ }^{2} F_{4}$. Let $V_{1}, V_{2}$ be the composition factors of $L(G)$, as in 1.10 , and let $\rho_{i}: G \rightarrow G L\left(V_{i}\right)(i=1,2)$ be the corresponding representations. As above, consideration of high weights shows that there are automorphisms $\omega_{i}$ of $G L\left(V_{i}\right)$ such that the representations $\phi \rho_{i}$ and $\rho_{3-i} \omega_{3-i}$ are equivalent for $i=1,2$. Thus there are invertible linear transformations $\delta: V_{1} \rightarrow V_{2}$, $\gamma: V_{2} \rightarrow V_{1}$ such that

$$
\left(v_{1} g^{\phi}\right) \delta=\left(v_{1} \delta\right) g^{\omega_{2}}, \quad\left(v_{2} g^{\phi}\right) \gamma=\left(v_{2} \gamma\right) g^{\omega_{1}}
$$

for all $v_{i} \in V_{i}, g \in G$ (where we write just $v_{i} g$ instead of $v_{i}\left(g \rho_{i}\right)$. A calculation similar to that in part (i) now shows that for subspaces $M_{i}$ of $V_{i}$, we have

$$
G_{M_{1}}=\left(G_{M_{1} \delta \omega_{2}^{-1}}\right)^{\phi}, G_{M_{2}}=\left(G_{M_{2} \gamma \omega_{1}^{-1}}\right)^{\phi}
$$

giving (ii).

Finally, (iii) is immediate from (i) and (ii).

The next result is presumably well known, but we have been unable to find a reference for it.

Proposition 1.13. Let $G$ be a simple algebraic group over $K=\overline{\mathbb{F}}_{p}$, and let $\sigma$ be a Frobenius morphism of $G$ such that $O^{p^{\prime}}\left(G_{\sigma}\right)=G(q)$, a finite group of Lie type over $\mathbb{F}_{q}$. Suppose $H$ is a closed, connected, simple subgroup of $G$ which is $\sigma$-stable. Then $O^{p^{\prime}}\left(H_{\sigma}\right)=H(q)$, a group of Lie type over the same field $\mathbb{F}_{q}$.

Proof. Assume first that $G_{\sigma}$ is not a Suzuki or Ree group, so that $\sigma$ is defined over $\mathbb{F}_{q}$. By [SS, I, 2.9], $\sigma$ fixes a Borel subgroup $B_{H}$ of $H$, and a maximal torus $T_{H}$ therein. Let $U_{H}=R_{u}\left(B_{H}\right)$. Notice that since $\sigma$ is defined over $\mathbb{F}_{q}, H_{\sigma}$ is also not a Suzuki or Ree group; hence there exists a $\sigma$-stable long root subgroup $U$ of $U_{H}$.

We claim that $\left|U_{\sigma}\right|=q$. To see this, observe first that since the action of $\sigma$ on $U \cong K^{+}$is defined over $\mathbb{F}_{q}$ (see [Bor1, AG 14.4]), we have $K[U]=K[x] \cong K \otimes \mathbb{F}_{q}[x]$. Hence the comorphism $\sigma^{*}$ induces the $q$-power map $x \rightarrow x^{q}$ on $\mathbb{F}_{q}[x]$. There is a polynomial $f$ such that $\sigma(u)=f(u)$ for all $u \in U$, and we have

$$
f(u)=x(\sigma(u))=\sigma^{*}(x)(u)=x^{q}(u)=u^{q} .
$$

Hence $\left|U_{\sigma}\right|=q$, as claimed.

We have now shown that a long root subgroup of $H_{\sigma}$ has order $q$, from which the conclusion follows.

Finally, assume that $G_{\sigma}$ is a Suzuki or Ree group. Then $\sigma^{2}$ is a $q$-power field morphism of $G$, and from the above proof we have $\left|U_{\sigma^{2}}\right|=q$, where $U$ is a $\sigma^{2}$-stable long root subgroup of $H$. As $\sigma$ squares to $\sigma^{2}$, it follows that either $\sigma$ induces a field morphism of $H$, or $H_{\sigma}$ is a Suzuki or Ree group. In the latter case we have the result. The former leads to a contradiction, as here we can choose $U$ to be $\sigma$-stable and obtain $\left|U_{\sigma}\right|=q^{1 / 2}$. But this is impossible as $q$ is an odd power of $p$.

We conclude this section by stating a result concerning the centralizers of semisimple elements of prime order in simple algebraic groups; this result is taken from [GL, 14.1].

Proposition 1.14. Let $G$ be a simple algebraic group, and let $\alpha_{1}, \ldots, \alpha_{l}$ be a system of fundamental roots for the root system of $G$; let $\alpha_{0}=\sum c_{i} \alpha_{i}$ be the highest root. Suppose $x \in G$ is a semisimple element of prime order $r$. Then $C_{G}(x)^{0}$ is a 
reductive subgroup of maximal rank in $G$, and if $\Delta$ is the Dynkin diagram of the root system of $\left(C_{G}(x)^{0}\right)^{\prime}$, then one of the following holds:

(i) $\Delta$ is obtained by deleting nodes from the Dynkin diagram of $G$;

(ii) $\Delta$ is obtained from the extended Dynkin diagram of $G$ by deleting one node $\alpha_{i}$, where $r=c_{i}$.

\section{Proof of Proposition 2 and Corollary 3}

The proof of Proposition 2 relies upon the following result.

Proposition 2.1. Let $G$ be a simple algebraic group over the algebraically closed field $K$, let $T$ be a maximal torus of $G$ and let $X(T)$ be the character group of $T$. For any subgroup $L$ of $X(T)$, define

$$
\operatorname{Ann}(L)=\{t \in T: \lambda(t)=1 \text { for all } \lambda \in L\}
$$

Then the exponent of $\operatorname{Ann}(L) /(\operatorname{Ann}(L))^{0}$ divides $t(L)$ (the exponent of the torsion subgroup of $X(T) / L)$.

Proof. Choose a $\mathbb{Z}$-basis $f_{1}, \ldots, f_{n}$ of $X(T)$ such that $n_{1} f_{1}, \ldots, n_{r} f_{r}$ is a $\mathbb{Z}$-basis of $L$, for some positive integers $n_{1}, \ldots, n_{r}$. Define a morphism $h: T \rightarrow\left(K^{*}\right)^{n}$ by

$$
h(t)=\left(f_{1}(t), \ldots, f_{n}(t)\right) \quad \text { for } t \in T .
$$

Then $h$ has comorphism $h^{*}$ such that $h^{*}\left(\pi_{i}\right)=f_{i}$ (where $\pi_{i}$ is the $i$ th projection map on $\left.\left(K^{*}\right)^{n}\right)$. It follows that $h^{*}$ is an isomorphism. The surjectivity of $h^{*}$ implies that $h$ is injective. Since the image of $h$ is closed, dimension considerations imply that $h$ is an isomorphism of algebraic groups. Thus, if

$$
\begin{gathered}
T_{1}=h^{-1}\left\{\left(\alpha_{1}, \ldots, \alpha_{r}, 1, \ldots, 1\right): \alpha_{i} \in K^{*}\right\}, \\
T_{2}=h^{-1}\left\{\left(1, \ldots, 1, \alpha_{r+1}, \ldots, \alpha_{n}\right): \alpha_{i} \in K^{*}\right\},
\end{gathered}
$$

then $T=T_{1} \times T_{2}$, and both $T_{1}$ and $T_{2}$ are tori. Also, $X(T)=X\left(T_{1}\right) \times X\left(T_{2}\right)$ and $L \leq X\left(T_{1}\right)$.

Write $g_{i}=n_{i} f_{i}$ for $1 \leq i \leq r$, and define $d: T \rightarrow\left(K^{*}\right)^{n}$ by

$$
d(t)=\left(g_{1}(t), \ldots, g_{r}(t), 1, \ldots, 1\right) \quad \text { for } t \in T .
$$

Then $\operatorname{Ann}(L)=\operatorname{ker} d$, and $(\operatorname{ker} d)^{0}=T_{2}$. Let $t \in \operatorname{Ann}(L)$ and $e=\exp \left(X\left(T_{1}\right) / L\right)$. As $X\left(T_{1}\right) / L$ is the torsion group of $X(T) / L$, we have $e=t(L)$. We need to show that $t^{e} \in T_{2}$. This is equivalent to $\chi\left(t^{e}\right)=e \chi(t)=1$ for all $\chi \in X\left(T_{1}\right)$. However, if $\chi \in X\left(T_{1}\right)$, then $e \chi \in L$, so that $e \chi(t)=1$, as required.

Proof of Proposition 2. As in the hypothesis of Proposition 2, let $G$ be a simple adjoint algebraic group, and let $x$ be a semisimple element of $G$ of finite order greater than $m t(\Sigma(G))$, for some positive integer $m$. We aim to find an infinite closed subgroup $S$ of $G$ satisfying conclusions (i) and (ii) of Proposition 2.

Choose a maximal torus $T$ of $G$ containing $x$, and let $X(T)$ denote the character group of $T$. Regard the root system $\Sigma(G)$ of $G$ as a subset of $X(T)$ in the usual way. As $G$ is adjoint, $\mathbb{Z} \Sigma=X(T)$. Define subgroups $L, L^{\prime}$ of $\mathbb{Z} \Sigma$ as follows:

$$
\begin{gathered}
L^{\prime}=\langle\alpha-\beta: \alpha, \beta \in \Sigma(G) \text { and } \alpha(x)=\beta(x)\rangle, \\
L=\langle\alpha-\beta, \gamma: \alpha, \beta, \gamma \in \Sigma(G), \alpha(x)=\beta(x) \text { and } \gamma(x)=1\rangle .
\end{gathered}
$$


Since $\gamma(x)=1$ implies that $\gamma(x)=\gamma(x)^{-1}$, hence $2 \gamma \in L^{\prime}$, we see that $t(L)$ (the exponent of the torsion group of $\mathbb{Z} \Sigma / L)$ divides $t\left(L^{\prime}\right)$. By the definition of $t(\Sigma(G))$, we have $t\left(L^{\prime}\right) \leq t(\Sigma(G))$, and so $t(L) \leq t(\Sigma(G))$.

Let $S=\operatorname{Ann}(L)$, the annihilator of $L$ in $T$. Clearly $x \in S$. By 2.1, the exponent of $S / S^{0}$ divides $t(L)$. Since $x$ has order greater than $m t(\Sigma(G))$, this means that some nontrivial power of $x$, of order greater than $m$, lies in $S^{0}$. In particular $S^{0} \neq 1$. Thus conclusion (i) of Proposition 2 holds.

To prove (ii) of Proposition 2, write

$$
L(G)=L_{0} \oplus \sum_{\alpha \in \Sigma(G)} L_{\alpha},
$$

where $L_{0}=L(T)$, and for $\alpha \in \Sigma(G)$,

$$
L_{\alpha}=\{v \in L(G): t(v)=\alpha(t) v \text { for all } t \in T\} .
$$

Let $W$ be an $x$-invariant subspace of $L(G)$. For a character $\chi$ of $\langle x\rangle$, set $W_{\chi}=$ $\{v \in W: x(v)=\chi(x) v\} ;$ then

$$
W=\sum_{\chi} W_{\chi}
$$

the sum being over all irreducible characters $\chi$ of $\langle x\rangle$. For nontrivial such $\chi$, we have

$$
W_{\chi} \subseteq L(\chi)=\sum\left\{L_{\alpha}: \alpha \in \Sigma(G), \alpha(x)=\chi(x)\right\} .
$$

If $\alpha_{1}, \ldots, \alpha_{k}$ are the roots such that $\alpha_{i}(x)=\chi(x)$, then $\alpha_{i}-\alpha_{j} \in L$ for $i \neq j$; hence $\alpha_{i}(s)=\alpha_{j}(s)$ for all $s \in S$. It follows that each $s \in S$ acts on $L(\chi)$ as a scalar multiple of the identity, and hence $S$ fixes $W_{\chi}$. Finally, to see that $S$ fixes $W_{1}=C_{W}(x)$, observe that

$$
W_{1} \subseteq L_{0}+L(1)=L_{0}+\sum\left\{L_{\alpha}: \alpha \in \Sigma(G), \alpha(x)=1\right\} .
$$

If $\alpha(x)=1$ then $\alpha \in L$, hence $\alpha(s)=1$ for all $s \in S$; thus $S$ acts as the identity on $L_{0}+L(1)$, so $S$ fixes $W_{1}$. We conclude that $S$ stabilizes $W$, completing the proof of Proposition 2.

Proof of Corollary 3. Assume that $G$ is a simple adjoint algebraic group in characteristic $p$, and that $X=X(q)<G$, with $X$ quasisimple of Lie type over $\mathbb{F}_{q}$ $\left(q=p^{e}\right)$. Assume also that $q>t(\Sigma(G)) m(X)$, where $m(X)$ is the order of the Schur multiplier of $X / Z(X)$.

Observe that $X$ contains a subgroup isomorphic to $S L_{2}(q)$ or ${ }^{2} B_{2}(q)$, unless $X=L_{2}(q)$ or ${ }^{2} G_{2}(q)$. Excluding the latter possibilities for $X$, we see that $X$ has a semisimple element $x$ of order at least $q+1$, and by assumption this is greater than $t(\Sigma(G)) \cdot|Z(X)|$; and when $X=L_{2}(q)$ or ${ }^{2} G_{2}(q), X$ has an element of order $(q+1) /(2, p-1)$ or $q+\sqrt{3 q}+1$ respectively, which is again greater than $t(\Sigma(G)) \cdot|Z(X)|$. Applying Proposition 2 with $m=|Z(X)|$, we see that there is an infinite closed subgroup $S$ of $G$ such that $x \in S, 1 \neq x^{n} \in S^{0}-Z(X)$ for some $n$, and every $x$-invariant subspace of $L(G)$ is $S$-invariant. Define

$$
\bar{X}=\langle X, S\rangle^{0} \text {. }
$$

Then $x^{n} \in(X \cap \bar{X})-Z(X)$; hence, as $X \cap \bar{X}$ is normal in $X$ and $X$ is quasisimple, we have $X<\bar{X}$. Also every $X$-invariant subspace of $L(G)$ is certainly $x$-invariant, 
hence is also $\langle X, S\rangle$-invariant, and therefore is $\bar{X}$-invariant. This completes the proof of part (i) of Corollary 3.

For part (ii) of Corollary 3, suppose that $X \leq G_{\sigma}$, where $\sigma$ is a Frobenius morphism. Let $\mathcal{M}$ be the collection of all $X$-invariant subspaces of all $G$-composition factors of $L(G)$, and define

$$
Y=\bigcap_{W \in \mathcal{M}} G_{W}
$$

By 1.12(iii), $Y$ is $\sigma$-stable; also $Y$ is clearly closed and contains the subgroup $\bar{X}$ defined above. Clearly $Y$ fixes every $X$-invariant subspace of each $G$-composition factor of $L(G)$, so this completes the proof of part (ii) of Corollary 3 .

\section{Proof of Theorem 1: Strategy and lemmas}

We embark upon the proof of Theorem 1. In this section, after some preliminary observations, we explain the strategy of our proof, which is based on the consideration of the centralizer of a suitably chosen element $a$ of the subgroup $X$. We then present some general lemmas concerning this centralizer.

Assume that $G$ is a simple adjoint algebraic group of exceptional type over the algebraically closed field $K$ of characteristic $p$, and that $X=X(q)$ is a quasisimple group of Lie type over $\mathbb{F}_{q}\left(q=p^{e}\right)$, with $X<G$. The case where $X=A_{1}(q),{ }^{2} B_{2}(q)$ or ${ }^{2} G_{2}(q)$ follows from Corollary 3 , so we assume that $X$ is not one of these groups. Suppose further that $q>9$ and $X \neq A_{2}^{\epsilon}(16)$ (as in the hypothesis of Theorem $1)$. We aim to show that $X$ lies in a connected subgroup $\bar{X}$ of $G$ fixing the same subspaces of $L(G)$ as $X$. This is enough to complete the proof of Theorem 1, since part (ii) of Theorem 1 follows exactly as at the end of $\S 2$.

We begin with a lemma which restricts attention to the case where $G=E_{8}$.

Lemma 3.1. If Theorem 1 holds for $G=E_{8}$, then it holds in all cases.

Proof. Suppose that Theorem 1 holds for $G=E_{8}$. We deduce the result for $G=E_{7}$ as follows. Suppose $X=X(q)<E_{7}$, with $q$ as in the hypothesis of Theorem 1 . By the conclusion of Theorem 1 for $E_{8}$, there is a connected subgroup $\bar{X}$ of $E_{8}$ containing $X$ which fixes exactly the same subspaces of $L\left(E_{8}\right)$ as $X$ does. Since $X$ fixes $L\left(E_{7}\right)$, so does $\bar{X}$, and therefore $\bar{X}$ lies in $N_{E_{8}}\left(L\left(E_{7}\right)\right)$; this is equal to $A_{1} E_{7}$ (since it certainly contains $A_{1} E_{7}$, which is maximal in $E_{8}$ ). Passing to the actions of $X, \bar{X}$ on $L\left(E_{7}\right)$, and projecting to the adjoint group $E_{7}$, we have the conclusion of Theorem 1 for $E_{7}$. Theorem 1 for $G=E_{6}, F_{4}, G_{2}$ follows using the same argument.

In view of Lemma 3.1, we assume from now on that $G=E_{8}$.

Observe that $X$ contains a subgroup $A$ isomorphic to $A_{2}^{\epsilon}(q)(\epsilon= \pm)$ or $B_{2}(q)$. There is an element $a \in A$ such that $C_{A}(a)^{(\infty)}=S \cong S L_{2}(q)$ generated by long root subgroups of $A$, and such that $a$ has order divisible by $(q-\epsilon) /(3, q-\epsilon)$ if $A \cong A_{2}^{\epsilon}(q)$, and divisible by $q+1$ if $A \cong B_{2}(q)$. By [SS, II, 4.4], $C_{G}(a)$ is a connected reductive group; write

$$
D=C_{G}(a)^{\prime},
$$

so that $D$ is connected and semisimple, and $S<D$. Note that $o(a) \geq 6$, except when $X=L_{3}(13)$ or $U_{3}(11)$.

The strategy of our proof is based on the following elementary lemma. 
Lemma 3.2. Suppose that there is a subgroup $Y$ of $X$ and a closed connected subgroup $Z$ of $G$ such that

(i) $X \cap Z \not \leq Z(X)$, and

(ii) every $Y$-invariant subspace of $L(G)$ is $Z$-invariant. Then the conclusion of Theorem 1 holds.

Proof. Take $\bar{X}=\langle X, Z\rangle^{0}$. Then $X \cap \bar{X}$ contains $X \cap Z$, so $X \cap \bar{X} \not \leq Z(X)$, and hence $X<\bar{X}$. By (ii), $X$ and $\bar{X}$ fix the same subspaces of $L(G)$.

In the ensuing proof, we attempt to fulfil the hypotheses of 3.2 using two basic methods. The first method is to try to find a torus $T_{k}$ such that $\langle a\rangle \cap T_{k}$ and $T_{k}$ fix the same subspaces of $L(G)$. Then, assuming $\langle a\rangle \cap T_{k} \not \leq Z(X)$, the hypotheses of 3.2 hold, taking $Y=\langle a\rangle \cap T_{k}, Z=T_{k}$.

This method only works in relatively few cases (usually when $D=C_{G}(a)^{\prime}$ has large rank). In other cases, we use the following approach. Observe that $S<D$, and with a couple of exceptions, $D$ is a product of classical groups. By studying the possible embeddings of $S$ in $D$, we attempt to find a closed subgroup $\bar{S} \cong S L_{2}(K)$ of $D$ containing $S$, such that every composition factor of $\bar{S}$ on $L(G)$ has high weight less than $q$. Then by $1.5, S$ and $\bar{S}$ fix the same subspaces of $L(G)$, so the hypotheses of 3.2 hold with $Y=S, Z=\bar{S}$. It turns out (after much work) that this approach is successful in most cases; the remaining cases are usually dealt with using the representation theory of the subgroup $A$.

It is convenient to carry out the above strategy by contradiction; thus, in view of 3.2 , we assume

there are no subgroups $Y \leq X, Z \leq G$ such that $Z$ is closed and connected, $X \widehat{\cap} Z \not \leq Z(X)$ and every $Y$ invariant subspace of $L(G)$ is $Z$-invariant.

We consider now the possibilities for $C_{G}(a)$. Recall that the extended Dynkin diagram of $G=E_{8}$ is

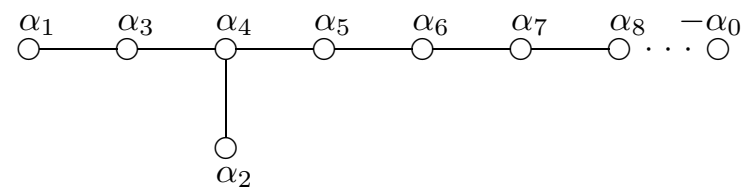

and the highest root $\alpha_{0}=2 \alpha_{1}+3 \alpha_{2}+4 \alpha_{3}+6 \alpha_{4}+5 \alpha_{5}+4 \alpha_{6}+3 \alpha_{7}+2 \alpha_{8}$.

Lemma 3.3. Suppose that $o(a) \geq 6$. Then one of the following holds:

(i) $C_{G}(a)=T_{k} D$, where $T_{k}$ is a central torus of rank $k \geq 1$, and $D$ is a connected semisimple group;

(ii) $C_{G}(a)=A_{1} A_{2} A_{5}$ and $o(a)=6$.

Proof. This follows easily from 1.14: if (i) does not hold, then every power of $a$ of prime order $r$ has centralizer obtained by deleting a node from the extended Dynkin diagram of $G$ corresponding to a coefficient $r$ in the above expression for $\alpha_{0}$. Repeating this process, we find that the only possibility with $o(a) \geq 6$ is that in conclusion (ii). 
Lemma 3.4. Suppose that $o(a) \geq 6$ and $C_{G}(a)=T_{1} D$, with $T_{1}$ a rank 1 torus and $D$ semisimple. Then $C_{G}\left(T_{1}\right)=L_{i}$, a Levi subgroup corresponding to the Dynkin diagram of $G$ with the node $\alpha_{i}$ deleted. Moreover $D \leq L_{i}$, and one of the possibilities in Table 1 holds.

TABLE 1

\begin{tabular}{llll}
$L_{i}$ & $D$ & $o(a)$ & possible $q$ \\
\hline$D_{7}$ & $A_{1} A_{1} D_{5}$ or $A_{3} D_{4}$ & $o\left(a^{2}\right) \leq 4$ & $17,19,23,25$ \\
$A_{7}$ & $A_{7}$ & $o(a) \leq 6$ & 17,19 \\
$A_{1} A_{6}$ & $A_{1} A_{6}$ & $o(a) \leq 8$ & \\
$A_{1} A_{2} A_{4}$ & $A_{1} A_{2} A_{4}$ & $o(a) \leq 12$ & \\
$A_{3} A_{4}$ & $A_{3} A_{4}$ & $o(a) \leq 10$ & \\
$A_{2} D_{5}$ & $A_{2} D_{5}$ & $o(a) \leq 8$ & $17,19,23,25$ \\
& $A_{2} A_{1} A_{1} A_{3}$ & $o\left(a^{2}\right) \leq 8$ & \\
$A_{1} E_{6}$ & $A_{1} E_{6}$ & $o(a) \leq 6$ & 17,19 \\
& $A_{1} A_{1} A_{5}$ & $o\left(a^{2}\right) \leq 6$ & \\
& $A_{1} A_{2} A_{2} A_{2}$ & $o\left(a^{3}\right) \leq 6$ \\
$E_{7}$ & $A_{1} D_{6}$ or $A_{7}$ & $o\left(a^{2}\right) \leq 4$ & $17,19,23,25$ \\
& $A_{1} A_{3} A_{3}$ & $o\left(a^{4}\right) \leq 4$ \\
& $A_{2} A_{5}$ & $o\left(a^{3}\right) \leq 4$ & \\
\hline
\end{tabular}

Proof. Observe that $C_{G}\left(T_{1}\right)$ is a Levi subgroup of $G$ containing $D$, a semisimple group of rank 7; hence $C_{G}\left(T_{1}\right)=L_{i}$ for some $i$.

First suppose that $i=8$, so $L_{i}=E_{7}$. Since the $\alpha_{8}$-coefficient in the highest root $\alpha_{0}$ is 2 , the torus $T_{1}$ has just 5 distinct weights $2,1,0,-1,-2$ on $L(G)$; indeed, this can be seen by writing a typical element $T_{1}(c)$ of $T_{1}$ as

$$
h_{\alpha_{1}}\left(c^{2}\right) h_{\alpha_{2}}\left(c^{3}\right) h_{\alpha_{3}}\left(c^{4}\right) h_{\alpha_{4}}\left(c^{6}\right) h_{\alpha_{5}}\left(c^{5}\right) h_{\alpha_{6}}\left(c^{4}\right) h_{\alpha_{7}}\left(c^{3}\right) h_{\alpha_{8}}\left(c^{2}\right) .
$$

If $a \in T_{1}$, then since $o(a) \geq 6$, every $a$-invariant subspace of $L(G)$ is also $T_{1}$ invariant; hence the subgroups $\langle a\rangle$ of $X$ and $T_{1}$ of $G$ satisfy (i) and (ii) of 3.2, contrary to our assumption ( $\dagger$ ). Therefore $a \notin T_{1}$, and so $a=a_{1} a_{2}$ with $a_{1} \in T_{1}$ and $1 \neq a_{2} \in L_{8}=E_{7}$ and $a_{2} \notin Z\left(E_{7}\right)$. We have $C_{E_{7}}\left(a_{2}\right)=D$, a proper semisimple subgroup of $E_{7}$ of rank 7 . Using 1.14 again, we see that the only possibilities for $D$ are $A_{1} D_{6}, A_{7}, A_{1} A_{3} A_{3}, A_{2} A_{5}$, and $a_{2}^{r} \in Z\left(E_{7}\right)$ with $r=2,2,4,3$, respectively. Then $a^{r} \in T_{1}$; replacing $a$ by $a^{r}$ and using the previous argument, we conclude that $o\left(a^{r}\right)<5$. This completes the proof for $i=8$, and the other values of $i$ are handled in the same way.

Lemma 3.5. Assuming $o(a) \geq 6$, we have $S L_{2}(q) \cong S \leq C_{X}(a)^{\prime}<C_{G}(a)^{\prime}=D$, and one of the following holds:

(1) $D=A_{1} A_{2} A_{5}, o(a)=6$ and $q=17$ or 19 ;

(2) $D$ is as in row $1,2,6,8$ or 11 of Table 1;

(3) $D=D_{6}=L_{18}$;

(4) $D=E_{6}=L_{78}$ and $p=2$; 
(5) $D$ is contained in one of the following subgroups of $G$ :

$$
\begin{array}{ll}
A_{1} A_{3} A_{3}, A_{2} A_{5} & \left(\subseteq L_{8}=E_{7}\right) \\
A_{1} A_{1} A_{5}, A_{1} A_{2} A_{2} A_{2} & \left(\subseteq L_{7}=A_{1} E_{6}\right) \\
A_{2} A_{1} A_{1} A_{3} & \left(\subseteq L_{6}=A_{2} D_{5}\right) \\
A_{1} A_{1} D_{4} & \left(\subseteq L_{18}=D_{6}\right) \\
A_{3} A_{4}, A_{1} A_{2} A_{4}, A_{1} A_{6} & \left(=L_{5}, L_{4}, L_{3} \text { resp. }\right) \\
A_{2} D_{4}, A_{1} D_{5} & \left(=L_{16}, L_{17} \text { resp. }\right) .
\end{array}
$$

Proof. By 3.3, either (1) holds or $C_{G}(a)=T_{k} D$ with $k \geq 1$, so assume the latter holds. When $k=1$, the possibilities are as listed in Table 1 , and visibly either (2) or (5) holds.

Now assume that $k=2$, so $D \leq C_{G}\left(T_{2}\right)=L_{i j}$ for some $i, j$. If $i$ or $j$ is in $\{3,4,5\}$, or if $\{i, j\}$ is $\{1,6\}$ or $\{1,7\}$, then (5) holds. Also $L_{12}, L_{26}, L_{27}, L_{28}$, $L_{67}$ and $L_{68}$ lie in conjugates of subgroups listed in (5) of $L_{3}, L_{4}, L_{7}, L_{3}, L_{17}$ and $L_{17}$, respectively. And if $\{i, j\}=\{1,8\}$ or $\{7,8\}$ then $L_{i j}=D_{6}$ or $E_{6}$, and either $D=L_{i j}$ or $D$ lies in $C_{L_{i j}}(a)$, a proper semisimple subgroup of $L_{i j}$ of rank 6 ; the only possibilities for the latter are $A_{1} A_{1} D_{4}, A_{3} A_{3}$ (if $L_{i j}=D_{6}$ ), or $A_{1} A_{5}, A_{2} A_{2} A_{2}$ (if $L_{i j}=E_{6}$ ), all of which occur under (5). If $D=L_{18}$ then (3) holds. Finally, if $D=L_{78}=E_{6}$, then $p=2$, as in (4): for if $p \neq 2$ then $Z(S)=\langle t\rangle \cong Z_{2}$, and $D \leq C_{L_{78}}(t)$, a contradiction.

To complete the proof, observe that if $k \geq 3$, then $D$ lies in a Levi subgroup $L_{i j k}$ of co-rank 3 . We claim that all such Levi subgroups lie in conjugates of subgroups under (5). To see this, observe that as $L_{3}, L_{4}, L_{5}, L_{16}$ and $L_{17}$ are under (5), we may assume that $\{i, j, k\}$ does not contain $3,4,5,\{1,6\}$ or $\{1,7\}$. Hence $i j k=128,267,268,278$ or 678 . One checks that in each case $L_{i j k}$ lies in a subgroup under (5), giving the claim. This completes the proof.

Lemma 3.6. Suppose that $a \in A_{2}=C_{G}\left(E_{6}\right)$. Then $p$ is not 2 or 3 ; in particular, case (4) of Lemma 3.5 does not occur.

Proof. Assume $p=2$ or 3 . Then $q \geq 16$ if $p=2$ and $q \geq 27$ if $p=3$. Hence either $o(a) \geq 26$, or $o(a)=15$ or 17 (with $q=16$ ), or $o(a)=11$ (with $q=32$ ), or $o(a)=21$ (with $q=64$ ). Choose a torus $T_{2}<A_{2}$ containing $a$. We may take $T_{2}$ to consist of diagonal $3 \times 3$ matrices, and write $a=\operatorname{diag}\left(\alpha, \beta, \alpha^{-1} \beta^{-1}\right)$. The nontrivial composition factors of $A_{2}$ on $L(G)$ are $V_{A_{2}}(10), V_{A_{2}}(01)$ and $V_{A_{2}}(11)$. Hence the eigenvalues of $a$ on $L(G)$ are

$$
1, \alpha, \beta, \alpha \beta, \alpha \beta^{-1}, \alpha^{2} \beta, \alpha \beta^{2}, \alpha^{-1}, \beta^{-1}, \alpha^{-1} \beta^{-1}, \alpha^{-1} \beta, \alpha^{-2} \beta^{-1}, \alpha^{-1} \beta^{-2} .
$$

If these are all distinct, then $T_{2}$ fixes the same subspaces of $L(G)$ as $a$, contrary to assumption $(\dagger)$. Therefore two of the above eigenvalues are equal, and so one of the following holds:

(i) $\alpha^{k}=1, \beta^{k}=1$ or $\alpha^{k}=\beta^{-k}$, for some $k \in\{1,2,3\}$;

(ii) $\alpha^{l}=\beta^{l}, \alpha^{2 l}=\beta^{-l}$ or $\alpha^{-l}=\beta^{2 l}$, for some $l \in\{1,2\}$;

(iii) $\alpha^{2}=\beta, \alpha=\beta^{2}, \alpha^{3}=\beta^{-1}, \alpha^{-1}=\beta^{3}, \alpha^{3}=\beta^{-2}$ or $\alpha^{2}=\beta^{-3}$.

Consider (i). Here $a^{k} \in T_{1}$, where $T_{1}$ is a $W\left(A_{2}\right)$-conjugate of $\left\{\operatorname{diag}\left(1, c, c^{-1}\right)\right.$ : $\left.c \in K^{*}\right\}$. The weights of $T_{1}$ on $L(G)$ are $2,1,0,-1,-2$, so $T_{1}$ and $a^{k}$ fix the same subspaces of $L(G)$ provided $o\left(a^{k}\right) \geq 5$, which is true; this contradicts ( $\dagger$ ) (note that $a^{k} \notin Z(A)$, so $\left.a^{k} \notin Z(X)\right)$. 
In case (ii), $a^{l}$ lies in $T_{1}$, a $W\left(A_{2}\right)$-conjugate of $\left\{\operatorname{diag}\left(c, c, c^{-2}\right): c \in K^{*}\right\}$. Then $T_{1}$ has weights $3,2,1,0,-1,-2,-3$. Hence $T_{1}$ and $a^{l}$ fix the same subspaces provided $o\left(a^{l}\right) \geq 7$, which is true; this again contradicts $(\dagger)$.

Finally in case (iii), $a$ lies in $T_{1}$, a $W\left(A_{2}\right)$-conjugate of $\left\{\operatorname{diag}\left(c, c^{2}, c^{-3}\right): c \in K^{*}\right\}$, which has weights $5,4,3, \ldots,-5$. Then $T_{1}$ and $a$ fix the same subspaces since $o(a) \geq 11$.

Lemma 3.7. Suppose $a \in A_{1} A_{1}=C_{G}\left(D_{6}\right)$. Then either $q$ is prime or $q=25$; in particular, this holds in case (3) of Lemma 3.5. Further, if $q=25$ then $D \neq A_{1} D_{6}$.

Proof. The argument is similar to that of the previous lemma. Assume that $q$ is not prime and that $q \neq 25$. Then either $q \geq 49$ or $q \in\{16,27,32\}$; hence either $o(a) \geq 15$ or $o(a)=11$. We have $a \in T_{2}<A_{1} A_{1}=C_{G}\left(D_{6}\right)$. Write elements of $T_{2}$ as $(c, d)\left(c, d \in K^{*}\right)$, where this represents the element $\left(\operatorname{diag}\left(c, c^{-1}\right), \operatorname{diag}\left(d, d^{-1}\right)\right)$ of $A_{1} A_{1}=S L_{2} \times S L_{2}$. Take $a=(\alpha, \beta)$. Since the nontrivial composition factors of $A_{1} A_{1}$ on $L(G)$ are $1 \otimes 0,0 \otimes 1,2 \otimes 0,0 \otimes 2$ and $1 \otimes 1$, the eigenvalues of $a$ on $L(G)$ are

$$
1, \alpha, \beta, \alpha^{2}, \beta^{2}, \alpha \beta, \alpha \beta^{-1}, \alpha^{-1}, \beta^{-1}, \alpha^{-2}, \beta^{-2}, \alpha^{-1} \beta^{-1}, \alpha^{-1} \beta .
$$

As in the previous lemma, two of these must be equal. Hence one of the following holds:

(i) $\alpha^{k}=1$ or $\beta^{k}=1$ for some $k \in\{1,2,3,4\}$;

(ii) $\alpha=\beta^{ \pm 1}$ or $\alpha^{2}=\beta^{ \pm 2}$;

(iii) $\alpha=\beta^{ \pm 2}$ or $\alpha^{ \pm 2}=\beta$;

(iv) $\alpha=\beta^{ \pm 3}$ or $\alpha^{ \pm 3}=\beta$.

In (ii), $a^{2}$ lies in $T_{1}=\left\{(c, c): c \in K^{*}\right\}$ or $\left\{\left(c, c^{-1}\right): c \in K^{*}\right\}$, both of which have weights $2,1,0,-1,-2$; hence $T_{1}$ and $a^{2}$ fix the same subspaces of $L(G)$ provided $o\left(a^{2}\right) \geq 5$, which is true.

In (iii) or (iv), $a \in T_{1}=\left\{\left(c, c^{m}\right): c \in K^{*}\right\}$ or $\left\{\left(c^{m}, c\right): c \in K^{*}\right\}$, where $m \in\{ \pm 2, \pm 3\}$. If $m= \pm 2$ the weights of $T_{1}$ are $4,3,2, \ldots,-4$, so $T_{1}$ and $a$ fix the same spaces provided $o(a) \geq 9$; this is true by our assumption on $q$. And if $m= \pm 3$, the weights of $T_{1}$ are $\pm 6, \pm 4, \pm 3, \pm 2, \pm 1$; hence $T_{1}$ and $a$ fix the same spaces if $o(a) \geq 13$ or $o(a)=11$, which is again true.

Finally, consider (i). Here $a^{k}$ lies in $T_{1}=\left\{(1, c): c \in K^{*}\right\}$ or $\left\{(c, 1): c \in K^{*}\right\}$, which has weights $2,1,0,-1,-2$; hence $T_{1}$ and $a^{k}$ fix the same subspaces provided $o\left(a^{k}\right) \geq 5$. By our assumption on $q$, this is true unless $k=4$ and $q=49$ (in which case $o(a)$ could be 16). So suppose $k=4$ and $q=49$, and without loss assume that $\alpha^{4}=1$. Recall, that $a=(\alpha, \beta) \in A_{1} A_{1}$. The composition factors of $A_{1} A_{1}$ on $L(G)$ are $0 \otimes 0,1 \otimes 0,0 \otimes 1,2 \otimes 0,0 \otimes 2$ and $1 \otimes 1$. Hence we check that $T_{1}=\left\{(1, c): c \in K^{*}\right\}$ fixes the same spaces as $a$ itself. Finally, if $q=25$ and $D=A_{1} D_{6}$, then $o(a)=8$ by 3.4 , and we may take $a=(\alpha, \pm 1)$; as above, $a$ and $T_{1}=\left\{(1, c): c \in K^{*}\right\}$ fix the same spaces. This completes the proof.

\section{Proof of Theorem 1 for $p \neq 2$ And $q>13$}

We continue with the proof of Theorem 1 . In this section we handle the case where $p \neq 2$ and $q>13$. Recall, from the discussion following Lemma 3.2, that our basic strategy is to try to find a subgroup $\bar{S} \cong S L_{2}(K)$ of $D=C_{G}(a)^{\prime}$ such that $\bar{S}$ contains $S$ and fixes the same subspaces of $L(G)$ as $S$. 
The next lemma provides some useful information for this strategy; it will be used in the following sections also, so we make no assumptions on $p$ and only assume $q>9$ in the statement.

To ease notation, we make the following definition. Suppose $D=E_{1} \ldots E_{k}$, a product of simple factors $E_{i}$, and write $Z=Z(D)$. Denote by $\pi_{i}$ the $i^{\text {th }}$ projection map $D / Z \rightarrow E_{i} Z / Z$. By the projection $S_{i}$ of $S$ in $E_{i}$, we mean a minimal preimage in $E_{i}$ of the group $(S Z / Z) \pi_{i}$. If $E_{i}$ is classical, with usual module $V_{i}$, we abuse notation by writing $V_{i} \downarrow S$ instead of $V_{i} \downarrow \tilde{S}_{i}$, where $\tilde{S}_{i}$ is the derived group of the preimage of $S_{i}$ in a simply connected cover of $E_{i}$. And if each of the $E_{i}$ is classical, we usually describe the embedding of $S$ in $D$ by giving the composition factors of $V_{1} \downarrow S, \ldots, V_{k} \downarrow S$. We can always adjust these factors by applying (globally) any automorphism of $S$ (this amounts to relabelling the elements of $S$ ).

Lemma 4.1. Let $E$ be a simple factor of $D$ of classical type, where $D$ is as in (1), (2), (3) or (5) of Lemma 3.5, and let $V$ be the usual module for E. Suppose that $V \downarrow S$ is not completely reducible. Then one of the following holds:

(i) $p=2$ and $V \downarrow S$ has an indecomposable section $1^{\left(2^{i}\right)} / 0$ or $1^{\left(2^{i}\right)} \otimes 1^{\left(2^{j}\right)} / 1^{\left(2^{j}\right)}$ (with $i \neq j$ );

(ii) $p=3$ and $V \downarrow S$ has an indecomposable section $1^{\left(3^{i}\right)} / 1^{\left(3^{i+1}\right)}, 1^{\left(3^{i}\right)} \otimes 1^{\left(3^{i+1}\right)} / 0$ or $1^{\left(3^{i}\right)} \otimes 1^{\left(3^{i+1}\right)} / 2^{\left(3^{i+1}\right)}$;

(iii) $p=5$ and $V \downarrow S$ has an indecomposable section $3^{\left(5^{i}\right)} / 1^{\left(5^{i+1}\right)}, 3^{\left(5^{i}\right)} \otimes 1^{\left(5^{i+1}\right)} / 0$, $2^{\left(5^{i}\right)} / 1^{\left(5^{i}\right)} \otimes 1^{\left(5^{i+1}\right)}$ or $2^{\left(5^{i}\right)} \otimes 1^{\left(5^{i+1}\right)} / 1^{\left(5^{i}\right)}$;

(iv) $q=p=11$ and $V \downarrow S$ has an indecomposable section $4 / 4,5 / 5$ or $0 / 8 / 0$;

(v) $q=p=13$ and $V \downarrow S$ has an indecomposable section $5 / 5$.

Proof. We have $\operatorname{dim} V \leq 12$; moreover, if $\operatorname{dim} V \geq 8$ then $E=D_{4}, D_{5}, D_{6}$ or $A_{7}$ (with $q$ prime or $q=25$ in the latter two cases). We use Proposition 1.2, which determines the 2-step indecomposables for $S=S L_{2}(q)$.

First assume that $q=p$. By 1.2(i), if there is an indecomposable $W$ for $S$ with composition factors $a / b$, then $a+b=p-3$ or $p-1$, whence $\operatorname{dim} W \geq a+1+b+1 \geq$ $p-1$. As $q>9$, we have $p-1=q-1 \geq 10$. Therefore $E=D_{5}$ or $D_{6}$. Thus $V \downarrow S$ is self-dual, and it follows that either (iv) or (v) holds, or $E=D_{6}, p=11$ and $V \downarrow S$ has an indecomposable section $a / b / a$. The dimension of this section is $2 a+b+3 \leq 12$, so $(a, b)=(0,8)$ or $(1,7)$. The first case is in conclusion (iv). And in the second case, the middle factor (of high weight 7 ) is symplectic, which is impossible.

Now assume $q=p^{e}$ with $e \geq 2$. By 1.2(ii), the following is a list of all 2-step indecomposables for $S$ of dimension at most 10 (of dimension at most 12 if $q=25$ ):

$$
\begin{aligned}
p=2: & 1^{\left(2^{i}\right)} / 0,1^{\left(2^{i}\right)} \otimes 1^{\left(2^{j}\right)} / 1^{\left(2^{j}\right)} \\
p=3: & 1^{\left(3^{i}\right)} / 1^{\left(3^{i+1}\right)}, 1^{\left(3^{i}\right)} \otimes 1^{\left(3^{i+1}\right)} / 0,1^{\left(3^{i}\right)} \otimes 1^{\left(3^{i+1}\right)} / 2^{\left(3^{i+1}\right)}, 1^{\left(3^{i}\right)} \otimes 2^{\left(3^{i+1}\right)} / 1^{\left(3^{i+1}\right)} \\
& 1^{\left(3^{i}\right)} \otimes 1^{\left(3^{j}\right)} / 1^{\left(3^{i+1}\right)} \otimes 1^{\left(3^{j}\right)}, 1^{\left(3^{i}\right)} \otimes 1^{\left(3^{i+1}\right)} \otimes 1^{\left(3^{j}\right)} / 1^{\left(3^{j}\right)} \\
p=5: & 3^{\left(5^{i}\right)} / 1^{\left(5^{i+1}\right)}, 3^{\left(5^{i}\right)} \otimes 1^{\left(5^{i+1}\right)} / 0,3 \otimes 1^{(5)} / 2^{(5)}(q=25), \\
& 2^{\left(5^{i}\right)} / 1^{\left(5^{i}\right)} \otimes 1^{\left(5^{i+1}\right)}, 2^{\left(5^{i}\right)} \otimes 1^{\left(5^{i+1}\right)} / 1^{\left(5^{i}\right)}, 2 \otimes 1^{(5)} / 1 \otimes 2^{(5)}(q=25) \\
p=7: & 5^{\left(7^{i}\right)} / 1^{\left(7^{i+1}\right)}, 4^{\left(7^{i}\right)} / 1^{\left(7^{i}\right)} \otimes 1^{\left(7^{i+1}\right)}, 3^{\left(7^{i}\right)} / 2^{\left(7^{i}\right)} \otimes 1^{\left(7^{i+1}\right)} .
\end{aligned}
$$

If $E=A_{r}$ then $\operatorname{dim} V \leq 8$ (and $q=25$ if $\operatorname{dim} V=8$ ), and the only possible indecomposables appearing in $V \downarrow S$ are among those in conclusions (i)-(iii). Otherwise, $E=D_{r}$ with $4 \leq r \leq 6$ (and $q=25$ if $E=D_{6}$ ); as $V$ is orthogonal, we see using 
1.1 that the last three indecomposables listed for $p=3$, the third, fifth and sixth listed for $p=5$, and all the indecomposables listed for $p=7$ cannot occur in $V \downarrow S$. Hence again one of (i)-(iii) holds.

From now on in this section we shall assume that

$$
p \neq 2 \text { and } q>13 \text {. }
$$

Lemma 4.2. Suppose that $D$ is as in (1), (2), (3) or (5) of Lemma 3.5, but $D \neq$ $A_{1} E_{6}$ (and that $p \neq 2, q>13$ ). Thus $D=E_{1} \ldots E_{k}$, a product of classical simple factors $E_{i}$ with usual module $V_{i}$; let $S_{i}$ be the projection of $S$ in $E_{i}$. Then one of the following holds:

(i) for each $i, V_{i} \downarrow S_{i}$ is completely reducible;

(ii) for some $j, V_{j} \downarrow S_{j}$ is not completely reducible; moreover, there is a subgroup $\bar{S}_{j} \cong A_{1}(K)$ of $E_{j}$ containing $S_{j}$, and the composition factors of $V_{j} \downarrow \bar{S}_{j}$ are as in Table 2 (up to a twist); $1)$;

(iii) $q=27, S<D=A_{5}$ or $A_{6}$ with embedding $1 / 1^{(3)} / 1^{(9)} / 0^{m}$ (where $m=0$ or

(iv) $q=27, S<D=A_{1} D_{5}$ with embedding $1^{(9)}$, $\left(1 \otimes 1^{(3)}\right)^{2} / 0^{2}$;

(v) $q=27, S<D=A_{1} D_{5}$ with embedding $1^{(9)},\left(2^{(3)}\right)^{2} / 1 \otimes 1^{(3)}$.

TABLE 2

\begin{tabular}{lll}
$p$ & $E_{j}$ & $V_{j} \downarrow \bar{S}_{j}$ \\
\hline 5 & $A_{5+\delta}(\delta=0,1)$ & $3 / 1^{(5)} / 0^{\delta}$ \\
& $A_{6+\delta}(\delta=0,1)$ & $2 / 1 \otimes 1^{(5)} / 0^{\delta}$ \\
& $A_{7}(q=25)$ & $2 \otimes 1^{(5)} / 1$ \\
& $D_{5+\delta}(\delta=0,1)$ & $3 \otimes 1^{(5)} / 0^{2+2 \delta}$ \\
& $D_{5+\delta}(\delta=0,1)$ & $2^{2} / 1 \otimes 1^{(5)} / 0^{2 \delta}$ \\
3 & $A_{3+\delta}(\delta=0,1,2,3)$ & $1 / 1^{(3)} / 0^{\delta}$ \\
& $A_{4}$ & $1 \otimes 1^{(3)} / 0$ \\
& $A_{6}$ & $1 \otimes 1^{(3)} / 1^{\left(3^{i}\right)} / 0$ \\
& $D_{4+\delta}(\delta=0,1)$ & $1^{2} /\left(1^{(3)}\right)^{2} / 0^{2 \delta}$ \\
& $D_{4+\delta}(\delta=0,1)$ & $1 \otimes 1^{(3)} / 0^{4+2 \delta}$ \\
& $D_{5}$ & $1 \otimes 1^{(3)} / 1^{\left(3^{i}\right)} / 1^{\left(3^{i}\right)} / 0^{2}$ \\
& $D_{5}$ & $1 \otimes 1^{(3)} / 1^{\left(3^{i}\right)} \otimes 1^{\left(3^{l}\right)} / 0^{2}$ \\
\hline
\end{tabular}

Proof. Suppose (i) does not hold, so that some $V_{j} \downarrow S$ is not completely reducible. Write $E=E_{j}, V=V_{j}$. By 4.1, $p=3$ or 5 and $V \downarrow S$ has a section as in 4.1(ii) or 4.1(iii). Let $T$ be a Cartan subgroup of $S$.

Assume first that $p=5$. Relabelling $S$, we may take it that $V \downarrow S$ has an indecomposable section $3 / 1^{(5)}, 2 \otimes 1^{(5)} / 1,3 \otimes 1^{(5)} / 0$ or $2 / 1 \otimes 1^{(5)}$. In the first case, using 1.1 we see that $E=A_{5}, A_{6}$ or $D_{6}$. Suppose $E=A_{5}$ or $A_{6}$; then $V \downarrow S=3 / 1^{(5)}$ or $\left(3 / 1^{(5)}\right) \oplus 0$. By $1.3(\mathrm{i})$, the restriction map $\operatorname{Ext}_{A_{1}(K)}^{1}\left(3,1^{(5)}\right) \rightarrow$ $\operatorname{Ext}_{A_{1}(q)}^{1}\left(3,1^{(5)}\right)$ is an isomorphism. It follows that there is a closed subgroup $A_{1}(K)$ of $E$ containing $S_{j}$, as required for (ii). Now suppose $E=D_{6}$; then $D=E, q=25$ by 3.7 , and $V \downarrow S=1^{(5)} / 3 / 3 / 1^{(5)}$. Pick an element $t \in S$ of order 26 ; then $t$ lies in a Levi subgroup $A_{5}$ of $E$, and we may take it that $t$ lies in a 1-dimensional torus $T_{1}=\left\{\operatorname{diag}\left(c^{5}, c^{-5}, c^{3}, c^{-3}, c, c^{-1}\right): c \in K^{*}\right\}<A_{5}$. As the nontrivial composition 
factors of the restriction of $L(G) / L\left(A_{5}\right)$ to $A_{5}$ are just $V_{A_{5}}\left(\lambda_{i}\right)(1 \leq i \leq 5)$ (see [LS2, §2]), it follows that $t$ and $T_{1}$ fix the same subspaces of $L(G)$, contrary to (†).

Next, if $V \downarrow S$ has an indecomposable section $2 \otimes 1^{(5)} / 1$, then $E=A_{7}$ (use 1.1 again), and we obtain (ii) using 1.3(i), as above.

Now assume $V \downarrow S$ has an indecomposable section $3 \otimes 1^{(5)} / 0$. Then $E=D_{5}$ or $D_{6}$. In the first case, $V \downarrow S$ is an indecomposable module $0 / 3 \otimes 1^{(5)} / 0$, and $S_{j}$ (the projection of $S$ in $E$ ) fixes a singular 1-space of $V$. The stabilizer in $E$ of this 1-space is a parabolic subgroup $Q L$ with unipotent radical $Q$ and Levi subgroup $L=D_{4} T_{1}$, and $Q$ is a $K L$-module of high weight $\lambda_{1}$. If we choose a subgroup $\tilde{S} \cong A_{1}(K)$ of $L$ such that $Q \downarrow \tilde{S}=3 \otimes 1^{(5)}$, then $S_{j}<Q \tilde{S}$. Moreover, by 1.3, $H^{1}(S, Q) \cong H^{1}(\tilde{S}, Q)$, and clearly $C_{Q}(T)=0$. Therefore by $1.6, S_{j}$ lies in a closed complement to $Q$ in $Q \tilde{S}$, giving (ii). When $E=D_{6}$ we have $V \downarrow S=0^{2} / 3 \otimes 1^{(5)} / 0^{2}$. Since $H^{1}\left(S, 3 \otimes 1^{(5)}\right)$ has dimension 1 by 1.2 (ii), in fact $V \downarrow S=V_{2} \perp\left(0 / 3 \otimes 1^{(5)} / 0\right)$, where $V_{2}$ is a non-degenerate 2 -space, so $S_{j}$ lies in a subgroup $D_{5}$ of $E$, and now the argument given for $E=D_{5}$ applies.

To complete the case $p=5$, suppose $V \downarrow S$ has an indecomposable section $2 / 1 \otimes 1^{(5)}$. Then $E=A_{6}, A_{7}, D_{5}$ or $D_{6}$. If $E=A_{6}$ or $A_{7}$, we obtain (ii) using 1.3(i). Now suppose $E=D_{5}$. Then $V \downarrow S$ is an indecomposable module $2 / 1 \otimes 1^{(5)} / 2$. The group $A_{1}(K)$ has a 10-dimensional orthogonal module $\bigwedge^{2} V(4)$, and $\bigwedge^{2} V(4) \downarrow A_{1}(K)=2 / 1 \otimes 1^{(5)} / 2$; moreover this module is indecomposable, since a 1-dimensional unipotent subgroup centralizes only a 1-space of weight 2 vectors in $\bigwedge^{2} V(4)$. Hence $E$ contains a subgroup $\tilde{S} \cong A_{1}(K)$ with this representation on the usual module. We may take it that both $S_{j}$ and $\tilde{S}$ lie in the same parabolic subgroup $Q L$ stabilizing a totally singular 3-space. The unipotent radical $Q$ has an $\tilde{S}$-series $1<Q_{1}<Q$ with $Q_{1}, Q / Q_{1}$ abelian, such that $Q_{1} \downarrow \tilde{S}=V(2)$ and $\left(Q / Q_{1}\right) \downarrow \tilde{S}=2 \otimes 1 \otimes 1^{(5)}=\left(3 \otimes 1^{(5)}\right) \oplus\left(1 \otimes 1^{(5)}\right)$. By $1.2, H^{1}\left(\tilde{S}, Q / Q_{1}\right)$ is 1-dimensional. Hence, under the action of $\left(Q / Q_{1}\right) Z(L)$ there are just two classes of closed complements to $Q / Q_{1}$ in $\left(Q / Q_{1}\right) \tilde{S}$, with representatives $\tilde{S}$ and $\bar{S}$, where $\bar{S}=L \cap Q \tilde{S}$. As $H^{1}\left(\tilde{S}, Q / Q_{1}\right) \cong H^{1}\left(S, Q / Q_{1}\right)$ by 1.3 , and $C_{Q / Q_{1}}(T)=0$, we deduce from 1.6 that $S_{j}$ therefore lies in a conjugate of either $Q_{1} \tilde{S}$ or $Q_{1} \bar{S}$. Since also by $1.3, H^{1}\left(\tilde{S}, Q_{1}\right) \cong H^{1}\left(S, Q_{1}\right)=0, S$ lies in a conjugate of $\tilde{S}$ or $\bar{S}$, giving the conclusion.

Finally, suppose $E=D_{6}$. If $S_{j}$ lies in a subgroup $D_{5}$ of $E$, then the conclusion follows as before. Otherwise, $V \downarrow S=\left(1 \otimes 1^{(5)} / 2 / 1 \otimes 1^{(5)}\right) \oplus 0$; also $q=25$ and $D=E$, by 3.7. An element $t$ of $S$ of order 26 lies in a 1-dimensional torus

$$
T_{1}=\left\{\operatorname{diag}\left(c^{6}, c^{4}, c^{-4}, c^{-6}, c^{2}, 1, c^{-2}, c^{6}, c^{4}, c^{-4}, c^{-6}, 1\right): c \in K^{*}\right\}<D_{6}
$$

(matrices relative to a suitable basis). The nontrivial composition factors of $L(G) \downarrow E$ are $V_{D_{6}}\left(\lambda_{i}\right)(i=1,2,5,6)$. Moreover, $T_{1}$ lies in a Levi subgroup $A_{4}$ of $D_{6}$, which by [LS2, 2.6] has all its nontrivial composition factors on $V_{D_{6}}\left(\lambda_{i}\right)(i=5,6)$ among the modules $V_{A_{4}}\left(\lambda_{1}\right), \ldots, V_{A_{4}}\left(\lambda_{4}\right)$. Hence the eigenvalues on $L(G)$ of a typical element $T_{1}(c)$ of $T_{1}$ displayed above are among $c^{ \pm k}$ with $0 \leq k \leq 12$. It follows that $T_{1}$ and $t$ fix the same subspaces of $L(G)$, contrary to $(\dagger)$. This completes the proof for $p=5$.

Now suppose that $p=3$. By $4.1, V \downarrow S$ has an indecomposable section $1 \otimes 1^{(3)} / 0$, $1 / 1^{(3)}$ or $1 \otimes 1^{(3)} / 2^{(3)}$. Consider the first case. Here $E=A_{r}, D_{4}$ or $D_{5}$ (note that $E \neq D_{6}$ by 3.7). Suppose $E=A_{r}$. Then $r \neq 7$ by 3.4, and $r=4$ yields to the 
usual argument using 1.3(i). If $r=5$ then the projection $S_{j}$ of $S$ in $E$ is $P S L_{2}(q)$, so $D=A_{1} A_{5}$; but then $a \in C_{G}\left(A_{1} A_{5}\right)=A_{2}=C_{G}\left(E_{6}\right)$, contrary to 3.6. The same argument works if $r=6$, unless $V \downarrow S=\left(1 \otimes 1^{(3)} / 0\right) \oplus 1^{3^{(i)}}$; but then $S<A_{1} A_{4}<A_{6}$ and we can apply the 1.3(i) argument again.

When $E=D_{4}$ or $D_{5}$, one of the following holds:

(a) $V \downarrow S=V_{m} \perp\left(0 / 1 \otimes 1^{(3)} / 0\right)$, with $V_{m}(m=2$ or 4$)$ a non-degenerate $m$-space on which $S$ acts completely reducibly;

(b) $E=D_{5}$ and $V \downarrow S=0 / 1 \otimes 1^{(3)} / 1^{\left(3^{i}\right)} \otimes 1^{\left(3^{i+1}\right)} / 0$ (with $S$ fixing a singular 1-space);

(c) $E=D_{5}$ and $V \downarrow S=\left(1 \otimes 1^{(3)} / 0 / 1 \otimes 1^{(3)}\right) \perp V_{1}$ (with $S$ fixing a singular 4 -space).

In case (a), $S_{j}$ lies in a subgroup of $E$ of type $S O_{m} \times S O_{6}$, and we can use the usual $\mathrm{H}^{1}$ argument for the $\mathrm{SO}_{6}$ to obtain conclusion (ii) of the lemma. In case (b), observe that $S_{j}$ lies in a subgroup $Q \tilde{S}$ of $E$ fixing a singular 1-space, with $\tilde{S} \cong A_{1}(K)$ and $Q \downarrow \tilde{S}=\left(1 \otimes 1^{(3)}\right) \oplus\left(1^{\left(3^{i}\right)} \otimes 1^{\left(3^{i+1}\right)}\right)$. Now by $1.2, H^{1}(S, Q)$ and $H^{1}(\tilde{S}, Q)$ both have dimension 2 , and $C_{Q}(T)=0$, so conclusion (ii) follows from 1.6. In case (c), the projection $S_{j}$ of $S$ in $E$ is $P S L_{2}(q)$, so $D=A_{1} D_{5}$ (note that $D$ has rank at most 6 by 3.4). Let the projection of $S$ in the factor $A_{1}$ be $1^{\left(3^{k}\right)}$. Pick $t \in S$ of order $q+1$; then $t$ lies in a rank 1 torus $T_{1}$ in $D=A_{1} D_{5}$, with

$$
T_{1}=\left\{\operatorname{diag}\left(c^{3^{k}}, c^{-3^{k}}\right), \operatorname{diag}\left(c^{4}, c^{4}, c^{2}, c^{2}, c^{-4}, c^{-4}, c^{-2}, c^{-2}, 1,1\right): c \in K^{*}\right\}
$$

(matrices in $D_{5}$ relative to a suitable basis). The nontrivial composition factors of $L(G) \downarrow A_{1} D_{5}$ are $0 \otimes \lambda_{i}, 1 \otimes \lambda_{i}(i=1,4,5)$ and $1 \otimes 0$, together with $L\left(A_{1}\right)$ and $L\left(D_{5}\right)$. Hence the eigenvalues of a typical element $T_{1}(c)$ of $T_{1}$ shown above on $L(G)$ are

$$
c^{ \pm 2.3^{k}}, c^{ \pm 3^{k} \pm 6,4,2,0}, c^{ \pm 8,6,4,2,0} .
$$

It follows that $t$ and $T_{1}$ fix the same subspaces of $L(G)$, unless $q=27$ and $3^{k}=9$; this is the exceptional embedding in conclusion (iv) of the lemma.

Now suppose $V \downarrow S$ has an indecomposable section $1 \otimes 1^{(3)} / 2^{(3)}$. Here $E=$ $A_{6}, A_{7}, D_{5}$ or $D_{6}$. In the $A_{6}, A_{7}$ cases, the projection of $S$ in $E$ is $P S L_{2}(q)$, so $D$ has a further factor; but then $D$ has rank at least 7 , which contradicts 3.4; and $E \neq D_{6}$ by 3.7. Therefore $E=D_{5}$; hence $D=D_{5}$ or $A_{1} D_{5}$. We have $V \downarrow S=2^{(3)} / 1 \otimes 1^{(3)} / 2^{(3)}$, indecomposable with $S$ fixing a singular 3 -space. Pick $t \in S$ of order $q+1 \geq 28$. If $S<E$ then $t$ lies in a 1-dimensional torus $T_{1}=$ $\left\{\operatorname{diag}\left(c^{6}, c^{-6}, c^{6}, c^{-6}, 1,1, c^{4}, c^{2}, c^{-2}, c^{-4}\right): c \in K^{*}\right\}$ in a subgroup of type $\mathrm{SO}_{6} \times \mathrm{SO}_{4}$ in $E$. The nontrivial composition factors of $L(G) \downarrow D_{5}$ have high weights $\lambda_{1}, \lambda_{2}, \lambda_{4}$ and $\lambda_{5}$ (see [LS2, §2]). Hence the highest weight of $T_{1}$ on $L(G)$ is 12 . It follows that $t$ and $T_{1}$ fix the same subspaces of $L(G)$, contrary to $(\dagger)$. Hence $S \nless E$, so $D=A_{1} D_{5}$, with embedding $1^{\left(3^{i}\right)}, 2^{(3)} / 1 \otimes 1^{(3)} / 2^{(3)}$. Now an obvious adjustment of the previous $T_{1}$ argument goes through, unless $q=27$ and $3^{i}=9$, as in conclusion (v).

To complete the proof, suppose $V \downarrow S$ has an indecomposable section $1 / 1^{(3)}$. Here $E=A_{r}, D_{4}$ or $D_{5}$. In the $D_{4}, D_{5}$ cases, we have $V \downarrow S=\left(1 / 1^{(3)} / 1^{(3)} / 1\right) \perp V_{k}$, where $k=0$ or 2 and $V_{k}$ is a nondegenerate $k$-space. Thus $S_{j}<D_{4} \leq E$. As $\operatorname{Ext}_{S}^{1}\left(1,1^{(3)}\right)$ has dimension 1 by $1.2, V \downarrow S$ has a submodule $1 \oplus 1^{(3)}$ which is totally singular. Hence $S_{j}$ lies in a subgroup $Q \tilde{S}$ of $E$ stabilizing this singular 4space, where $\tilde{S} \cong A_{1}(K)$ and $Q \downarrow \tilde{S}=\bigwedge^{2}\left(1 \oplus 1^{(3)}\right) \cong\left(1 \otimes 1^{(3)}\right) \oplus 0^{2}$. By 1.2 we have 
$H^{1}(\tilde{S}, Q) \cong H^{1}(S, Q)$; since also $C_{Q}(T)=C_{Q}(\tilde{T})$ (where $\tilde{T}$ is a Cartan subgroup of $\tilde{S})$, the conclusion now follows from 1.6.

Finally, suppose $E=A_{r}$. If $S$ has only two nontrivial composition factors on $V$, then $S_{j}<A_{3} A_{r-4}<E$, and we can use the usual 1.3(i) argument for the $A_{3}$ factor to obtain the conclusion. So assume that $S$ has more than two nontrivial composition factors on $V$. In particular, $r \geq 5$. If $r=7$, or if $D$ has a further factor, 3.4 or 3.6 gives a contradiction. Thus $D=A_{5}$ or $A_{6}$, and $V \downarrow S=1 / 1^{(3)} / 1^{\left(3^{k}\right)} / 0^{m}$, where $m=r-5$. Let $t \in S$ have order $q+1$; then $t$ lies in a rank 1 torus $T_{1}=\left\{\operatorname{diag}\left(c, c^{3}, c^{3^{k}}, c^{-1}, c^{-3}, c^{-3^{k}}, 1^{m}\right): c \in K^{*}\right\}$ of $D$, and we check that $t$ and $T_{1}$ fix the same subspaces of $L(G)$ unless $q=27$ and $3^{k}=9$, as in conclusion (iii) of the lemma.

We now explain how we propose to use Lemma 4.2. The special configurations in $4.2(\mathrm{iii}, \mathrm{iv}, \mathrm{v})$ will be postponed until later (see 4.8). Thus suppose we are in the situation of conclusion (i) or (ii) of 4.2. Consider a module $V_{i}$ such that $V_{i} \downarrow S_{i}$ is completely reducible. Write $V_{i} \downarrow S_{i}=\bigoplus_{r=1}^{t} W_{i_{r}}$, where the $W_{i_{r}}$ are irreducible $S_{i}$-submodules if $E_{i}$ is of type $A_{n}$, and are minimal non-degenerate $S_{i}$-submodules if $E_{i}$ is of type $D_{n}$. Using 1.1, we see that the action of $S_{i}$ on each $W_{i_{r}}$ extends to an action of $A_{1}(K)$, preserving the quadratic form when $E_{i}=D_{n}$. Therefore $S_{i}$ lies in a subgroup $R_{i}=\bar{S}_{i_{1}} \ldots \bar{S}_{i_{t}}$ of $E_{i}$, where each $\bar{S}_{i_{r}} \cong A_{1}(K)$.

Thus if 4.2(i) or (ii) holds, then $S$ lies in a connected subgroup of $D$ which is a commuting product of $A_{1}$ 's and contains each of the $R_{i}$. Abusing notation slightly, write $\bar{S}_{1} \ldots \bar{S}_{l}$ for this commuting product, where each $\bar{S}_{i} \cong A_{1}(K)$. So we have

$$
S<\bar{S}_{1} \ldots \bar{S}_{l} \leq D .
$$

Obviously $\bar{S}_{1} \ldots \bar{S}_{l}$ is an image of $\left(S L_{2}(K)^{l}\right.$. In the ensuing discussion we shall consider subgroups $S L_{2}(K)$ of $\bar{S}_{1} \ldots \bar{S}_{l}$ containing $S$. When $l>1$, there are many such groups, as described below.

Fix matrix groups $S L_{2}(q)<S L_{2}(K)$. For powers $p^{i_{1}}, \ldots, p^{i_{l}}<q$, the embedding $\phi: x \rightarrow\left(x^{\left(p^{i_{1}}\right)}, \ldots, x^{\left(p^{i_{l}}\right)}\right)$ of $S L_{2}(q)$ in $S L_{2}(K)^{l}$ extends to an embedding $\bar{\phi}: \bar{x} \rightarrow\left(\bar{x}^{\left(p^{i_{1}}\right)}, \ldots, \bar{x}^{\left(p^{i_{l}}\right)}\right)$ of $S L_{2}(K)$ in $S L_{2}(K)^{l}$. Every subgroup of $S L_{2}(K)^{l}$ which is isomorphic to $S L_{2}(q)$ and projects nontrivially to each factor is conjugate in $S L_{2}(K)^{l}$ to $\operatorname{Im} \phi$ for some such $\phi$. Notice that it is possible to have such embeddings $\phi, \psi$ such that $\operatorname{Im} \phi=\operatorname{Im} \psi$ but $\operatorname{Im} \bar{\phi} \neq \operatorname{Im} \bar{\psi}$ : for example, this is the case if $q=p^{2}$ and

$$
\phi: x \rightarrow\left(x, x^{(p)}\right), \quad \psi: x \rightarrow\left(x^{(p)}, x\right) .
$$

In general, $\operatorname{Im} \phi=\operatorname{Im} \psi$ means that $\psi$ is obtained from $\phi$ by applying a fixed field twist to each coordinate (and equating the $q$-power twist to 1 ).

Now let $\rho$ be a surjective homomorphism $S L_{2}(K)^{l} \rightarrow \bar{S}_{1} \ldots \bar{S}_{l}$. By the previous paragraph, we may assume that $S=\left(S L_{2}(q)\right) \phi \rho$ for some $\phi$ as above. Then $S$ lies in $\bar{S}_{\phi}=S L_{2}(K) \bar{\phi} \rho$, a closed subgroup of $\bar{S}_{1} \ldots \bar{S}_{l}$ isomorphic to $S L_{2}(K)$. Consequently the first aim of our strategy, to find a subgroup $S L_{2}(K)$ of $D$ containing $S$, is achieved in the situation of $4.2(\mathrm{i}, \mathrm{ii})$.

Observe that if $\psi$ is another embedding of $S L_{2}(q)$ such that $\operatorname{Im} \phi=\operatorname{Im} \psi$, then $S$ also lies in $\bar{S}_{\psi}$. Thus if $l>1$, then $S$ lies in many subgroups $S L_{2}(K)$ of $\bar{S}_{1} \ldots \bar{S}_{l}$, obtained from field twists of $\phi$. If we can show that one of these subgroups $\bar{S}_{\psi}$ has 
all its composition factors on $L(G)$ having high weight less than $q$, it will follow from 1.5 that $S$ and $\bar{S}_{\psi}$ fix the same subspaces of $L(G)$, contradicting our assumption $(\dagger)$, as desired for Theorem 1 . In the next few lemmas we show that this can usually be done.

Lemma 4.3. Suppose that case (5) of 3.5 holds (with $p \neq 2$ and $q>13$ ), and suppose also that 4.2(i) holds. Then either we can choose $\bar{S}$ with $S<\bar{S} \leq D$, such that every composition factor of $L(G) \downarrow \bar{S}$ has high weight less than q, or one of the following holds:

(i) $q=25, S<D \leq A_{1} A_{3} A_{3}$ with embedding $0^{2}, 3,3^{(5)}$ or $1,3,3^{(5)}$;

(ii) $q=25, S<D \leq A_{3} A_{4}$ with embedding $3^{(5)}, 4$ or $3^{(5)}, 3 / 0$.

Proof. We have $S<D \leq Y_{1} \ldots Y_{r}$, a product of classical groups $Y_{i}$ as in (5) of 3.5. In view of the natural embedding of $D$ in $Y_{1} \ldots Y_{r}$, we may replace $D$ by $Y_{1} \ldots Y_{r}$ in the discussion preceding the lemma. We know from that discussion that there is a closed subgroup $\bar{S} \cong S L_{2}(K)$ of $D$ containing $S$. Let $V_{i}$ be the usual module for $Y_{i}$. As usual, we describe the embedding of $\bar{S}$ in $Y_{1} \ldots Y_{r}$ by giving the composition factors of $V_{1} \downarrow \bar{S}, \ldots, V_{r} \downarrow \bar{S}$. We can certainly choose $\bar{S}$ so that all these composition factors have high weight less than $q$.

Suppose first that all the factors $Y_{i}$ are of type $A_{m}$ with $m \leq 4$. Projections of $\bar{S}$ in such factors are given by the following representations (up to field twists):

$$
\begin{array}{ll}
\bar{S} \rightarrow A_{1}: & 1 \text { or } 0^{2} \\
\bar{S} \rightarrow A_{2}: & 2,1 / 0 \text { or } 0^{3} \\
\bar{S} \rightarrow A_{3}: & 3,2 / 0,1 / 1^{\left(p^{i}\right)}, 1 \otimes 1^{\left(p^{i}\right)}, 1 / 0^{2} \text { or } 0^{4} \\
\bar{S} \rightarrow A_{4}: & 4,3 / 0,2 / 1^{\left(p^{i}\right)}, 2 / 0^{2}, 1 \otimes 1^{\left(p^{i}\right)} / 0,1 / 1^{\left(p^{i}\right)} / 0,1 / 0^{3} \text { or } 0^{5} .
\end{array}
$$

Each composition factor of $L(G) \downarrow Y_{1} \ldots Y_{r}$ occurs either in $L\left(Y_{i}\right)$ or in a tensor product of modules among the $V_{i}$ and $\bigwedge^{2} V_{i}$ and their duals (see [LS2, §2]). The highest weight of a composition factor of $\bigwedge^{2} V_{\bar{S}}(a)$ is $2 a-2$, and of $\bigwedge^{2}\left(1^{\left(p^{i}\right)} \otimes 1^{\left(p^{j}\right)}\right)$ (with $i>j$ ) is $2 p^{i}$.

Now $Y_{1} \ldots Y_{r}$ is one of

$$
A_{1} A_{3} A_{3}, A_{1} A_{2} A_{2} A_{2}, A_{1} A_{1} A_{2} A_{3}, A_{3} A_{4}, A_{1} A_{2} A_{4} .
$$

Suppose first that $V_{i} \downarrow \bar{S}$ has a composition factor $3^{\left(p^{j}\right)}$ or $4^{\left(p^{j}\right)}$ for some $i, j$. Then $p \geq 5$. Applying a field twist, as discussed in the preamble to the lemma, we can take the factor to be 3 or 4 . Arguing only from the above observations, we check that the only possibilities where a composition factor of high weight at least $q$ can occur in $L(G) \downarrow \bar{S}$ are:

(a) $\bar{S}<A_{1} A_{3} A_{3}$, with embedding $0^{2}, 3,3^{\left(p^{i}\right)}$ or $1^{\left(p^{j}\right)}, 3,3^{\left(p^{i}\right)}$ : here $L(G) \downarrow \bar{S}$ has possible highest weight $6 p^{i}$ or $p^{j}+4+4 p^{i}$;

(b) $\bar{S}<A_{3} A_{4}$, with embedding $3^{\left(p^{i}\right)}, 4$ or $3^{\left(p^{i}\right)}, 3 / 0$ : here $L(G) \downarrow \bar{S}$ has possible highest weight $6 p^{i}$ or $4 p^{i}+6$.

In both cases, the possible highest weight is at least $q$ only if $p=5$; and if $q=$ $5^{e}>25$, we can apply a field twist to take $i \leq e-2$ in all cases, giving highest weight less than $q$. Therefore a high weight at least $q$ can only occur if $q=25$ and conclusion (i) or (ii) of the lemma holds. 
We may now assume that no composition factor of $V_{i} \downarrow \bar{S}$ is 3 or 4 (or a twist thereof). From [LS2, 2.1 and 2.3] we see that the number of factors $V_{i}$ or $\bigwedge^{2} V_{i}$ in a tensor product occurring in $L(G) \downarrow Y_{1} \ldots Y_{r}$ is at most three; hence, referring to the list of possibilities for $Y_{1} \ldots Y_{r}$ above, we see that the highest weight occurring in $L(G) \downarrow \bar{S}$ is at most $6 p^{e-1}$, which is less than $q$ if $p>5$. Thus we may assume that $p=3$ or 5 .

For $p=5$, a high weight $q$ or more can be achieved only if three factors occur in a tensor product, each factor contributing $2 p^{e-1}$ (since otherwise the highest weight is at most $\left.4 p^{e-1}+2 p^{e-2}<q\right)$. Thus the embedding of $\bar{S}$ in $Y_{1} \ldots Y_{r}$ has three representations of the form $2^{\left(p^{e-1}\right)}$ or $1^{\left(p^{e-1}\right)} \otimes 1^{\left(p^{j}\right)}$. This forces $\bar{S}<A_{1} A_{2} A_{2} A_{2}$, with embedding $\left(0^{2}\right.$ or $\left.1^{\left(p^{i}\right)}\right), 2^{\left(p^{e-1}\right)}, 2^{\left(p^{e-1}\right)}, 2^{\left(p^{e-1}\right)}$. Applying a field twist, this becomes $\left(0^{2}\right.$ or $\left.1^{\left(p^{i+1}\right)}\right), 2,2,2$, and now $L(G) \downarrow \bar{S}$ has highest weight less than $q$.

Now consider $p=3$. Here $o(a) \geq q-1 \geq 26$, so we see from 3.2 and 3.4 that the semisimple group $D=C_{G}(a)^{\prime}$ cannot have rank 7 or 8 . Recall that the product $Y_{1} \ldots Y_{r}$ is as in 3.5(5), with factors $A_{m}(m \leq 4)$. Consequently $D$ lies in one of the following products:

$$
A_{1} A_{2} A_{3}, A_{1} A_{1} A_{2} A_{2}, A_{2} A_{2} A_{2}, A_{3} A_{3}, A_{2} A_{4}, A_{1} A_{1} A_{1} A_{3}, A_{1} A_{1} A_{4}
$$

Arguing as above, and applying a field twist if necessary, we see that the only case where $L(G) \downarrow \bar{S}$ can have highest weight at least $q$ is $\bar{S}<D \leq A_{2} A_{2} A_{2}$, with embedding $2,2^{(3)}, 2^{(9)}$ and $q=27$. But in this case $a \in C_{G}\left(A_{2} A_{2} A_{2}\right)=A_{2}=$ $C_{G}\left(E_{6}\right)$, which is not possible by 3.6. This completes the proof when all the $Y_{i}$ are of type $A_{m}$ with $m \leq 4$.

Next, suppose that $Y_{1} \ldots Y_{r}$ has a factor $A_{m}$ with $m \geq 5$, hence is $A_{2} A_{5}, A_{1} A_{1} A_{5}$ or $A_{1} A_{6}$. If the usual module for $A_{m}$ has all $\bar{S}$-composition factors of dimension at most 5 , then the arguments for the previous case (all factors $A_{n}, n \leq 4$ ) apply; hence the projection of $\bar{S}$ in $A_{m}$ is one of the following (up to twists):

$$
\begin{array}{ll}
\bar{S} \rightarrow A_{5}: & 5 \text { or } 2 \otimes 1^{\left(p^{i}\right)} \\
\bar{S} \rightarrow A_{6}: & 6,5 / 0 \text { or } 2 \otimes 1^{\left(p^{i}\right)} / 0 .
\end{array}
$$

By [LS2, 2.1 and 2.3], each composition factor of $L(G) \downarrow Y_{1} \ldots Y_{r}$ occurs in $L\left(Y_{i}\right)$, in $\bigwedge^{3} V_{i}$, or in a tensor product of modules among the $V_{i}$ and $\bigwedge^{2} V_{i}$ (and duals). Moreover, by [LS2, 2.13], the highest weights in $\bigwedge^{3} V_{\bar{S}}(r), \bigwedge^{2}\left(2 \otimes 1^{\left(p^{i}\right)}\right), \bigwedge^{2}\left(1 \otimes 2^{\left(p^{i}\right)}\right)$, $\bigwedge^{3}\left(2 \otimes 1^{\left(p^{i}\right)}\right), \bigwedge^{3}\left(1 \otimes 2^{\left(p^{i}\right)}\right)$ are $3 r-6,2 p^{i}+2,4 p^{i}, 3 p^{i}, 4 p^{i}+1$, respectively. Hence, as usual adjusting by a field twist if necessary, we see that $L(G) \downarrow \bar{S}$ has highest weight less than $q$ when $p>3$.

Now let $p=3$. Then $o(a) \geq q-1 \geq 26$, so by 3.2 and $3.4, D=C_{G}(a)^{\prime}$ has rank at most 6 . Consequently $\bar{S}$ must lie in a product $A_{1} A_{5}$, with embedding $0^{2}, 2 \otimes 1^{\left(3^{i}\right)}$ or $1^{\left(3^{j}\right)}, 2 \otimes 1^{\left(3^{i}\right)}$. Then $L(G) \downarrow \bar{S}$ has highest weight less than $q$, unless $i=e-1$; but in this case we apply a field twist to adjust the embedding to $0^{2}$ or $1^{\left(3^{k}\right)}, 2^{(3)} \otimes 1$, and now $L(G) \downarrow \bar{S}$ has highest weight less than $q$.

We have now handled all cases where the $Y_{i}$ are all of type $A_{m}$. It remains to consider $Y_{1} \ldots Y_{r}=A_{1} A_{1} D_{4}, A_{2} D_{4}$ or $A_{1} D_{5}$. The possible projections of $\bar{S}$ in the factor $D_{m}(m=4$ or 5$)$ are as follows (up to twists): 


$$
\begin{aligned}
\bar{S} \rightarrow D_{4}: & 6 / 0,4 / 2^{\left(p^{i}\right)}, 4 / 0^{3}, 3 / 3,2 / 2^{\left(p^{i}\right)} / 0^{2}, 2 / 1^{\left(p^{i}\right)} / 1^{\left(p^{i}\right)} / 0,2 / 0^{5} \\
& 1 / 1 / 1^{\left(p^{i}\right)} / 1^{\left(p^{i}\right)}, 1 / 1 / 0^{4}, 1 \otimes 1^{\left(p^{i}\right)} / 1^{\left(p^{j}\right)} \otimes 1^{\left(p^{k}\right)}, \\
& 1 \otimes 1^{\left(p^{i}\right)} / 1^{\left(p^{j}\right)} / 1^{\left(p^{j}\right)}, 3 \otimes 1^{\left(p^{i}\right)}, 1 \otimes 1^{\left(p^{i}\right)} / 0^{4}, 1 \otimes 1^{\left(p^{i}\right)} / 2^{\left(p^{j}\right)} / 0 \\
\bar{S} \rightarrow D_{5}: & 8 / 0,6 / 2^{\left(p^{i}\right)}, 6 / 0^{3}, 4 / 4^{\left(p^{i}\right)}, 4 / 2^{\left(p^{i}\right)} / 0^{2}, 4 / 0^{5}, 3 / 3 / 0^{2}, 2 / 2^{\left(p^{i}\right)} / 2^{\left(p^{j}\right)} / 0 \\
& 2 / 2^{\left(p^{i}\right)} / 1^{\left(p^{j}\right)} / 1^{\left(p^{j}\right)}, 2 / 2^{\left(p^{i}\right)} / 0^{4}, 2 / 1^{\left(p^{i}\right)} / 1^{\left(p^{i}\right)} / 0^{3}, 2 / 0^{7}, \\
& 1 / 1 / 1^{\left(p^{i}\right)} / 1^{\left(p^{i}\right)} / 0^{2}, 1 / 1 / 0^{6}, 2 \otimes 2^{\left(p^{i}\right)} / 0,3 \otimes 1^{\left(p^{i}\right)} / 0^{2}, \\
& 1 \otimes 1^{\left(p^{i}\right)} / 1^{\left(p^{j}\right)} \otimes 1^{\left(p^{k}\right)} / 0^{2}, 1 \otimes 1^{\left(p^{i}\right)} / 1^{\left(p^{j}\right)} / 1^{\left(p^{j}\right)} / 0^{2}, 1 \otimes 1^{\left(p^{i}\right)} / 0^{6} \\
& 1 \otimes 1^{\left(p^{i}\right)} / 4^{\left(p^{j}\right)} / 0,2 / 2^{\left(p^{i}\right)} / 1^{\left(p^{j}\right)} \otimes 1^{\left(p^{k}\right)}, 2 / 1^{\left(p^{i}\right)} \otimes 1^{\left(p^{j}\right)} / 0^{3} .
\end{aligned}
$$

By [LS2, 2.1 and 2.3],

$L(G) \downarrow A_{1} A_{1} D_{4}$ has nontrivial composition factors among $2 \otimes 0 \otimes 0,0 \otimes 2 \otimes 0$, $0 \otimes 0 \otimes \lambda_{2}, 0 \otimes 0 \otimes \lambda_{1}, 1 \otimes 1 \otimes 0,1 \otimes 1 \otimes \lambda_{i}, 1 \otimes 0 \otimes \lambda_{i}, 0 \otimes 1 \otimes \lambda_{i}, 0 \otimes 0 \otimes \lambda_{i}$ $(i=1,3,4)$,

$L(G) \downarrow A_{2} D_{4}$ has nontrivial composition factors $11 \otimes 0,0 \otimes \lambda_{2}, 0 \otimes \lambda_{j} \lambda_{i} \otimes \lambda_{j}$ $(i=1,2, j=1,3,4)$, and

$L(G) \downarrow A_{1} D_{5}$ has nontrivial composition factors $2 \otimes 0,0 \otimes \lambda_{2}, 0 \otimes \lambda_{i} 1 \otimes \lambda_{i}$ $(i=4,5)$.

As before, for each embedding of $\bar{S}$, the highest weight of $L(G) \downarrow \bar{S}$ can be worked out using [LS2, 2.13]. We find that in every case but one, applying a field twist if necessary, $L(G) \downarrow \bar{S}$ has highest weight less than $q$; the exceptional case is

$$
\left.q=25, S<A_{1} D_{5} \text {, embedding ( } 0^{2} \text { or } 1\right), 4 / 4^{(5)} .
$$

But in this case, $S$ also lies in $A_{1} A_{3} A_{3}$, as in conclusion (i) of the lemma.

Lemma 4.4. Suppose that case (5) of 3.5 holds, and also that 4.2(ii) holds. Then we can choose $\bar{S}$ with $S<\bar{S} \leq D$, such that every composition factor of $L(G) \downarrow \bar{S}$ has high weight less than $q$.

Proof. We have $S<\bar{S} \leq D$, and by applying a suitable field twist we may take it that $D$ has a factor $E$ with usual module $V$ such that $V \downarrow \bar{S}$ is as in Table 2 .

Suppose first that $p=5$. Then $E=A_{5}, A_{6}, D_{5}$ or $D_{6}$; as we are assuming 3.5(5) holds, $D$ lies in $A_{2} A_{5}, A_{1} A_{1} A_{5}, A_{1} A_{6}$ or $A_{1} D_{5}$. We may take the embedding of $\bar{S}$ in these groups to be one of the following:

$$
\begin{gathered}
\bar{S}<A_{2} A_{5} \text { : projection }\left(0^{3}, 1^{\left(5^{i}\right)} / 0 \text { or } 2^{\left(5^{i}\right)}\right), 3 / 1^{(5)} \quad\left(\text { with } 5^{i}<q\right) \\
\bar{S}<A_{1} A_{1} A_{5} \text { : embedding }\left(0^{2} \text { or } 1^{\left(5^{i}\right)}\right),\left(0^{2} \text { or } 1^{\left(5^{j}\right)}\right), 3 / 1^{(5)} \quad\left(\text { with } 5^{i}, 5^{j}<q\right) \\
\bar{S}<A_{1} A_{6} \text { : embedding }\left(0^{2} \text { or } 1^{\left(5^{i}\right)}\right),\left(3 / 1^{(5)} / 0 \text { or } 2 / 1 \otimes 1^{(5)}\right)\left(\text { with } 5^{i}<q\right) \\
\bar{S}<A_{1} D_{5} \text { : embedding }\left(0^{2} \text { or } 1^{\left(5^{i}\right)}\right),\left(3 \otimes 1^{(5)} / 0^{2} \text { or } 2^{2} / 1 \otimes 1^{(5)}\right)\left(\text { with } 5^{i}<q\right)
\end{gathered}
$$

In all cases we find that the highest weight of $\bar{S}$ on $L(G)$ is less than $q$, as required.

Now suppose $p=3$. Then $E=A_{r}(3 \leq r \leq 6), D_{4}$ or $D_{5}$. As in the proof of 4.3 , and using 3.4 to rule out all rank 7 possibilities, we see that $D$ lies in one of the following subgroups $Y_{1} \ldots Y_{r}$ :

$$
A_{1} A_{2} A_{3}, A_{3} A_{3}, A_{2} A_{4}, A_{1} A_{1} A_{1} A_{3}, A_{1} A_{1} A_{4}, A_{1} A_{5}, A_{6}, A_{1} A_{1} D_{4}, A_{2} D_{4}, A_{1} D_{5} .
$$

If $D \leq A_{1} A_{2} A_{3}$, the conclusion is clear unless the embedding of $\bar{S}$ is $\left(0^{2}\right.$ or $\left.1^{\left(3^{i}\right)}\right)$, $2^{\left(3^{e-1}\right)}, 1 / 1^{(3)}$ (recall that $\left.q=3^{e}\right)$. Applying a field twist, we can change $\bar{S}$ to take the embedding to be $\left(0^{2}\right.$ or $\left.1^{\left(3^{i+1}\right)}\right), 2,1^{(3)} / 1^{(9)}$; this gives the conclusion unless 
$i+1=e$, in which case we further twist to replace the $1^{\left(3^{i+1}\right)}$ by just 1 . Now the weights on $L(G)$ are less than $q$, as required.

This argument applies to all cases where the factors $Y_{i}$ are all of type $A_{k}$, except for $A_{3} A_{3}$, in which case $\bar{S}$ could be indecomposable on both natural modules for the factors. Here the embedding is $1 / 1^{(3)},\left(1 / 1^{(3)}\right)^{\left(3^{i}\right)}$, and we can assume that $3^{i}<q$. The weights are all less than $q$ unless $3^{i+1}=q$. We can now change $\bar{S}$ by applying a 3 -power twist to take the embedding to be $1^{(3)} / 1^{(9)}, 1 / 1^{(3)}$, for which the weights are less than $q$.

It remains to handle the cases where some $Y_{i}$ is $D_{4}$ or $D_{5}$. For these the weights of $\bar{S}$ on $L(G)$ are less than $q$ unless possibly $\bar{S}<A_{1} D_{5}$ with embedding $1 \otimes 1^{(3)} / 1^{\left(3^{i}\right)} / 1^{\left(3^{i}\right)} / 0^{2}$ or $1 \otimes 1^{(3)} / 1^{\left(3^{i}\right)} \otimes 1^{\left(3^{l}\right)} / 0^{2}(i<l)$ in the $D_{5}$ factor. In the first case, $\bar{S}<A_{1} A_{1} D_{3}$, a case handled above. In the second case, if the factor $1^{\left(3^{i}\right)} \otimes 1^{\left(3^{l}\right)}$ splits off then $\bar{S}<A_{1} A_{1} A_{1} D_{3}$, a case already considered. Otherwise, by 1.2 , we have $l=i+1$. The weights of $\bar{S}$ on $L(G)$ are less than $q$ unless $i+1=e$, in which case we twist to embedding $1^{(3)} \otimes 1^{(9)} / 1 \otimes 1^{(3)} / 0^{2}$, for which the weights are all less than $q$.

Continue to assume that $p \neq 2, q>13$. By 3.5-3.7 and the previous two lemmas, we may now assume that either we are in one of the exceptional cases of $4.2(\mathrm{iii}, \mathrm{iv}, \mathrm{v})$, 4.3(i,ii), or $D$ and $q$ are as in Table 3 .

TABLE 3

\begin{tabular}{ll}
$D$ & $q$ \\
\hline$A_{1} A_{2} A_{5}, A_{1} E_{6}, A_{1} D_{6}$ & 17,19 \\
$A_{1} A_{1} D_{5}, A_{7}, A_{2} D_{5}, A_{3} D_{4}$ & $17,19,23,25$ \\
$D_{6}$ & $q$ prime or $q=25$ \\
\hline
\end{tabular}

Lemma 4.5. Suppose that $q$ is prime and $D$ is as in Table 3, with $D \neq A_{1} E_{6}$. Then we can choose $\bar{S} \cong A_{1}(K)$ such that $S<\bar{S} \leq D$ and the highest weight of $L(G) \downarrow \bar{S}$ is less than $q$, except in the following case:

$$
q=17, S<D=A_{1} D_{6} \text { or } D_{6} \text {, with embedding } 10 / 0 \text { in the } D_{6} \text { factor. }
$$

Proof. Since $q=p \geq 17$, it follows from 1.2 that $S$ acts completely reducibly on the usual module for each factor of $D$. Hence there is a subgroup $\bar{S} \cong S L_{2}(K)$ of $D$ containing $S$ (see the preamble to 4.3). We see as in the proof of the previous lemma that either we can take the highest weight of $\bar{S}$ to be less than $q$, or $D$ has a factor $D_{6}$ with $S$ projecting via 10/0, as in the conclusion.

Lemma 4.6. If $q=25$ and $D$ is as in Table 3, then either we can choose $\bar{S} \cong$ $A_{1}(K)$ such that $S<\bar{S} \leq D$ and the highest weight of $L(G) \downarrow \bar{S}$ is less than $q$, or one of the following holds:

(i) $D$ has a factor $D_{5}$ or $D_{6}$, and the embedding of $S$ in this factor is $4 / 4^{(5)} / 0^{m}$ $(m=0$ or 2$)$;

(ii) $S<D=A_{3} D_{4}$, with embedding $3^{(5)}, 1^{(5)} \otimes 3$ or $3^{(5)}, 3 / 3$ or $3^{(5)}, 4 / 2$ or $3^{(5)}, 4 / 2^{(5)}$ or $3^{(5)}, 4 / 0^{3}$;

(iii) $S<D=A_{7}$, with embedding $3 / 3^{(5)}$.

Proof. If $S$ is not completely reducible on the usual module for one of the factors of $D$, then one of the possibilities given in Table 2 of 4.2 holds, from which we easily 
see that $\bar{S}$ exists, with all weights on $L(G)$ less than $q$. Otherwise, $S$ is completely reducible in all factors of $D$. The only way the desired $\bar{S}$ can fail to exist is for some $V_{i} \downarrow S$ to have a composition factor $3^{(5)}$ or $4^{(5)}$ which cannot be excluded by applying a field twist; all such possibilities are listed under conclusions (i)-(iii).

The next lemma deals with the possibility $D=A_{1} E_{6}$ excluded in 4.5 .

Lemma 4.7. Theorem 1 holds if $D=A_{1} E_{6}$.

Proof. Here $q=17$ or 19 , and by $3.4, o(a) \leq 6$. Consequently the group $A$ must be $U_{3}(17)$ or $L_{3}(19)$. Observe that the projection of $S$ to the factor $E_{6}$ is a group $S_{0} \cong L_{2}(p)$. Pick $u \in S$ of order $p$, and write $u=u_{1} u_{0}$ with $u_{1} \in A_{1}, u_{0} \in S_{0}<E_{6}$.

Assume first that $u_{0}$ is a regular or semiregular element of $E_{6}$. Now the projection of $C_{A}(a)$ to $E_{6}$ in fact contains $P G L_{2}(p)$. Under these circumstances, [ST2, Theorem 1] implies that there is a subgroup $\bar{S} \cong A_{1}(K)$ of $E_{6}$ containing $S_{0}$. Moreover, by [LT, Table 3], $\bar{S}$ is determined up to $E_{6}$-conjugacy; the composition factors of $L\left(E_{6}\right) \downarrow \bar{S}$ are as in [Se2, p.65]. It follows that $L\left(E_{6}\right) \downarrow \bar{S}$ has a unique composition factor with high weight 16 . Hence the same is true of $L\left(E_{8}\right) \downarrow S$. Consequently $L\left(E_{8}\right) \downarrow A$ has a composition factor $V_{A}(a a)$ with $2 a \geq 16$. By 1.5 , $V_{A}(a a)$ has dimension either $(a+1)^{3}$ or $(a+1)^{3}-(p-a-1)^{3}$. Since this is at most 248, we must have $p=17$ and $a=8$, in which case $V_{A}(a a)$ has dimension 217. Now $V_{A}(88) \downarrow S$ has two composition factors of high weight 15 ; moreover, $S<A_{1} \bar{S}<A_{1} E_{6}$, and

$$
L(G) \downarrow A_{1} E_{6}=L\left(A_{1}\right) / L\left(E_{6}\right) / 1 \otimes \lambda_{1} / 1 \otimes \lambda_{6} / 0 \otimes \lambda_{1} / 0 \otimes \lambda_{6} /(1 \otimes 0)^{2} / 0
$$

(see [LS2, §2]). It follows that the restriction of the $A_{1} E_{6}$-module $1 \otimes \lambda_{1}$ to $S$ must have a composition factor of high weight 15. By [LS2, 2.5], we have $\lambda_{1} \downarrow S_{0}=$ $16 / 8 / 0$ or $12 / 8 / 4$; hence, by the previous sentence, in fact $\lambda_{1} \downarrow S_{0}=16 / 8 / 0$. Then $1 \otimes \lambda_{1} \downarrow S$ has two composition factors 15 , whence $L(G) \downarrow S$ has four composition factors 15 . This implies that $L(G) \downarrow A$ has a further factor $V_{A}(b c)$ with $b+c \geq 15$; but then $\operatorname{dim} V_{A}(88)+\operatorname{dim} V_{A}(b c)>248$, a contradiction.

Now suppose that $u_{0}$ is not regular or semiregular in $E_{6}$. Then $u_{0}$ lies in the centralizer in $E_{6}$ of a semisimple element, hence lies in a subsystem subgroup $Y=$ $D_{5}, A_{1} A_{5}$ or $A_{2} A_{2} A_{2}$. Since $p \geq 17$, it follows using 1.7 that $u_{0}$ lies in a subgroup $\bar{S} \cong A_{1}(K)$ of $Y$ such that $\bar{S}$ has restricted composition factors on the usual modules for all factors of $Y$. If we write $M$ for the factor $A_{1}$ of $D=A_{1} E_{6}$, then $u$ lies in a diagonal subgroup $\tilde{S} \cong A_{1}(K)$ of $M \bar{S}$ (where neither projection involves a twist). The restrictions $L\left(E_{8}\right) \downarrow M Y$ are given by [LS2, 2 ], and we deduce from this that all composition factors of $L\left(E_{8}\right) \downarrow \tilde{S}$ are restricted. It now follows from [LST, 1.14] that if $U$ is a connected unipotent subgroup of $\tilde{S}$ containing $u$, then $u$ and $U$ fix the same subspaces of $L\left(E_{8}\right)$, contrary to $(\dagger)$.

To complete the proof of Theorem 1 for $q>13, p \neq 2$, all that remains is to handle the exceptional cases in the conclusions of 4.2, 4.3, 4.5 and 4.6.

Lemma 4.8. None of the exceptional cases in 4.2(iii, iv, v) can occur.

Proof. Consider first case 4.2(iii); here $q=27$ and $S<A_{5}<D$ with embedding $1 / 1^{(3)} / 1^{(9)}$. From [LS2, Table 8.1] we have

$$
L(G) \downarrow A_{5}=L\left(A_{5}\right) / \lambda_{1}^{6} / \lambda_{2}^{3} / \lambda_{3}^{2} / \lambda_{4}^{3} / \lambda_{5}^{6} / 0^{11} .
$$


Hence

$$
\begin{aligned}
L(G) \downarrow S= & \left(1 \otimes 1^{(3)} \otimes 1^{(9)}\right)^{2} /\left(1 \otimes 1^{(3)}\right)^{8} /\left(1 \otimes 1^{(9)}\right)^{8} /\left(1^{(3)} \otimes 1^{(9)}\right)^{8} / \\
& 1^{16} /\left(1^{(3)}\right)^{16} /\left(1^{(9)}\right)^{16} / 2 / 2^{(3)} / 2^{(9)} / 0^{31} .
\end{aligned}
$$

Recall that $S<A=A_{2}^{\epsilon}(27)$ or $B_{2}(27)$. If $A=B_{2}(27)$ then $S$ contains a conjugate $b$ of $a$ (of order 28). However from the above restriction of $L(G)$ to $S$, we see that $\operatorname{dim} C_{L(G)}(b)=34$, whereas $C_{G}(a)$ contains $A_{5}$, of dimension 35 , a contradiction.

Therefore $A=A_{2}^{\epsilon}(27)$. Since $L(G) \downarrow S$ has two factors $1 \otimes 1^{(3)} \otimes 1^{(9)}$, it follows that $L(G) \downarrow A$ has a factor $\alpha \otimes \beta^{(3)} \otimes \gamma^{(9)}$ and its dual, where $\alpha, \beta, \gamma \in\{10,01\}$. Moreover,

$$
\left(\alpha \otimes \beta^{(3)} \otimes \gamma^{(9)}\right) \downarrow S=1 \otimes 1^{(3)} \otimes 1^{(9)} / 1 \otimes 1^{(3)} / 1 \otimes 1^{(9)} / 1^{(3)} \otimes 1^{(9)} / 1 / 1^{(3)} / 1^{(9)} / 0 .
$$

Now $V_{A}(22) \downarrow S$ has three composition factors of high weight 2 , hence is not present in $L(G) \downarrow A$. In order to accomodate the further six composition factors $1 \otimes 1^{(3)}$ in $L(G) \downarrow S$, it follows that $L(G) \downarrow A$ must have three factors of the form $\delta \otimes \epsilon^{(3)}$ with $\delta, \epsilon \in\{10,01\}$, together with their duals; similarly for the other 2-fold twists.

The composition factors found so far for $L(G) \downarrow A$ account for all composition factors of $L(G) \downarrow S$ except $1^{2} /\left(1^{(3)}\right)^{2} /\left(1^{(9)}\right)^{2} / 2 / 2^{(3)} / 2^{(9)} / 0^{11}$; in order to accomodate these, $L(G) \downarrow A$ must have further factors $11 / 11^{(3)} / 11^{(9)} / 00^{8}$.

Now $a$ is an element of order 26 or 28 in $A$ of the form $\operatorname{diag}\left(\omega, \omega, \omega^{-2}\right)$ for some $\omega \in K^{*}$. We calculate that $\operatorname{dim} C_{V_{A}(\lambda)}(a)$ is at most 1 if $\lambda=\alpha \otimes \beta^{(3)} \otimes \gamma^{(9)}$, is 0 if $\lambda=\delta \otimes \epsilon^{(3)}$, and is 4 if $\lambda=11$. Hence from the above we see that $\operatorname{dim} C_{L(G)}(a) \leq 22$. This is a contradiction, as $C_{G}(a)$ contains $A_{5}$. This completes the argument for the case 4.2 (iii).

The case $4.2(\mathrm{iv})$ is handled similarly. Here $S<D=A_{1} D_{5}$, and from [LS2, §2],

$$
\begin{aligned}
L(G) \downarrow A_{1} D_{5}= & L\left(A_{1}\right) / L\left(D_{5}\right) /\left(1 \otimes \lambda_{1}\right)^{2} / 1 \otimes \lambda_{4} / 1 \otimes \lambda_{5} /\left(0 \otimes \lambda_{4}\right)^{2} / \\
& \left(0 \otimes \lambda_{5}\right)^{2} /\left(0 \otimes \lambda_{1}\right)^{2} /(1 \otimes 0)^{4} / 0^{4} .
\end{aligned}
$$

From the proof of 4.2 , we see that the projection of $S$ in the $D_{5}$ factor is as in case (c) for $p=3$, namely $\left(1 \otimes 1^{(3)} / 0 / 1 \otimes 1^{(3)}\right) \perp V_{1}$, the space $1 \otimes 1^{(3)}$ being a singular 4 -space. We now calculate that

$$
\begin{aligned}
L(G) \downarrow S= & \left(1 \otimes 1^{(3)} \otimes 1^{(9)}\right)^{8} /\left(2 \otimes 1^{(9)}\right)^{2} /\left(2^{(3)} \otimes 1^{(9)}\right)^{2} / 2 \otimes 2^{(3)} / \\
& \left(1 \otimes 1^{(3)}\right)^{16} / 2^{7} /\left(2^{(3)}\right)^{7} / 2^{(9)} /\left(1^{(9)}\right)^{12} / 0^{18} .
\end{aligned}
$$

If $A=B_{2}(27)$ then, as above, $S$ contains a conjugate $b$ of $a$, and from this restriction we see that $C_{L(G)}(b)$ has dimension 34 , which is a contradiction as $C_{G}(a)^{\prime}=A_{1} D_{5}$. Thus $A=A_{2}^{\epsilon}(27)$. As in the previous case, $L(G) \downarrow A$ must have a composition factor $\alpha \otimes \beta^{(3)} \otimes \gamma^{(9)}$ with $\alpha, \beta, \gamma \in\{10,01\}$. But the restriction of this to $S$ has a composition factor $1 \otimes 1^{(9)}$, which is not present in $L(G) \downarrow S$.

Finally, consider $4.2(\mathrm{v})$. Here we calculate that

$$
\begin{aligned}
L(G) \downarrow S= & \left(1 \otimes 2^{(3)} \otimes 1^{(9)}\right)^{2} /\left(1 \otimes 1^{(3)} \otimes 1^{(9)}\right)^{2} /\left(1 \otimes 2^{(3)}\right)^{4} / \\
& \left(2^{(3)} \otimes 1^{(9)}\right)^{4} /\left(1 \otimes 1^{(3)}\right)^{6} /\left(1 \otimes 1^{(9)}\right)^{4} /\left(1^{(3)} \otimes 1^{(9)}\right)^{7} / \\
& 2 /\left(2^{(3)}\right)^{8} /\left(2^{(9)}\right)^{3} / 1^{4} /\left(1^{(3)}\right)^{12} /\left(1^{(9)}\right)^{8} / 0^{8} .
\end{aligned}
$$

We obtain the usual contradiction if $A=B_{2}(27)$, so assume $A=A_{2}^{\epsilon}(27)$. In order to produce $\left(1 \otimes 2^{(3)} \otimes 1^{(9)}\right)^{2}$ for $S, L(G) \downarrow A$ must have a factor $\lambda=\alpha \otimes \beta^{(3)} \otimes \gamma^{(9)}$ and its dual, where $\alpha, \gamma \in\{10,01\}, \beta \in\{20,02\}$ (note that $L(G) \downarrow A$ cannot have factors $\alpha \otimes 22^{(3)}$ or $\alpha \otimes 11^{(3)} \otimes \gamma^{(9)}$, as these are incompatible with $\left.L(G) \downarrow S\right)$. Removing the composition factors of $\left(\lambda+\lambda^{*}\right) \downarrow S$ from $L(G) \downarrow S$ still leaves two 
factors $2^{(3)} \otimes 1^{(9)}$. To accommodate these, $L(G) \downarrow A$ must have $\delta^{(3)} \otimes \mu^{(9)}$ and its dual, where $\delta \in\{20,02,11\}, \mu \in\{10,01\}$. Removing the restrictions of these to $S$ leaves a further one or three factors $1^{(3)} \otimes 1^{(9)}$. At least one of these must occur in the restriction of a self-dual factor of $L(G) \downarrow A$, which must be $22^{(3)}$. Removing all $S$-composition factors of this from what is left of $L(G) \downarrow S$ leaves $\left(1 \otimes 2^{(3)}\right)^{2} /\left(2^{(3)}\right)^{1} / \ldots$. The factors $1 \otimes 2^{(3)}$ must occur in the restriction of $\phi \otimes \psi^{(3)}$ and its dual, where $\phi \in\{10,01\}, \psi \in\{20,02,11\}$; but this restriction has two composition factors $2^{(3)}$, which is a contradiction. This completes the proof.

Lemma 4.9. None of the exceptional cases in 4.3 or 4.6 can occur.

Proof. Suppose false, so $q=25$. By considering the restriction of $L(G)$ to the subsystem subgroup containing $D$ given in 4.3 or 4.6 , we see that $L(G) \downarrow S$ has a composition factor $4 \otimes k^{(5)}$ or $4^{(5)} \otimes k$, where $k \in\{3,4\}$. Applying a twist, we may assume the factor is $4 \otimes k^{(5)}$. Recall that $X$ has a subgroup $A \cong A_{2}^{\epsilon}(q)$ or $B_{2}(q)$ which contains $S$. Let $W$ be a composition factor of $L(G) \downarrow A$ such that $W \downarrow S$ has $4 \otimes k^{(5)}$ as a composition factor.

Observe first that $W$ is not restricted, since the highest weight of $V_{A}(a b) \downarrow S$ $(a, b \leq 4)$ is $a+b$. Hence $W=a b \otimes c d^{(5)}$, with $a, b, c, d \leq 4$.

If $a+b<4$ then $c d^{(5)} \downarrow S$ must have a composition factor $l \otimes k^{(5)}=l^{(25)} \otimes k^{(5)}$, with $1 \leq l \leq 4$. Then $5(c+d) \geq 25 l+5 k \geq 40$, forcing $c=d=4$. However, 44 (the Steinberg module) has dimension $5^{3}$ (for $A=A_{2}^{\epsilon}(25)$ ) or $5^{4}$ (for $A=B_{2}(25)$ ), so $a b \otimes 44^{(5)}$ has dimension greater than 248 , a contradiction.

Therefore $a+b \geq 4$. Similarly we see that $c+d \geq k$. For $A=A_{2}^{\epsilon}(q)$ it is now easy to check that $\operatorname{dim} W>248$ if $W$ is self-dual (i.e. if $a=b$ and $c=d$ ), and that $\operatorname{dim}\left(W+W^{*}\right)>248$ otherwise. And for $A=B_{2}(q)$ we check that $\operatorname{dim} W>248$, by counting conjugates of subdominant weights and using $[\mathrm{Pr}]$.

Lemma 4.10. The exceptional case in 4.5 cannot occur.

Proof. Suppose false, so that $q=17$ and $S<D=D_{6}$ or $A_{1} D_{6}$ with embedding $\left(0^{2}\right), 10 / 0$ or $1,10 / 0$. By [LS2, $\left.\S 2\right]$, we have

$$
L(G) \downarrow A_{1} D_{6}=L\left(A_{1} D_{6}\right) /\left(1 \otimes \lambda_{1}\right)^{2} / 1 \otimes \lambda_{5} / \lambda_{6}^{2} / 0^{3} .
$$

Moreover, by [LS2, 2.13], $\lambda_{5} \downarrow S \cong \lambda_{6} \downarrow S=15 / 9 / 5$. Therefore $L(G) \downarrow S$ has a composition factor with high weight 15 or 16 . If $A=B_{2}(17)$ then we can take $a$ to have order 18, and the conclusion follows from the proof of 3.7. Therefore $A=A_{2}^{\epsilon}(17)$. Now $L(G) \downarrow A$ must have a composition factor $V_{A}(a b)$ with $a+b=15$ or 16. By 1.8, this forces $a=b=8$, in which case $\operatorname{dim} V_{A}(a b)=217$. Now $V_{A}(88)=W_{A}(88) / W_{A}(77)$, from which we calculate that

$$
V_{A}(88) \downarrow S=16^{1} / 15^{2} / 14^{2} / \ldots
$$

To obtain the factor 16 , the embedding of $S$ in $A_{1} D_{6}$ must be $1,10 / 0$, whence $L(G) \downarrow S=16^{1} / 15^{2} / 14^{3} / \ldots$ The factor 14 not occurring in $V_{A}(88) \downarrow S$ must occur in the restriction of a self-dual $A$-composition factor of $L(G)$, which must be $V_{A}(77)$. However, $\operatorname{dim} V_{A}(88)+\operatorname{dim} V_{A}(77)>248$, which is a contradiction.

This completes the proof of Theorem 1 for $p \neq 2, q>13$. 


\section{Completion of the Proof of Theorem 1 For $p \neq 2$}

In this section we complete the proof of Theorem 1 for $p \neq 2$. Since $q>9$ by hypothesis, by the previous section it remains only to handle the cases $q=11$ and $q=13$. Thus let $G=E_{8}$ and suppose that $X=X(q)<G$ with $q=11$ or 13 . By the hypothesis of Theorem $1, X \neq L_{2}(q)$, so $X$ contains a subgroup $A$ isomorphic to one of the following groups:

$$
A_{2}^{\epsilon}(11), A_{2}^{\epsilon}(13), B_{2}(11), B_{2}(13) .
$$

As in the previous section, by 3.2 we may assume

$(\dagger)$ there are no subgroups $Y \leq X, Z=Z^{0} \leq G$, such that every $Y$-invariant subspace of $L(G)$ is $Z$-invariant and $X \cap Z \not \leq Z(X)$.

$A$ in a series of lemmas. The most complicated cases occur when $A=L_{3}(13)$ or $U_{3}(11)$, and we leave these until the final lemma.

We begin with a useful fact about self-dual indecomposables for $S L_{2}(p)$.

Lemma 5.1. Let $S=S L_{2}(p)(p=11$ or 13$)$, and let $V$ be a self-dual indecomposable $K S$-module of the form $4 / 4(p=11)$ or $5 / 5(p=11$ or 13$)$. Then $S<S O(V)$ only if $V=5 / 5$ with $p=11$; otherwise $S<S p(V)$.

Proof. Suppose $S<S O(V)=S O_{2 r}(r=4$ or 5$)$. Then $S<P_{r}=Q L$, the stabilizer of a maximal totally singular subspace, with unipotent radical $Q$; moreover, $Q$ is abelian, and $Q \downarrow S \cong \bigwedge^{2} V(r)$, which is $6 / 2$ if $r=4$ and $8 / 4 / 0$ if $r=5$. Since $V$ is indecomposable, $S$ does not lie in a conjugate of $L$, so $H^{1}(Q, S) \neq 0$. It follows from $1.2(\mathrm{i})$ that $r=5$ and $p=11$, as required.

Lemma 5.2. Theorem 1 holds if $A=S L_{3}(13)$ or $S U_{3}(11)$.

Proof. Write $Z(A)=\langle t\rangle$, a group of order 3 . We claim that $C_{G}(t)=A_{2} E_{6}$ or $A_{8}$. Now $A<C_{G}(t)=T_{k} E$, where $E$ is a semisimple subsystem subgroup of $G$. Then $A<E=C_{G}(t)^{\prime}$, whence $t \in Z(E)$. Therefore $E$ has a simple factor of type $A_{2}, A_{5}, E_{6}$ or $A_{8}$. In the last case the claim holds. If there is an $E_{6}$ factor or a unique $A_{2}$ factor, then $C_{G}(t)$ contains the centralizer of this factor, whence $C_{G}(t)=A_{2} E_{6}$, as in the claim. If there is an $A_{5}$ factor, this has centralizer $A_{2} A_{1}$ in $G$, whence $C_{G}(t)$ has an $A_{2}$ factor. It remains to show that $C_{G}(t)$ cannot have more than one $A_{2}$ factor. If there is more than one, then the normalizer of the product of all the $A_{2}$ factors is $A_{2}^{4}$, whence $C_{G}(t)=A_{2}^{4}$, contradicting 1.14. Thus $C_{G}(t)=A_{2} E_{6}$ or $A_{8}$, as claimed.

Suppose first that $C_{G}(t)=A_{8}$; in fact, then $C_{G}(t)=S L_{9} / Z_{3}$. But this group cannot contain $A$, since if it did, the preimage of $Z(A)$ in $S L_{9}$ would be cyclic of order 9 , which is clearly impossible.

Now assume $C_{G}(t)=A_{2} E_{6}$. If $A$ lies in the $A_{2}$ factor, then these groups fix the same subspaces of $L(G)$; hence $A$ projects nontrivially to the $E_{6}$ factor. Choose $a \in A$ of order 4 such that $C_{A}(a)^{(\infty)}=S \cong S L_{2}(p)$. The projection of $S$ in $E_{6}$ centralizes an involution, hence lies in a subgroup $A_{1} A_{5}$ or $T_{1} D_{5}$; hence $S$ lies in $T_{1} A_{1} A_{1} A_{5}$ or $T_{1} A_{1} T_{1} D_{5}$. The restriction of $L(G)$ to these subgroups is given by [LS2, §2], and for all but one possible embedding of $S$ we conclude that $S<\bar{S} \cong S L_{2}(K)$, where all high weights of $L(G) \downarrow \bar{S}$ are less than $q$, giving the usual contradiction to $(\dagger)$. The one exception is $S<A_{1} D_{5}$ with embedding $1,8 / 0$ or $0^{2}, 8 / 0$ (note that projection into $D_{5}$ is not the indecomposable $4 / 4$, by 5.1 ). In this case, let $S_{0}$ be the projection of $S$ in $E_{6}$, and consider the action of $S_{0}$ on 
the 27-dimensional module $V_{27}=V_{E_{6}}\left(\lambda_{1}\right)$. Since $V_{27} \downarrow D_{5}=\lambda_{1} / \lambda_{4} / 0$, there is a composition factor of $V_{27} \downarrow S_{0}$ of high weight 10 (see [LS2, 2.13]). Therefore if $A_{0}$ denotes the projection of $A$ in $E_{6}, V_{27} \downarrow A_{0}$ must have a composition factor $V_{A_{0}}(a b)$ with $a+b \geq 10$, which is impossible by 1.9 .

Lemma 5.3. Theorem 1 holds if $A=L_{3}(11)$ or $U_{3}(13)$.

Proof. The arguments are very similar to those of the previous lemma, and we give just a sketch. Pick $a \in A$ of order 10 or 14 (order 10 if $A=L_{3}(11)$, order 14 if $\left.A=U_{3}(13)\right)$, such that $C_{A}(a)^{(\infty)}=S \cong S L_{2}(p)$. As usual, by $(\dagger)$ we may suppose that there is no subgroup $\bar{S} \cong S L_{2}(K)$ containing $S$ and having all composition factors on $L(G)$ of high weight less than $q$. If $C_{G}(a)=T_{1} D$ with $D$ semisimple, then 3.4 implies that $D=A_{3} A_{4}, A_{1} A_{2} A_{4}, A_{2} A_{1} A_{1} A_{3}$ or $A_{1} A_{1} A_{5}$; in each case $\bar{S}$ as above exists. Otherwise, writing $o(a)=2 r$, and working in $C_{G}\left(a^{r}\right)=A_{1} E_{7}$ or $D_{8}$, we find that $S<C_{G}(a)^{\prime}=A_{6}, D_{6}, D_{5}$ or $A_{1} D_{5}$ (in all other cases, $\bar{S}$ as above exists).

If $S<A_{6}$, the non-existence of $\bar{S}$ as above (having all weights less than $q$ ) implies that the embedding is given by the representation 6 of $S$, and $p=11$. But the image of this representation of $S L_{2}(11)$ in $A_{6}$ is $L_{2}(11)$, so this is impossible.

If $S<D_{6}$, the embedding must be $10 / 0,8 / 2$ or $5 / 5$ (an indecomposable extension in the last case, with $p=11$ by 5.1 ). (Note that the embedding $8 / 0^{3}$ has image group $L_{2}(p)$ (see [LS2, 2.13]), and $4 / 4 / 0^{2}$ is out by 5.1.) By [LS2, Table 8.1],

$$
L(G) \downarrow D_{6}=\lambda_{2} / \lambda_{1}^{4} / \lambda_{5}^{2} / \lambda_{6}^{2} / 0^{6},
$$

and the restriction to $S$ can be worked out from this using [LS2, 2.13]. If the embedding of $S$ is 10/0 then $L(G) \downarrow S$ has at least five composition factors of high weight 10 , which is not possible by 1.9 . If the embedding is $8 / 2$ with $p=13$, then $L(G) \downarrow S$ has four composition factors of high weight 11, and none of high weight 12 ; and if the embedding is $8 / 2$ with $p=11$, then $L(G) \downarrow S=10^{2} / 9^{8} / \ldots$. Both of these are impossible, by 1.6. Now suppose the embedding is $5 / 5$ with $p=11$. We find that $L(G) \downarrow S=10^{1} / 9^{2} / 8^{7} / \ldots$. It follows that $L(G) \downarrow A$ must have a self-dual composition factor giving a 10 on restriction to $S$. This must be $V_{A}(55)$; by 1.9 , it has dimension 91 , and restricts to $S$ as $10^{1} / 9^{2} / 8^{2} / \ldots$. The remaining composition factors of $L(G) \downarrow A$ restrict to $S$ to give a further $8^{5}$, so it follows that one of them is $V_{A}(44)$, which has dimension 125 by 1.8 . There remain composition factors of $L(G) \downarrow A$, with restriction to $S$ giving $8^{4}$, and of total dimension $248-91-125=32$. This is clearly impossible, by 1.8 .

Finally, if $S<A_{1} D_{5}$, the embedding must be $1,8 / 0$ (the $4 / 4$ indecomposable with $p=11$ is not possible by 5.1). If $p=13$ we find that $L(G) \downarrow S$ has $11^{2} / 10^{6}$ and no 12 ; and if $p=11$, it has $10^{5}$. Both of these are incompatible with 1.9 .

Lemma 5.4. Theorem 1 holds if $A=B_{2}(11)$ or $B_{2}(13)$.

Proof. Pick an element $a \in A$ of order 10 or 12 such that $C_{A}(a)^{(\infty)}=S \cong S L_{2}(p)$. As in the previous proof, we have $S<C_{G}(a)^{\prime}=A_{6}, D_{6}$ or $A_{1} D_{5}$. Since $A$ contains a product of two commuting conjugates of $S$, there is a conjugate $b$ of $a$ lying in $S$.

Suppose $S<D_{6}$; as before, the embedding must be $10 / 0,8 / 2$ or $5 / 5(p=11)$. In the first two cases, $C_{V_{D_{6}}\left(\lambda_{i}\right)}(b)$ has dimension at most $4,14,0,0$ according as $i=1,2,5,6$, and hence $C_{L(G)}(b)$ has dimension at most 36 . But $C_{L(G)}(a)$ has dimension at least $\operatorname{dim} A_{6}=48$, a contradiction. In the last case $C_{V_{D_{6}}\left(\lambda_{i}\right)}(b)$ has 
dimension $0,14,8,0$ according as $i=1,2,5,6$, so $\operatorname{dim} C_{L(G)}(b) \leq 36$, again a contradiction.

Similar contradictions are reached when $S<A_{6}$ or $A_{1} D_{5}$ : for the $A_{6}$ case we obtain a group $\bar{S}$ contradicting $(\dagger)$ (note that the irreducible embedding 6 is not possible as its image in $A_{6}$ is $\left.P S L_{2}(q)\right)$; and for the $A_{1} D_{5}$ case we obtain $\bar{S}$ unless the projection of $S$ into the $D_{5}$ factor is $8 / 0$, in which case we obtain a contradiction by considering $C_{L(G)}(b)$ as above.

Lemma 5.5. Theorem 1 holds if $A=L_{3}(13)$ or $U_{3}(11)$.

Proof. Pick $a \in A$ of order 4 such that $C_{A}(a)^{(\infty)}=S \cong S L_{2}(p)$. Then $C_{G}(a)$ is one of the following groups (cf. [CG, Table 4]):

$$
T_{1} E_{7}, T_{1} A_{1} E_{6}, T_{1} D_{7}, T_{1} A_{7}, T_{1} A_{1} D_{6}, A_{1} A_{7}, A_{3} D_{5} .
$$

We now argue in a series of steps.

Step 1. If $C_{G}(a)=T_{1} E_{7}$, then the composition factors of $L(G) \downarrow A$ are among the modules $V_{A}(a b)$ with $a b \in\{00,11,30,03\}$.

To see this, observe first that the weights of $T_{1}$ on $L(G)$ are $2,1,0,-1,-2$, and $2,-2$ occur with multiplicity 1 ; these are the only weights which agree on $a$, and the sum of the two corresponding weight spaces is the -1-eigenspace for $a$. It follows that any $a$-invariant subspace of $L(G)$ containing this eigenspace is also $T_{1}$-invariant, and so is any $a$-invariant space which intersects the eigenspace in 0 only. In view of $(\dagger)$, it follows that some composition factor of $L(G) \downarrow A$ has -1-eigenspace of dimension 1.

Suppose $W=V_{A}(c d)=V_{A}(\lambda)$ is a composition factor on which $a$ has no eigenvalues -1 . We may assume the composition factor is restricted. Note that $Z(A)=1$, so $c \equiv d \bmod 3$. Let $\alpha, \beta$ be fundamental roots of an $A_{2}$ root system. We may assume that $c \geq d, \alpha(a)=i$ and $\beta(a)=1$. We know that $\lambda(a)=1, i$ or $-i$. If $\lambda(a)=1$ then $\lambda-2 \alpha-b \beta$ cannot be a weight of $W$ for any $b \geq 0$, so $c \leq 1$ and $d \leq 1$. This forces $c=d=0$. If $\lambda(a)=i$ then $\lambda-3 \alpha-b \beta$ cannot be a weight, so $c \leq 2, d \leq 2$, forcing $c=d=1$. Finally, if $\lambda(a)=-i$ then $\lambda-\alpha-b \beta$ cannot be a weight, forcing $c=d=0$, a contradiction.

Similarly, we find that the only possible composition factors on which $a$ has exactly one eigenvalue -1 are $V_{A}(30)$ and $V_{A}(03)$. This proves Step 1.

Step 2. $C_{G}(a) \neq T_{1} E_{7}$.

For suppose $C_{G}(a)=T_{1} E_{7}$. Then by Step 1, the highest weight of $S$ on $L(G)$ is 3. This is less than $(p-1) / 3$, and one now checks that the argument of [ST1, Lemma 2] (second paragraph of proof) provides a rank 1 torus of $G$ which fixes the same subspaces of $L(G)$ as some element of $S$ of order $p-1$, contrary to $(\dagger)$.

Step 3. $C_{G}(a) \neq T_{1} D_{7}$.

Suppose $C_{G}(a)=T_{1} D_{7}$, so that $S<D_{7}$. Observe first that by [LS2, Table 8.1],

$$
L(G) \downarrow D_{7}=\lambda_{2} / \lambda_{1}^{2} / \lambda_{6} / \lambda_{7} / 0 .
$$

Arguing as in 4.2 and 4.3 , we see that either $S<\bar{S}<D_{7}$ with $\bar{S} \cong S L_{2}(K)$ and all composition factors of $L(G) \downarrow \bar{S}$ of high weight less than $p$ (in which case (†) is violated), or the embedding of $S$ in $D_{7}$ is one of

$$
12 / 0,10 / 0^{3}, 8 / 4,8 / 2 / 0^{2}, 8 / 1^{2} / 0,5 / 5 / 0^{2}(p=11)
$$


(where as usual we specify the embedding by giving the composition factors of $S$ on the natural 14-dimensional module for $D_{7}$ ). Note that the embeddings $6 / 6$ and $8 / 0^{5}$ are not possible, as these correspond to a subgroup $P S L_{2}(q)$ of $D_{7}$ rather than $S L_{2}(q)$.

In the ensuing arguments, observe that since $A=L_{3}(13)$ or $U_{3}(11)$, every composition factor of $L(G) \downarrow A$ is of the form $V_{A}(a b)$ with $a \equiv b \bmod 3$.

If the embedding is $12 / 0$ then $p=13$ and, using the restriction $L(G) \downarrow D_{7}$ given above, together with [LS2, 2.13], we see that $L(G) \downarrow S$ has three composition factors with high weight 12 . Then by Proposition $1.9, L(G) \downarrow A$ must have three composition factors among $V_{A}(12,0), V_{A}(93), V_{A}(66)$. This forces $\operatorname{dim} L(G)>248$, a contradiction.

If the embedding is $10 / 0^{3}$, then $L(G) \downarrow S$ has at least five composition factors with high weight 10 , and again we obtain a contradiction using Proposition 1.9. If the embedding is $8 / 4$ then for $p=13$ we find that $L(G) \downarrow S=12^{1} / 11^{4} / \ldots$, and for $p=11, L(G) \downarrow S=10^{2} / 9^{4} / 8^{4} / 7^{6} / \ldots$, both of which conflict with 1.9 . If the embedding is $8 / 2 / 0^{2}$ or $8 / 1^{2} / 0$, we find that $L(G) \downarrow S=11^{4} / \ldots$ or $11^{2} / 10^{6} / \ldots$ (respectively) if $p=13$, and is $10^{2} / 9^{8} / \ldots$ or $10^{5} / 9^{6} / 8^{3} / \ldots$ if $p=11$; all these possibilities conflict with 1.9 .

Finally, the embedding $5 / 5 / 0^{2}$ is excluded exactly as in the proof of 5.3. This completes Step 3.

Step 4. $C_{G}(a) \neq T_{1} A_{7}, T_{1} A_{1} D_{6}, A_{1} A_{7}$ or $A_{3} D_{5}$.

The proof of this is entirely similar to that of Step 3: supposing $C_{G}(a)$ is one of the above, we find that either $S<\bar{S}<C_{G}(a)^{\prime}$ with $\bar{S}$ having all weights less than $p$, or the embedding of $S$ in $C_{G}(a)^{\prime}$ is one of the following:

\begin{tabular}{ll}
$C_{G}(a)^{\prime}$ & embedding $S<C_{G}(a)^{\prime}$ \\
\hline$A_{7}$ & 7 \\
$A_{1} A_{7}$ & 1,7 or $1,6 / 0(p=11)$ \\
$A_{1} D_{6}$ & $1,10 / 0$ or $1,8 / 2$ or $1,8 / 0^{3}$ or $1,5 / 5(p=11)$ \\
$A_{3} D_{5}$ & $3,8 / 0$
\end{tabular}

(note that in the last two rows we have assumed that $S \not \leq D_{7}$, in view of the argument for Step 3). For each of the above embeddings, it is straightforward to work out the composition factors of $L(G) \downarrow S$, and in all cases we find as in Step 3 that these conflict with 1.9 .

Step 5. $C_{G}(a) \neq T_{1} A_{1} E_{6}$.

The argument for this step is similar to the proof of 4.7. Let $S_{0}$ be the projection of $S$ to the factor $E_{6}$, pick $u \in S$ of order $p$, and write $u=u_{1} u_{0}$ with $u_{1} \in A_{1}$, $u_{0} \in S_{0}<E_{6}$. Note that $S_{0} \cong L_{2}(p)$.

Assume first that $u_{0}$ is a regular or semiregular element of $E_{6}$. As in the proof of 4.7, [ST2] then implies that $S_{0}<\bar{S}<E_{6}$, where $\bar{S} \cong A_{1}(K)$; and by [Se2, p.65] and [LS2, 2.5(ii)] we have, writing $V_{27}=V_{E_{6}}\left(\lambda_{1}\right)$, either

(i) $p=13, L\left(E_{6}\right) \downarrow \bar{S}=W(22) / W(16) / W(14) / W(10) / W(8) / W(2)$ and $V_{27} \downarrow \bar{S}$ $=W(16) / W(8) / 0$ (recall $W(r)$ denotes the Weyl module of high weigth $r$ ), or

(ii) $L\left(E_{6}\right) \downarrow \bar{S}=W(16) / W(14) / W(10)^{2} / W(8) / W(6) / W(4) / W(2)$ and $V_{27} \downarrow \bar{S}$ $=W(12) / W(8) / W(4)$. 
Moreover, by [LS2, §2],

$$
L(G) \downarrow A_{1} E_{6}=L\left(A_{1} E_{6}\right) / 1 \otimes \lambda_{1} / 1 \otimes \lambda_{6} / 0 \otimes \lambda_{1} / 0 \otimes \lambda_{6} /(1 \otimes 0)^{2} / 0 .
$$

Note that $S$ projects nontrivially into the $A_{1}$ factor (since the image of $S$ in the $E_{6}$ factor is $\left.P S L_{2}(q)\right)$. In case (i), we deduce that $L(G) \downarrow S$ has at least 3 composition factors with high weight 10 , and none with high weight 12 . Using 1.9 we find that this leads to a contradiction. In case (ii) with $p=13$, we have $L(G) \downarrow$ $S=12^{2} / 11^{4} / 10^{3} / 9^{2} / \ldots ;$ and in case (ii) with $p=11$, we have $L(G) \downarrow S=$ $10^{2} / 9^{4} / 8^{5} / \ldots$. Both of these restrictions conflict with the possibilities given by 1.9 .

Therefore $u_{0}$ is not regular or semiregular in $E_{6}$, so, as in the proof of 4.7, it lies in a subsystem subgroup $Y=D_{5}, A_{1} A_{5}$ or $A_{2} A_{2} A_{2}$. Using 1.7 for the $D_{5}$ case, we see that there is a subgroup $\bar{S} \cong A_{1}(K)$ of $Y$ containing $u_{0}$ and having restricted composition factors on the usual modules for all factors of $Y$. The restrictions $V_{27} \downarrow Y$ are given by [LS2, Table 8.7], and using [LS2, 2.13] we deduce that all composition factors of $V_{27} \downarrow \bar{S}$ are restricted. Therefore, if $U$ is a connected unipotent subgroup of $\bar{S}$ containing $u_{0}$, then by [LST, 1.14], $u_{0}$ and $U$ fix the same subspaces of $V_{27}$. Now $S_{0}=A_{1}(p)$ is not irreducible on $V_{27}$; hence neither is $\left\langle S_{0}, U\right\rangle$. Thus $\left\langle S_{0}, U\right\rangle^{0}$ is a proper nontrivial connected subgroup of $E_{6}$, hence lies in a maximal connected subgroup, $M$ say. The possibilities for $M$ are given by [Se2, Theorem 1].

Suppose $M$ contains a maximal torus of $E_{6}$. If $M$ is reductive, then $M^{\prime}$ is a subsystem subgroup, hence lies in a subgroup $D_{5}, A_{1} A_{5}$ or $A_{2} A_{2} A_{2}$. We find in the usual way that either $S$ lies in a subgroup $A_{1}(K)$ having all composition factors on $L(G)$ of high weight less than $p$, or $S_{0}<D_{5}$ with embedding 8/0. But in this case $S<A_{1} D_{5}<D_{7}$, which was excluded in Step 3.

Now suppose $M$ is parabolic. Choose a minimal parabolic $P=Q L$ of $E_{6}$ containing $S_{0}$, with unipotent radical $Q$ and Levi subgroup $L$. The $L$-composition factors of $Q$ have the structure of $K L$-modules, with high weights given by [LS2, 3.1]. Using 1.2, we find that either $H^{1}\left(S_{0}, V\right)=0$ for all such modules $V$, or $L=D_{5}$ and the embedding of $S_{0}$ in $L$ modulo $Q$ is 8/0. In the latter case, $S<Q A_{1} D_{5}$, so $S$ has the same composition factors on $L(G)$ as a suitable subgroup $A_{1}(p)$ of $A_{1} D_{5}<D_{7}$, and the proof of Step 3 gives a contradiction. And in the former case, $S_{0}$ lies in a conjugate of $L$, a case dealt with in the previous paragraph.

Thus $M$ does not contain a maximal torus of $E_{6}$. The argument of the previous two paragraphs shows that $S_{0}$ lies in no subgroup of maximal rank in $E_{6}$. Then by [Se2], $M=A_{2}, G_{2}, F_{4}$ or $A_{2} G_{2}$; the restrictions of $L\left(E_{6}\right)$ and $V_{27}$ to each of these subgroups are given in [LS2, §2]. The fact that $S_{0}$ lies in no maximal rank subgroup of $E_{6}$ implies that $S_{0}$ also lies in no maximal rank subgroup of $M$ (since otherwise $S_{0}$ would centralize a nontrivial semisimple element $t$ in the centre of a maximal rank subgroup of $M$, hence lie in the maximal rank subgroup $C_{E_{6}}(t)$ ).

Write $R=\left\langle S_{0}, U\right\rangle$. Observe first that $R$ does not act irreducibly in dimension 27 or 26 , so $R$ is not equal to one of the maximal subgroups $G_{2}, A_{2}$ or $F_{4}$ of $E_{6}$. Also, if $R$ lies in a maximal $G_{2}$, then by [Se2, pp.193 and 65], $R=A_{1}$ and $S_{0}<R$ has composition factors as in (ii) above, giving a contradiction as before. And if $R$ lies in a maximal $A_{2}$, then $R=A_{1}$ has all composition factors on $L(G)$ of high weight less than $p$.

Now suppose $R$ lies in a maximal subgroup $A_{2} G_{2}$ of $E_{6}$. Then $S_{0}<A_{2} G_{2}$. Let $S_{1}$ be the projection of $S_{0}$ in the factor $G_{2}$. Then $S_{1}=L_{2}(p)<G_{2}<B_{3}$. There is a subgroup $\bar{S}_{1} \cong A_{1}(K)$ of $B_{3}$ containing $S_{1}$, with all weights on $L\left(B_{3}\right)$ less than $p$. 
Hence by $1.5, \bar{S}_{1}$ fixes all $S_{1}$-invariant subspaces of $L\left(B_{3}\right)$, and in particular fixes $L\left(G_{2}\right)$. Therefore $S_{1}<\bar{S}_{1}<G_{2}$; as $S_{1}$ is not contained in a proper maximal rank subgroup of $G_{2}$, we have $V_{G_{2}}\left(\lambda_{1}\right) \downarrow \bar{S}_{1}=6$ and $L\left(G_{2}\right) \downarrow \bar{S}_{1}=10 / 2$. It follows that $S<\bar{S}<A_{1} A_{2} G_{2}$ with $\bar{S} \cong A_{1}(K)$ having all weights on $L(G)$ less than $p$.

Finally, suppose $R$ lies in a maximal subgroup $F_{4}$ of $E_{6}$. By [Se2] again, $R$ lies in a maximal subgroup $A_{1}$ or $A_{1} G_{2}$ of this $F_{4}$. The subgroup $A_{1} G_{2}$ lies in a maximal $A_{2} G_{2}$ of $E_{6}$, a case handled in the previous paragraph. Hence $R=A_{1}$; now $R$ has composition factors as in (i) above, giving a contradiction as before.

This completes the proof of Step 5, and hence of the lemma.

Lemmas 5.2-5.5, together with the remarks at the beginning of this section, complete the proof of Theorem 1 for $p \neq 2$.

\section{Proof of Theorem 1 for $p=2$}

In this section we complete the proof of Theorem 1 by handling the case where $p=2$. Thus we assume that $X=X(q)<G$ with $q=2^{e} \geq 16$, and $G=E_{8}$ (as we may assume by 3.1). As before, by Proposition 2 we can take it that $X \neq L_{2}(q)$ or ${ }^{2} B_{2}(q)$. Moreover, $X \neq A_{2}^{\epsilon}(16)$ by the hypothesis of Theorem 1.

Observe that $X$ contains a subgroup $A$, where $A=A_{2}^{\epsilon}(q)$ or $B_{2}(q)$ if $q \geq 32$, and $A=B_{2}(16)$ or $G_{2}(16)$ if $q=16$. Pick $a \in A$ of order divisible by $(q-\epsilon) /(3, q-\epsilon)$ if $A=A_{2}^{\epsilon}(q)$, and of order $q+1$ otherwise, such that $C_{A}(a)^{(\infty)}=S \cong S L_{2}(q)$, generated by long root subgroups of $A$. Note that either $o(a) \geq 17$ or $o(a)=11$ (which occurs only if $A=U_{3}(32)$.

In view of 3.2 , we continue to adopt the assumption $(\dagger)$ made immediately after 3.2 .

We begin with several preliminary results which will be required in the proof.

Lemma 6.1. The restrictions to $S$ of $V_{A}(10), V_{A}(01)$ and $V_{A}(11)$ are as in Table 4.

\section{TABLE 4}

\begin{tabular}{lllll}
$A$ & $V_{A}(10) \downarrow S$ & $V_{A}(01) \downarrow S$ & $V_{A}(11) \downarrow S$ & $\operatorname{dim} V_{A}(10), V_{A}(01), V_{A}(11)$ \\
\hline$A_{2}^{\epsilon}(q)$ & $1 / 0$ & $1 / 0$ & $1^{2} / 1^{(2)} / 0^{2}$ & $3,3,8$ \\
$B_{2}(q)$ & $1^{2}$ & $1 / 0^{2}$ & $1^{4} /\left(1^{(2)}\right)^{2} / 0^{4}$ & $4,4,16$ \\
$G_{2}(q)$ & $1^{2} / 0^{2}$ & $1^{4} / 1^{(2)} / 0^{4}$ & $\left(1 \otimes 1^{(2)}\right)^{2} / 1^{10} /$ & $6,14,64$ \\
& & & $\left(1^{(2)}\right)^{9} / 0^{18}$ & \\
\hline
\end{tabular}

Proof. This is elementary for the high weights 10 and 01, and follows for 11 from the following facts: for $A_{2}$, the module 11 is the quotient of $10 \otimes 01$ by a trivial submodule 00 ; for $B_{2}, 11=10 \otimes 01$; and for $G_{2}, 11$ is the quotient of $10 \otimes 01$ by a submodule $10 \oplus 01$.

Lemma 6.2. (i) If $L(G) \downarrow S$ has a composition factor $1^{\left(2^{i_{1}}\right)} \otimes 1^{\left(2^{i_{2}}\right)} \otimes 1^{\left(2^{i_{3}}\right)} \otimes$ $1^{\left(2^{i_{4}}\right)}$, then $A \neq B_{2}(q)$ or $G_{2}(q)$; moreover, if this composition factor occurs with multiplicity 1 , then $A \neq A_{2}^{\epsilon}(q)$ also.

(ii) If $L(G) \downarrow S$ has a composition factor $1^{\left(2^{i^{1}}\right)} \otimes \ldots \otimes 1^{\left(2^{i_{r}}\right)}$ with $r=3$ or 4 , and has no composition factor $1^{\left(2^{i_{1}}\right)} \otimes \ldots \otimes 1^{\left(2^{i_{r}-1}\right)}$, then $A \neq A_{2}^{\epsilon}(q)$ or $G_{2}(q)$. 
(iii) If $L(G) \downarrow S$ has a composition factor $1^{\left(2^{i_{1}}\right)} \otimes 1^{\left(2^{i_{2}}\right)} \otimes 1^{\left(2^{i_{3}}\right)}$ with multiplicity 1 , then it has $1^{\left(2^{i_{1}}\right)} \otimes 1^{\left(2^{i_{2}}\right)}$ as a composition factor also.

(iv) If $L(G) \downarrow S$ has a composition factor $1^{\left(2^{i_{1}}\right)} \otimes 1^{\left(2^{i_{2}}\right)} \otimes 1^{\left(2^{i_{3}}\right)}$ with multiplicity less than 8 , or has composition factors $1^{\left(2^{i_{1}}\right)} \otimes 1^{\left(2^{i_{2}}\right)} \otimes 1^{\left(2^{i_{3}}\right)}, 1^{\left(2^{i_{1}}\right)} \otimes 1^{\left(2^{i_{2}}\right)}$, the latter with multiplicity less than 8 , or has two different composition factors $1^{\left(2^{i_{1}}\right)} \otimes$ $1^{\left(2^{i_{2}}\right)} \otimes 1^{\left(2^{i_{3}}\right)}$ and $1^{\left(2^{j_{1}}\right)} \otimes 1^{\left(2^{j_{2}}\right)} \otimes 1^{\left(2^{j_{3}}\right)}$, then $A \neq G_{2}(q)$.

(v) If $L(G) \downarrow S$ has composition factors $1^{\left(2^{i_{1}}\right)} \otimes 1^{\left(2^{i_{2}}\right)} \otimes 1^{\left(2^{i_{3}}\right)}$ with multiplicity 2 , $1^{\left(2^{i_{1}}\right)} \otimes 1^{\left(2^{i_{2}}\right)}$ with multiplicity less than 4 , and no composition factor $1^{\left(2^{i_{2}}\right)} \otimes 1^{\left(2^{i_{3}}\right)}$, then $A \neq B_{2}(q)$.

(vi) If $L(G) \downarrow S$ has composition factors $1^{\left(2^{i_{1}}\right)} \otimes 1^{\left(2^{i_{2}}\right)} \otimes 1^{\left(2^{i_{3}}\right)}$ with multiplicity a, $1^{\left(2^{i_{1}}\right)} \otimes 1^{\left(2^{i_{2}}\right)} \otimes 1^{\left(2^{j}\right)}\left(j \neq i_{3}\right)$ with multiplicity $b$, and $1^{\left(2^{i_{1}}\right)} \otimes 1^{\left(2^{i_{2}}\right)}$ with multiplicity less than $a+b$, then $A \neq A_{2}^{\epsilon}(q)$.

Proof. (i) Note first that $L(G) \downarrow S$ has no $r$-fold tensor composition factors with $r \geq 5$, since $\operatorname{dim} L(G)<3^{5}$. By $6.1, L(G) \downarrow A$ must also have a 4 -fold tensor composition factor. If $A=B_{2}(q)$ or $G_{2}(q)$, this has dimension at least $4^{4}$ or $6^{4}$, which is greater than 248, a contradiction; and if $A=A_{2}^{\epsilon}(q)$ and the 4-fold factor in $L(G) \downarrow S$ occurs with multiplicity 1 , then $L(G) \downarrow A$ has a self-dual 4 -fold factor, of dimension at least $8^{4}$, again a contradiction.

(ii) Suppose $A=A_{2}^{\epsilon}(q)$ or $G_{2}(q)$. By 6.1 and dimensions, $L(G) \downarrow A$ must have a composition factor $10^{\left(2^{i_{1}}\right)} \otimes \ldots \otimes 10^{\left(2^{i_{r}}\right)}$ (for type $A_{2}$, each 10 could also be 01); however the restriction of this to $S$ has a composition factor $1^{\left(2^{i_{1}}\right)} \otimes \ldots \otimes 1^{\left(2^{i} r-1\right)}$, contrary to the hypothesis of (ii).

(iii) This follows from (ii) unless $A=B_{2}(q)$. For this case, by 6.1 , the multiplicity 1 assumption forces $L(G) \downarrow A$ to have a composition factor $01^{\left(2^{i_{1}}\right)} \otimes 01^{\left(2^{i_{2}}\right)} \otimes 01^{\left(2^{i_{3}}\right)}$; however, the restriction of this to $S$ has a composition factor $1^{\left(2^{i_{1}}\right)} \otimes 1^{\left(2^{i_{2}}\right)}$.

(iv) Suppose $A=G_{2}(q)$. The hypothesis implies that $L(G) \downarrow A$ has a 3 -fold composition factor, which by dimensions must be $10^{\left(2^{i_{1}}\right)} \otimes 10^{\left(2^{i_{2}}\right)} \otimes 10^{\left(2^{i_{3}}\right)}$. The restriction of this to $S$ conflicts with the assumptions in (iv).

(v) Suppose $A=B_{2}(q)$. Again $L(G) \downarrow A$ must have a 3 -fold composition factor $\mu_{1}^{\left(2^{i_{1}}\right)} \otimes \mu_{2}^{\left(2^{i_{2}}\right)} \otimes \mu_{3}^{\left(2^{i_{3}}\right)}$, where each $\mu_{i} \in\{10,01\}$. Each possibility for the $\mu_{i}$ conflicts with the assumptions on multiplicities.

(vi) This follows from an easy extension of the argument for (ii).

Lemma 6.3. Let $e \geq 5$, and take $\mathbb{Z}_{e}=\{0,1, \ldots, e-1\}$ to act additively on itself (i.e. $a \in \mathbb{Z}_{e}$ sends $\left.x \rightarrow x+a\right)$. Let $k \in\{3,4,5\}$, and let $i_{1} \ldots i_{k}$ be an unordered $k$-tuple of elements of $\mathbb{Z}_{e}$. Then $i_{1} \ldots i_{k}$ is in the $\mathbb{Z}_{e}$-orbit of a $k$-tuple $j_{1} \ldots j_{k}$ satisfying one of the following conditions:

(i) $j_{r} \leq e-3$ for all $r$;

(ii) taking $j_{1} \leq \ldots \leq j_{k}$, we have $j_{k}=e-2, j_{k-1} \leq e-3$, and, if $j_{k-1}=e-3$, then $j_{k-2} \leq e-\overline{4}$;

(iii) $e=k=5$ and $j_{1} \ldots j_{k}=01234,01223,00223,01133$ or 01233 .

Proof. Assume that (i) and (ii) fail. If $e=k=5$ and all the $i_{j}$ are distinct, then clearly $i_{1} \ldots i_{k}=01234$, as in (iii). So suppose this is not the case. Then the number of distinct $i_{j}$ is less than $e$. Take $i_{1} \leq \ldots \leq i_{k}$. Applying an element of $\mathbb{Z}_{e}$, we can assume that $i_{k}<e-1$. Therefore as (i) fails, we have $i_{k}=e-2$; we also have $i_{1}=0$, since otherwise we can apply the element -1 of $\mathbb{Z}_{e}$ to obtain conclusion (i). 
Thus we have $i_{1}=0, i_{k}=e-2$. Applying the element 2 of $\mathbb{Z}_{e}$, we obtain (i) again, unless some $i_{j}$ is $e-3$ or $e-4$. As (ii) fails, we deduce that either

(a) $i_{1} \ldots i_{k}$ contains $0, e-3, e-3, e-2$, or

(b) $i_{1} \ldots i_{k}$ contains $0, e-2, e-2$.

Consider case (a). If $k \leq 4$, application of the element 3 of $\mathbb{Z}_{e}$ gives the tuple 3001 , which satisfies (ii). Now suppose $k=5$, so the tuple is $0, i, e-3, e-3, e-2$. Applying $3 \in \mathbb{Z}_{e}$ gives the tuple $3, i+3,0,0,1$; this satisfies (i) or (ii) unless either $i=e-4$ or $e=5, i=0$. In the latter case the tuple is 33001, in the same orbit as 00223, as in (iii). And if $i=e-4$ the tuple is in the orbit of 40112. As (ii) fails, $e=5$; then this is in the orbit of 01223, under (iii).

Now consider case (b). If $k \leq 4$ the tuple is $0, i, e-2, e-2$, which is in the same $\mathbb{Z}_{e^{- \text {orbit }}}$ as $2, i+2,0,0$; this satisfies (ii) unless $i=e-3$, in which case the tuple is in the orbit of 3011, which satisfies (ii).

Now suppose $k=5$, so the tuple is $0, i, j, e-2, e-2(i \leq j)$, in the same orbit as $2, i+2, j+2,0,0$. As (ii) fails, either $j=e-3$, or $i=j=e-4$, or $e=5, i=0, j=1$. In the last case the tuple is 00223, under (iii). If $i=j=e-4$ the tuple is in the orbit of 40022; as (i),(ii) fail, we must have $e=5$ here, in which case the tuple is in the orbit of 01133 , under (iii). Finally, suppose $j=e-3$. Then the tuple is in the orbit of $3, i+3,0,1,1$; as (ii) fails, either $i=e-4$ or $e=5, i=0$. In the latter case the tuple is 01133 , under (iii). In the former, the tuple is in the orbit of 40122; as (ii) fails, $e=5$ and this is in the orbit of 01233 , under (iii).

Corollary 6.4. Let $e \geq 5, k \in\{3,4\}$, and let $i_{1} \ldots i_{k}$ be an unordered $k$-tuple of elements of $\mathbb{Z}_{e}$; if $k=4$, assume that $i_{1}, \ldots, i_{k}$ are not all distinct. Then $i_{1} \ldots i_{k}$ is in the $\mathbb{Z}_{e}$-orbit of a $k$-tuple $j_{1} \ldots j_{k}$ satisfying one of the following conditions:

(i) $j_{r} \leq e-3$ for all $r$;

(ii) $e=5$ and $j_{1} \ldots j_{k}=0013,0023,0113$ or 013 ;

(iii) $e=6$ and $j_{1} \ldots j_{k}=0024$ or 024 .

Proof. Consider first the case where $k=4$, and take $i_{1} \leq \ldots \leq i_{4}$. As in the proof of the previous result, we may take $i_{4}=e-2, i_{1}=0$. By 6.3 we may assume that 6.3 (ii) holds. Thus $i_{1} \ldots i_{4}$ is either $0, i, e-3, e-2$ (with $i \leq e-4$ ) or $0, i, j, e-2$ (with $i \leq j \leq e-4$ ). In the first case the tuple is in the orbit of $3, i+3,0,1$. This satisfies (i) unless $i=e-4$ or $e=5, i=0$; in the latter the tuple is in the orbit of 0023, under (iii), and in the former, the tuple has 4 distinct elements, contrary to hypothesis.

Now suppose the tuple is $0, i, j, e-2$ with $i \leq j \leq e-4$. This is in the orbit of $2, i+2, j+2,0$, which satisfies (i) unless $j=e-4$. Since $i_{1}, \ldots i_{4}$ are not all distinct, $i$ must be 0 or $e-4$. Hence the tuple is in the orbit of 0244 or 0024 . These satisfy (i) unless $e=5$ or 6 , in which case they lie in the orbits of tuples under (iii). This completes the case $k=4$, and the case $k=3$ is entirely similar.

As in previous sections, in the course of our proof of Theorem 1 we shall study the embedding of our subgroup $S \cong S L_{2}(q)$ in $C_{G}(a)$. As before, define $D=C_{G}(a)^{\prime}$, a connected semisimple group. By Lemmas 3.4-3.7 we know that $D$ is as in 3.5(5). In particular, either $D$ has a factor $D_{4}$ or $D_{5}$, or $D$ is a product of factors $A_{r}$. For the $D_{4}, D_{5}$ possibilities we need to study in some detail the embeddings of subgroups $S_{2}(q)$ in these groups.

For this purpose we now introduce some notation for certain connected semisimple subgroups of $D_{4}, D_{5}$ (in characteristic 2). It is convenient to treat these groups 
in the forms $S O_{8}, S O_{10}$. First, we denote by $A_{1}$ a connected fundamental subgroup $S L_{2}$ in $S O_{8}$ or $S O_{10}$. Thus $A_{1}^{4}$ denotes a subgroup $S_{4} S_{4}$ in $S_{8}$, naturally embedded. Taking a subgroup $\mathrm{SO}_{4}$ of $\mathrm{SO}_{8}$ fixing pointwise a non-degenerate 4-space, we write $B_{1}$ for a subgroup $S_{3}$ of this $S_{4}$ fixing a nonsingular vector. Then $S O_{6}$ contains a subgroup $B_{1}^{2}, S O_{8}$ a subgroup $B_{1}^{3}$, and $S O_{10}$ a subgroup $B_{1}^{4}$; in each case, the subgroup $B_{1}^{k}$ fixes a unique nonsingular 1-space. Note that if $v$ is a nonsingular vector then $\left(\mathrm{SO}_{10}\right)_{v}=\mathrm{SO}_{9}$, and a surjective morphism $\mathrm{SO}_{9} \rightarrow \mathrm{Sp}_{8}$ sends $B_{1}^{4} \rightarrow C_{1}^{4}=\left(S p_{2}\right)^{4}$, naturally embedded in $S p_{8}$. Finally, $S O_{8}$ contains a subgroup $S p_{2} \otimes S p_{2} \otimes S p_{2}$, which we denote by $C_{1}^{3}$; and $C_{1}^{2}$ denotes a subgroup $S p_{2} \otimes S p_{2}$ of this.

Let $S_{0}=A_{1}\left(2^{e}\right)$, and fix an injection $\iota: S_{0} \rightarrow S L_{2}(K)$. Whenever $S_{0}=$ $A_{1}\left(2^{e}\right)$ maps homomorphically into a commuting product $A_{1}^{k} B_{1}^{l}$, up to conjugacy the projections of $S_{0}$ to the factors are either trivial, or are field twists of the injection $\iota$. In the following result, when we say $S_{0}$ has "distinct twists on the $B_{1}$ factors", we mean that the twists involved in all the nontrivial projections to $B_{1}$ factors are all distinct.

Finally, if $Y$ is a connected reductive group, we shall denote by $Q Y$ a connected group with unipotent radical $Q$.

Lemma 6.5. Let $S_{0}=S L_{2}\left(2^{e}\right)(e \geq 4)$, and suppose $S_{0}<D_{r}$ with $r=4$ or 5 .

(i) If $r=4$ then $S_{0}$ lies in one of the following connected subgroups of $D_{4}$ :

$$
A_{1}^{4}, Q A_{1}^{3}, B_{1}^{3}, Q B_{1}^{2}, Q C_{1}^{2}, C_{1}^{3} \text {. }
$$

In the last four cases $S_{0}$ has distinct twists on the $B_{1}$ and $C_{1}$ factors.

(ii) If $r=5$ then $S_{0}$ lies in one of the following connected subgroups of $D_{5}$ :

$$
Q A_{1}^{4}, B_{1}^{4}, A_{1}^{2} B_{1}^{2}, Q A_{1} B_{1}^{2}, Q B_{1}^{3}, Q C_{1}^{2}, C_{1}^{3} \text {. }
$$

In the third case $A_{1}^{2} B_{1}^{2}<S O_{4} S O_{6}$. Also, $S_{0}$ has distinct twists on the $B_{1}$ and $C_{1}$ factors in all cases with such factors.

Proof. In the course of the proof we shall require information on the possible embeddings of $S_{0}$ in the symplectic groups $S p_{4}, S p_{6}, S p_{8}$, as well as in $S O_{6}, S O_{8}, S O_{10}$. It is convenient to start with the smallest dimensions and work our way upwards. For a classical group $G=S p_{2 n}$ or $S O_{2 n}$, we shall denote the natural $G$-module by $V_{2 n}$. If $S_{0}<G$, let $W$ be an irreducible $S_{0}$-submodule of $V_{2 n}$; then $W$ has dimension $1,2,4$ or 8 , and $W$ is either non-degenerate or totally singular, or, when $G$ is orthogonal, a nonsingular 1-space.

Suppose that $S_{0}<S p_{4}$, with natural module $V_{4}$. As above, let $W$ be an irreducible $S_{0}$-submodule of $V_{4}$. If $W=V_{4}$ then $V_{4} \downarrow S_{0}=1^{\left(2^{i_{1}}\right)} \otimes 1^{\left(2^{i_{2}}\right)}$, and $S_{0}$ lies in a connected subgroup $S p_{2} \otimes S p_{2}=C_{1} \otimes C_{1}$ of $S p_{4}$. If $W$ is a non-degenerate 2-space then $S_{0}<S p_{2} \perp S p_{2}=C_{1} C_{1}$. If $W$ is a 1 -space, then $V_{4} \downarrow S_{0}=0 / 1^{\left(2^{i}\right)} / 0$; we can take this to be indecomposable, as otherwise we are in the previous case. Here $S_{0}$ lies in a parabolic subgroup $Q C_{1} T_{1}$ of $S p_{4}$, and the unipotent radical $Q$ has a 1dimensional subgroup $Z$ such that $Q / Z$ is an $S_{0}$-module of high weight 1 ; moreover, $S_{0}$ acts trivially on $Z$. Choose $\tilde{S}_{0}<C_{1}$ such that $S_{0} \cong \tilde{S}_{0}$ and $Q S_{0}=Q \tilde{S}_{0}$. Then $H^{1}\left(\tilde{S}_{0}, Q / Z\right)$ is 1-dimensional by 1.2 , so under the action of $(Q / Z) T_{1}$ there are just 2 classes of complements to $Q / Z$ in $(Q / Z) \tilde{S}_{0}$, both of which give complements to $Q$ in $Q \tilde{S}_{0}$. One of these, $\tilde{S}_{0}$, lies in $C_{1}$; the other, $S_{0}$, lies in an indecomposable $B_{1}$ contained in $S_{4}<S p_{4}$. Consequently $S_{0}<B_{1}<C_{1} \otimes C_{1}=S O_{4}$ in this case. The last possibility is that $W$ is a singular 2-space. A graph automorphism $\tau$ of 
$S p_{4}$ maps the stabilizer of $W$ to the stabilizer of a 1-space. Hence by the previous case, $S_{0}^{\tau}$ lies in $C_{1} \otimes C_{1}$, whence $S_{0}$ lies in $C_{1} C_{1}$. Summarising, we have shown that if $S_{0}<S p_{4}$ then $S_{0}$ lies in either $C_{1} \otimes C_{1}$ or $C_{1} C_{1}$.

Next suppose $S_{0}<S O_{6}$, with natural module $V_{6}$. If $W$ is non-degenerate of dimension 2 or 4 , then $S_{0}<S O_{4}=A_{1}^{2}$. If $W$ is a singular 1-space or 2-space, then $S_{0}<Q A_{1}^{2}$ or $Q A_{1}$. The only other possibility is that $W$ is a nonsingular 1-space, in which case $V_{6} \downarrow S_{0}=0 / V_{4} / 0$, where $V_{4}$ is symplectic 4-space. Applying the previous paragraph, $S_{0}$ maps into a subgroup $C_{1} C_{1}$ or $C_{1} \otimes C_{1}$ of $S p\left(V_{4}\right)$. Thus $S_{0}$ lies in a subgroup $B_{1}^{2}$ or $A_{1}^{2}$ of $S O_{6}$. Moreover, if $S_{0}<B_{1}^{2}$ with equal twists on the factors, then $V_{6} \downarrow S_{0}=0 / 1^{\left(2^{i}\right)} / 1^{\left(2^{i}\right)} / 0$; as $\operatorname{Ext}_{S_{0}}^{1}\left(1^{\left(2^{i}\right)}, 0\right)$ is 1-dimensional by 1.2 , it follows that $S_{0}$ fixes a singular 2-space, yielding $S_{0}<Q A_{1}$. Summarising, if $S_{0}<S O_{6}$ then either $S_{0}<Q A_{1}^{2}$ or $S_{0}<B_{1}^{2}$ with distinct twists on the $B_{1}$ factors.

Next consider $S_{0}<S p_{6}$, with natural module $V_{6}$. If $W$ is non-degenerate then $S_{0}<C_{1} C_{2}$, so by the $S p_{4}$ case handled above, $S_{0}$ lies in $C_{1}\left(C_{1} \otimes C_{1}\right)$ or $C_{1}^{3}$. If $W$ is a singular 2-space then $S_{0}<Q A_{1} C_{1}$. Moreover, either $V_{6} \downarrow S_{0}=1^{\left(2^{i}\right)} / 1^{\left(2^{j}\right)} / 1^{\left(2^{i}\right)}$, completely reducible by 1.2 , or $V_{6} \downarrow S_{0}=1^{\left(2^{i}\right)} / 0^{2} / 1^{\left(2^{i}\right)}$; hence either $S_{0}<A_{1} C_{1}$ or $S_{0}<Q A_{1}$. Finally, if $W$ is a 1-space then $S_{0}<Q C_{2}$, hence $S_{0}$ lies in $Q\left(C_{1} \otimes C_{1}\right)$ or $Q C_{1} C_{1}$.

Similarly, if $S_{0}<S p_{8}$ then $S_{0}$ lies one of the subgroups $C_{1} \otimes C_{1} \otimes C_{1}$ (with $S_{0}$ irreducible on $V_{8}$ ), $C_{2} C_{2}, C_{1} C_{3}, Q A_{1} C_{2}, Q C_{3}$ or $Q A_{3}$ (in the last case $W$ is a singular 4-space), and we can apply the previous conclusions for subgroups of $C_{2}, C_{3}$ to refine this list.

Now we are ready to consider $S_{0}<S O_{8}$, with natural module $V_{8}$. If $W=V_{8}$ then $S_{0}<C_{1}^{3}$, with distinct twists on the factors, and if $W$ is a non-degenerate 4-space then $S_{0}<A_{1}^{4}$. If $W$ is a singular 4-space, then $W \downarrow S_{0}=1^{\left(2^{i}\right)} \otimes 1^{\left(2^{j}\right)}$ and $S_{0}<Q C_{1}^{2}<Q A_{3}$. If $W$ is a singular 2-space then $S_{0}<Q A_{1}^{3}$. And if $W$ is a singular 1-space, then $V_{8} \downarrow S_{0}=0 / V_{6} / 0$ with $V_{6}$ an $S O_{6}$-space, so by the $S O_{6}$ case above, $S_{0}$ lies in $Q A_{1}^{2}$ or $Q B_{1}^{2}$ (with distinct twists in the latter case).

To complete the analysis of $S_{0}<S O_{8}$, it remains to handle the case where $W$ is a nonsingular 1-space. Here $V_{8} \downarrow S_{0}=0 / V_{6} / 0$, where $V_{6}$ is symplectic 6space, and $S_{0}$ maps into $S p_{6}$. By the $S p_{6}$ case above, $S_{0}$ maps into a subgroup $C_{1}\left(C_{1} \otimes C_{1}\right), C_{1}^{3}, Q A_{1} C_{1}, Q\left(C_{1} \otimes C_{1}\right)$ or $Q C_{1} C_{1}$ of $S p_{6}$. In the first case $S_{0}<$ $B_{1} A_{1}^{2}<A_{1}^{4}$ (in $S O_{8}$ ). In the second, $S_{0}<B_{1}^{3}$; and if $S_{0}$ has equal twists on two of the $B_{1}$ factors here, then $V_{8} \downarrow S_{0}=0 / 1^{\left(2^{i}\right)} / 1^{\left(2^{i}\right)} / \ldots$, and an application of 1.2 shows that $S_{0}$ fixes a 2 -space $1^{\left(2^{i}\right)}$, putting us in a previous case. In the third case $S_{0}<Q A_{1} B_{1}$, which lies in $Q A_{1}^{3}$ in $S O_{8}$. In the fourth case $S_{0}<Q A_{1}^{2}$. Finally, in the last case $S_{0}<Q B_{1}^{2}$, and if $S_{0}$ has equal twists in the $B_{1}$ factors then $S_{0}<Q A_{1}$. This completes the proof of part (i) of the lemma.

Now consider $S_{0}<S O_{10}$, with natural module $V_{10}$. If $W$ has dimension 8 then $S_{0}<C_{1}^{3}$ (with distinct twists). If $W$ is a non-degenerate 4 -space then $S_{0}<$ $S O_{4} S O_{6}$, so by the $S O_{6}$ case $S_{0}$ lies in $Q A_{1}^{4}$ or $A_{1}^{2} B_{1}^{2}$. If $W$ is a singular 4-space then $V_{10} \downarrow S_{0}=\left(1^{\left(2^{i}\right)} \otimes 1^{\left(2^{j}\right)}\right)^{2} / 0^{2}$, and $S_{0}<Q C_{1}^{2}$. And if $W$ is a singular 1-space, then $S_{0}<Q S O_{8}$, and conclusion (ii) follows from the $S_{8}$ case (i) (note that the possibility $S_{0}<Q C_{1}^{3}$ only arises in the $S O_{8}$ proof when $S_{0}$ is irreducible on $V_{8}$, in which case $V_{10} \downarrow S_{0}$ is completely reducible by 1.2 , giving $S_{0}<C_{1}^{3}$ with distinct twists).

Next suppose that $W$ is a nonsingular 1 -space, so $V_{10} \downarrow S_{0}=0 / V_{8} / 0$, where $V_{8}$ is $S p_{8}$-space. By the $S p_{8}$ case above, $S_{0}$ maps into a subgroup $C_{1} \otimes C_{1} \otimes C_{1}$, 
$C_{2} C_{2}, C_{1} C_{3}, Q A_{1} C_{2}, Q A_{3}$ or $Q C_{3}$ of $S p_{8}$. In the first case we have $S_{0}<C_{1}^{3}$ with distinct twists. Next, if $S_{0}$ maps into $C_{2} C_{2}$ then, by the $S p_{4}$ case above, $S_{0}$ lies in $A_{1}^{4}, A_{1}^{2} B_{1}^{2}$ or $B_{1}^{4}$ in $S O_{10}$. Moreover we may take it that $S_{0}$ has distinct twists on the $B_{1}$ factors, since equal twists on a pair of $B_{1}$ factors allow us to replace the group $B_{1}^{2}$ by $Q A_{1}$, putting $S_{0}$ inside $Q A_{1}^{3}$ or $Q A_{1} B_{1}^{2}$, as in the conclusion of (ii). If $S_{0}$ maps into $C_{1} C_{3}$ then, by the $S p_{6}$ case, $S_{0}$ maps into $C_{1}^{2}\left(C_{1} \otimes C_{1}\right), C_{1}^{4}$, $C_{1}^{2} A_{1}, Q A_{1} C_{1}, Q C_{1}\left(C_{1} \otimes C_{1}\right)$, or $Q C_{1}^{3}$. Hence in $S O_{10}, S_{0}$ lies in $A_{1}^{2} B_{1}^{2}, B_{1}^{4}, A_{1} B_{1}^{2}$, $Q A_{1} B_{1}, Q A_{1}^{2} B_{1}$ or $Q B_{1}^{3}$. The only subgroup here not in conclusion (ii) is $Q A_{1}^{2} B_{1}$, which lies in $Q A_{1}^{4}$. Next, if $S_{0}$ maps into $Q A_{1} C_{2}$ in $S p_{8}$, then $S_{0}<Q A_{1}^{3}$ or $Q A_{1} B_{1}^{2}$ in $S O_{10}$. If $S_{0}<Q A_{3}$ then $S_{0}<Q C_{1}^{2}$. Finally in this case ( $W$ a nonsingular 1space), if $S_{0}$ maps into $Q C_{3}$ in $S p_{8}$, then $S_{0}$ fixes a singular 1-space in $V_{10}$, putting us in a previous case.

It remains to handle the case where $W$ is a singular 2-space. Here $S_{0}<Q A_{1} D_{3}$, so by the $S O_{6}$ case, $S_{0}$ lies in either $Q A_{1}^{3}$ or $Q A_{1} B_{1}^{2}$ (with distinct twists), as in (ii).

This completes the proof of the lemma.

Corollary 6.6. If $D=C_{G}(a)^{\prime}$ has a factor $D_{4}$ or $D_{5}$, then one of the following holds:

(i) $S<Q A_{1}^{k}<D$ with $k \leq 5$ (the $A_{1}^{k}$ being fundamental $A_{1}$ 's in $G$ );

(ii) $S<Q A_{1} B_{1}^{3}<A_{2} D_{4}$ or $A_{1} D_{5}$;

(iii) $S<Q A_{1}^{2} B_{1}^{2}<A_{1} D_{5}$;

(iv) $S<A_{1} B_{1}^{4}<A_{1} D_{5}$;

(v) $S<A_{1}^{3} B_{1}^{2}<A_{1} D_{5}$.

In cases (ii) $-(\mathrm{v}), S$ has distinct twists on the $B_{1}$ factors.

Proof. Observe that $D \neq A_{1} A_{1} D_{4}$, since otherwise $a \in C_{G}\left(A_{1} A_{1} D_{4}\right)=A_{1} A_{1}=$ $C_{G}\left(D_{6}\right)$, contradicting 3.7. Hence, as $D$ satisfies $3.5(5)$, we see that $D=A_{r} D_{4}$ or $A_{s} D_{5}$ with $r \leq 2, s \leq 1$. The projection $S_{0}$ of $S$ in the factor $D_{4}$ or $D_{5}$ satisfies 6.5(i) or 6.5(ii). We see from this that either the result holds, or $S_{0}$ lies in a subgroup $C_{1}^{3}$ or $Q C_{1}^{2}$ of the factor $D_{4}$ or $D_{5}$. If $S_{0}<C_{1}^{3}$, then $S_{0}<C_{1}^{3}<D_{4}$, and $N_{G}\left(D_{4}\right)$ contains a triality automorphism of this $D_{4}$. This triality sends the subgroup $C_{1}^{3}$ to a subgroup $B_{1}^{3}$ of $D_{4}$, and hence a conjugate of $S$ lies in a subgroup $A_{r} B_{1}^{3}$ or $A_{s} B_{1}^{3}$ of $D$, as in (ii) of the conclusion. A similar argument applies if $S_{0}<Q C_{1}^{2}$; here $S_{0}<Q C_{1}^{2}<D_{4}$, and application of triality gives a conjugate of $S$ lying in $A_{r}\left(Q B_{1}^{2}\right)$ or $A_{s}\left(Q B_{1}^{2}\right)$, yielding (ii) again.

In the next lemma we consider the other possibilities for $D$.

Lemma 6.7. Suppose that $D$ is a product of factors $A_{m}$. Then conclusion (i) or (v) of Corollary 6.6 holds.

Proof. Recall that $D$ is as in 3.5(5). Note also that $D \neq A_{3} A_{4}$ or $A_{1} A_{6}$ by 3.4. Assume that 6.6(i) does not hold. Then $D$ must have a factor $A_{3+\delta}(\delta \geq 0)$ with natural module $V$ such that $V \downarrow S$ has a composition factor $1^{\left(2^{i}\right)} \otimes 1^{\left(2^{j}\right)}$; and $D \neq A_{1} A_{5}$, since otherwise $a \in C_{G}\left(A_{1} A_{5}\right)=A_{2}=C_{G}\left(E_{6}\right)$, contrary to 3.6. Moreover, if $D$ has a factor $A_{4}$, then the natural module for this factor restricts to $S$ as $1^{\left(2^{i}\right)} \otimes 1^{\left(2^{j}\right)} / 0$; this splits by 1.2 , and hence $S$ projects into a subgroup $A_{3}$ of this factor. We conclude that one of the following holds:

(a) $S<D=A_{5}$ or $A_{6}$, with embedding $1^{\left(2^{i}\right)} \otimes 1^{\left(2^{j}\right)} / 1^{\left(2^{k}\right)} / 0^{m}$ or $1^{\left(2^{i}\right)} \otimes 1^{\left(2^{j}\right)} / 0^{2+m}$ $(m \leq 1)$; 
(b) $S<A_{1}^{3} A_{3}$, with projection $1^{\left(2^{i}\right)} \otimes 1^{\left(2^{j}\right)}$ in the $A_{3}$ factor;

(c) $S<A_{3} A_{3}$, with projection $1^{\left(2^{i}\right)} \otimes 1^{\left(2^{j}\right)}$ in one of the $A_{3}$ factors;

(d) $S<D=A_{1} A_{2} A_{3}$, with projection $1^{\left(2^{i}\right)} \otimes 1^{\left(2^{j}\right)}$ in the $A_{3}$ factor.

Consider first case (b), $S<A_{1}^{3} A_{3}$. Now $A_{1}^{3} A_{3}<A_{1}^{3} D_{4}$ in $G$, and $N_{G}\left(A_{1}^{3} D_{4}\right)$ contains an element $\tau$ of order 3 inducing a triality automorphism on the $D_{4}$ factor and permuting the $A_{1}$ factors cyclically. Since the projection of $S$ to the $A_{3}$ factor is $1^{\left(2^{i}\right)} \otimes 1^{\left(2^{j}\right)}$, we see that for some $k, S^{\tau^{k}}$ projects into a subgroup $B_{1}^{2}$ in the $D_{4}$ factor. Hence $S<A_{1}^{3} B_{1}^{2}<A_{1}^{3} D_{4}$. Moreover this $A_{1}^{3} B_{1}^{2}<A_{1}^{3} D_{3}<A_{1}^{3} D_{4}$, so $S<A_{1}^{3} B_{1}^{2}<A_{1}^{3} D_{3}<A_{1} D_{5}$, as in 6.6(v). Note that the twists on the $B_{1}$ factors are distinct.

Now suppose case (a) holds, and let $V$ be the natural module for $D=A_{5}$ or $A_{6}$. If $V \downarrow S$ is completely reducible then $S<A_{1} A_{3}<D$, as in case (b) handled above. So assume $V \downarrow S$ is not completely reducible. Then applying a twist to the labelling of $S$, we can take $V \downarrow S=1 \otimes 1^{\left(2^{i}\right)} / 1^{\left(2^{j}\right)} / 0^{m}$ for some $i, j$. If $j \neq 0, i$ then by $1.2, V \downarrow S=\left(1 \otimes 1^{\left(2^{i}\right)}\right) \oplus\left(1^{\left(2^{j}\right)} / 0^{m}\right)$, so $S<A_{3} A_{2}<A_{3} A_{3}$, as in case (c), which we handle below.

Thus we assume now that $j=0$ or $i$. Applying a twist, we may take $j=0$, so $V \downarrow S=1 \otimes 1^{\left(2^{i}\right)} / 1 / 0^{m}$. Recall that $q=2^{e}$ with $e \geq 4$, and the nontrivial composition factors of $L(G) \downarrow D$ are of the form $V_{D}\left(\lambda_{r}\right)$, together with adjoint modules of the factors ([LS2, §2]). If $i \leq e-3$ then we can choose a rank 1 torus $T_{1}$ in $D$ (namely $\left.T_{1}=\left\{\operatorname{diag}\left(c^{2^{i}+1}, c^{2^{i}-1}, c^{-2^{i}-1}, c^{-2^{i}+1}, c, c^{-1}, 1^{m}\right): c \in K^{*}\right\}\right)$ containing an element $t \in S$ of order $q+1$, such that $t$ and $T_{1}$ fix the same subspaces of $L(G)$, contrary to (†). If $i=e-1$, we can twist to take $V \downarrow S=1^{(2)} \otimes 1 / 1^{(2)} / 0^{m}$ and apply the same $T_{1}$ argument. And if $i=e-2$ we twist to $V \downarrow S=1^{(4)} \otimes 1 / 1^{(4)} / 0^{m}$, and the $T_{1}$ argument applies unless $q=16$. Thus to complete this case (a), assume now that

$$
q=16, \quad V \downarrow S=1 \otimes 1^{(4)} / 1 / 0^{m} .
$$

By [LS2, Table 8.1], $L(G) \downarrow A_{5}=L\left(A_{5}\right) / \lambda_{1}^{6} / \lambda_{2}^{3} / \lambda_{3}^{2} / \lambda_{4}^{3} / \lambda_{5}^{6} / 0^{11}$. Using [LS2, 2.13] we deduce from this that

$$
\begin{aligned}
L(G) \downarrow S= & \left(1 \otimes 1^{(2)}\right)^{2} /\left(1 \otimes 1^{(4)}\right)^{16} /\left(1 \otimes 1^{(8)}\right)^{2} /\left(1^{(2)} \otimes 1^{(4)}\right)^{8} / \\
& \left(1^{(2)} \otimes 1^{(8)}\right)^{1} / 1^{16} /\left(1^{(2)}\right)^{9} /\left(1^{(4)}\right)^{16} /\left(1^{(8)}\right)^{8} / 0^{34} .
\end{aligned}
$$

As $q=16$, we have $A=B_{2}(16)$ or $G_{2}(16)$. If $A=B_{2}(16)$ then $S$ contains a conjugate $b$ of $a$ (of order 17). But from $L(G) \downarrow S$ we see that $\operatorname{dim} C_{G}(b)=34$, whereas $\operatorname{dim} C_{G}(a) \geq \operatorname{dim} A_{5} T_{3}=38$, a contradiction. Now suppose $A=G_{2}(16)$. Since $L(G) \downarrow S$ has composition factor $1^{(2)} \otimes 1^{(8)}$ with multiplicity 1 , we see from Lemma 6.1 that $L(G) \downarrow A$ must have a composition factor $01 \otimes 01^{(4)}$; but the restriction of this to $S$ has composition factor $1 \otimes 1^{(8)}$ with multiplicity 4 , which contradicts $L(G) \downarrow S$ given above. This completes the argument for case (a).

Next consider case (c), $S<A_{3} A_{3}$. If $S<A_{1}^{2} A_{3}$ then we are in case (b), so assume this is not so. Then the embedding of $S$ in $A_{3} A_{3}$ is either $1^{\left(2^{i_{1}}\right)} \otimes 1^{\left(2^{i_{2}}\right)}, 1^{\left(2^{i_{3}}\right)} \otimes$ $1^{\left(2^{i_{4}}\right)}$ or $1^{\left(2^{i_{1}}\right)} \otimes 1^{\left(2^{i_{2}}\right)}, 1^{\left(2^{i_{3}}\right)} / 0^{2}$, and in the latter case the second representation is indecomposable. By [LS2, p.60], the composition factors of $L(G) \downarrow A_{3} A_{3}$ are among $L\left(A_{3} A_{3}\right), \lambda_{i} \otimes \lambda_{j}, \lambda_{i} \otimes 0$ and $0 \otimes \lambda_{j}$, and include $\lambda_{1} \otimes \lambda_{1}$.

Suppose first that the embedding is $1^{\left(2^{i_{1}}\right)} \otimes 1^{\left(2^{i_{2}}\right)}, 1^{\left(2^{i_{3}}\right)} \otimes 1^{\left(2^{i_{4}}\right)}$. If $i_{1}, \ldots, i_{4}$ are 
all distinct, then $L(G) \downarrow S$ has a composition factor $1^{\left(2^{i_{1}}\right)} \otimes 1^{\left(2^{i_{2}}\right)} \otimes 1^{\left(2^{i_{3}}\right)} \otimes 1^{\left(2^{i_{4}}\right)}$ (coming from $\lambda_{1} \otimes \lambda_{1}$ ), and either no $1^{\left(2^{i_{1}}\right)} \otimes 1^{\left(2^{i_{2}}\right)} \otimes 1^{\left(2^{i_{3}}\right)}$ or no $1^{\left(2^{i_{1}}\right)} \otimes 1^{\left(2^{i_{2}}\right)} \otimes 1^{\left(2^{i_{4}}\right)}$; this contradicts $6.2(\mathrm{i}, \mathrm{ii})$. Therefore $i_{1}, \ldots, i_{4}$ are not all distinct. If $e \geq 5$ we can twist to take $i_{1} \ldots i_{4}$ to satisfy (i), (ii) or (iii) of 6.3 . Now $S<\bar{S}<A_{3} A_{3}$, where $\bar{S} \cong S L_{2}(K)$ has embedding $1^{\left(2^{i_{1}}\right)} \otimes 1^{\left(2^{i_{2}}\right)}, 1^{\left(2^{i_{3}}\right)} \otimes 1^{\left(2^{i_{4}}\right)}$ in $A_{3} A_{3}$, and it is easily checked that $\bar{S}$ has all weights on $L(G)$ less than $q$. Then by $1.5, S$ and $\bar{S}$ fix the same subspaces of $L(G)$, contradicting $(\dagger)$. Finally suppose $e=4$. We can twist to take the embedding the embedding of $S$ in $A_{3} A_{3}$ to be

$$
\begin{gathered}
1 \otimes 1^{(2)}, 1^{(2)} \otimes 1^{(4)} \text { or } 1 \otimes 1^{(4)}, 1 \otimes 1^{(2)} \text { or } 1 \otimes 1^{(4)}, 1^{(2)} \otimes 1^{(4)} \text { or } \\
1 \otimes 1^{(2)}, 1 \otimes 1^{(2)} \text { or } 1 \otimes 1^{(4)}, 1 \otimes 1^{(4)} .
\end{gathered}
$$

Choose $\bar{S} \cong S L_{2}(K)$ with $S<\bar{S}<A_{3} A_{3}$ and $\bar{S}$ having one of these embeddings in $A_{3} A_{3}$. Then in the first, second and fourth embeddings, $\bar{S}$ has all weights on $L(G)$ less than $q=16$. In the third case, $\bar{S}$ could have a composition factor of high weight 16 coming from a composition factor $\lambda_{2} \otimes \lambda_{2}$ for $L(G) \downarrow A_{3} A_{3}$. By [LS2, p.60], the presence of a composition factor $\lambda_{2} \otimes \lambda_{2}$ implies that there are no composition factors $\lambda_{i} \otimes \lambda_{2}$ with $i \in\{1,3\}$; so we see that $\bar{S}$ has highest weight 16 on $L(G)$, and has no composition factor of high weight 1 . Hence 1.4 implies that $S$ and $\bar{S}$ fix the same subspaces of $L(G)$, contradicting $(\dagger)$. The same argument handles the final embedding $1 \otimes 1^{(4)}, 1 \otimes 1^{(4)}$.

Now suppose that the embedding of $S$ in $A_{3} A_{3}$ is $1^{\left(2^{i_{1}}\right)} \otimes 1^{\left(2^{i_{2}}\right)}, 1^{\left(2^{i_{3}}\right)} / 0^{2}$. Assume first that $e \geq 5$. Then we can take $i_{1} i_{2} i_{3}$ (in some order) to satisfy the conclusion of 6.4. If $i_{r} \leq e-3$ for all $r$, then the $T_{1}$ argument applies (i.e. there is a torus $T_{1}$ in $A_{3} A_{3}$ containing an element $t \in S$ of order $q+1$ and fixing the same subspaces of $L(G)$ as $t$, contrary to $(\dagger))$. Otherwise, by 6.4 , we have $e=5$ or 6 and $\left\{i_{1}, i_{2}, i_{3}\right\}=\{0,1,3\}$ or $\{0,2,4\}$ respectively. In the first case, either the $T_{1}$ argument applies, or the embedding of $S$ is $1^{(2)} \otimes 1^{(8)}, 1 / 0^{2}$. From the two possible restrictions $L(G) \downarrow A_{3} A_{3}$ given in [LS2, p.60], we deduce that $L(G) \downarrow S$ has 8 composition factors $1 \otimes 1^{(2)} \otimes 1^{(8)}$, but no composition factors $1 \otimes 1^{(8)}$; hence $A \neq A_{2}^{\epsilon}(q)$ by $6.2\left(\right.$ ii). Thus $A=B_{2}(q)$, whence $S$ contains a conjugate $b$ of $a$ (of order $q+1$ ). Again using [LS2, p.60], we see that $C_{G}(b)$ has dimension 34 . Now $A_{3} A_{3}$ lies in a subgroup $D_{4} D_{4}$ of $G$, which in turn lies in a subgroup $D_{8}$. Consideration of the eigenvalues of $b$ on the natural $D_{8}$-module shows that $C_{D_{8}}(b)^{\prime}$ is either $A_{1}^{5}$ or $A_{1} D_{4}$. Since $C_{G}(b)$ has dimension 34, this forces $C_{G}(b)=A_{1} D_{4} T_{3}$, contrary to the hypothesis of the lemma.

To complete case (c), assume that $e=4$ (and the embedding of $S$ in $A_{3} A_{3}$ is $\left.1^{\left(2^{i_{1}}\right)} \otimes 1^{\left(2^{i_{2}}\right)}, 1^{\left(2^{i_{3}}\right)} / 0^{2}\right)$. If we can twist to take $\left(i_{1}, i_{2}, i_{3}\right)=(0,1,2),(0,2,1),(0,1,0)$ or $(0,1,1)$, then the $T_{1}$ argument applies. The only remaining possibilities are $\left(i_{1}, i_{2}, i_{3}\right)=(1,2,0)$ or $(0,2,0)$. For $A=B_{2}(16)$ these are handled as in the previous paragraph. For $A=G_{2}(16)$ in the $(0,2,0)$ case, we find that $L(G) \downarrow S$ has composition factor $1 \otimes 1^{(2)}$ with multiplicity 2 , conflicting with 6.1 . And for $A=G_{2}(16)$ in the $(1,2,0)$ case, we work out the composition factors of $L(G) \downarrow S$ (using [LS2, p.60]), and show that these conflict with 6.2. This completes case (c).

It remains to consider case (d), $S<D=A_{1} A_{2} A_{3}$. We can take it that the projections of $S$ to each of the 3 factors are nontrivial (otherwise we are in a previous case). Say the embedding is $1^{\left(2^{i_{1}}\right)}, 1^{\left(2^{i_{2}}\right)} / 0,1^{\left(2^{i_{3}}\right)} \otimes 1^{\left(2^{i_{4}}\right)}$. Now $D$ lies in a 
maximal rank subgroup $A_{1} A_{2} A_{5}$ of $G$, and from [LS2, §2] we have

$$
\begin{aligned}
L(G) & \downarrow A_{1} A_{2} A_{5} \\
& =L\left(A_{1} A_{2} A_{5}\right) / 1 \otimes \lambda_{1} \otimes \lambda_{1} / 1 \otimes \lambda_{2} \otimes \lambda_{5} / 1 \otimes 0 \otimes \lambda_{3} / 0 \otimes \lambda_{1} \otimes \lambda_{2} / 0 \otimes \lambda_{2} \otimes \lambda_{4} .
\end{aligned}
$$

If $i_{1}, \ldots, i_{4}$ are all distinct then $L(G)$ has a composition factor $1^{\left(2^{i_{1}}\right)} \otimes \ldots \otimes 1^{\left(2^{i_{4}}\right)}$ (from $1 \otimes \lambda_{1} \otimes \lambda_{1}$ ), but no composition factor $1^{\left(2^{i_{1}}\right)} \otimes 1^{\left(2^{i_{2}}\right)} \otimes 1^{\left(2^{i_{3}}\right)}$, contrary to 6.2(i,ii). Thus $i_{1}, \ldots, i_{4}$ are not all distinct.

Assume now that $e \geq 5$. Then we can take $i_{1} \ldots i_{4}$ to satisfy the conclusion of 6.4. If $i_{r} \leq e-3$ for all $r$ then the $T_{1}$ argument applies. Otherwise, $i_{1} \ldots i_{4}$ (in some order) is one of 0013,0023, 0113 (all with $e=5$ ) or 0024 (with $e=6$ ). If $i_{2}>0$ then $S<\bar{S}<D$, where $\bar{S} \cong S L_{2}(K)$ with embedding $1^{\left(2^{i_{1}}\right)}, 1^{\left(2^{i_{2}}\right)} / 0,1^{\left(2^{i_{3}}\right)} \otimes 1^{\left(2^{i_{4}}\right)}$ (the point being that by 1.2 , for $S L_{2}(K)$, the indecomposable $1^{\left(2^{i_{2}}\right)}$ exists only if $i_{2}>0$, in which case $\left.\operatorname{Ext}_{S}\left(1^{\left(2^{i_{2}}\right)}, 0\right) \cong \operatorname{Ext}_{S L_{2}(K)}\left(1^{\left(2^{i_{2}}\right)}, 0\right)\right)$. But now $\bar{S}$ has all weights on $L(G)$ less than $q$. Hence $i_{2}=0$, and the embedding of $S$ in $D$ is one of

$$
\begin{aligned}
& 1,1 / 0,1^{(2)} \otimes 1^{(8)} \text { or } 1^{(2)}, 1 / 0,1 \otimes 1^{(8)} \text { or } 1^{(8)}, 1 / 0,1 \otimes 1^{(2)}(e=5), \\
& 1,1 / 0,1^{(4)} \otimes 1^{(8)} \text { or } 1^{(4)}, 1 / 0,1 \otimes 1^{(8)} \text { or } 1^{(8)}, 1 / 0,1 \otimes 1^{(4)}(e=5), \\
& 1^{(2)}, 1 / 0,1^{(2)} \otimes 1^{(8)}(e=5), \\
& 1,1 / 0,1^{(4)} \otimes 1^{(16)} \text { or } 1^{(4)}, 1 / 0,1 \otimes 1^{(16)} \text { or } 1^{(16)}, 1 / 0,1 \otimes 1^{(4)} \quad(e=6) .
\end{aligned}
$$

Twist the embeddings in the first and third rows by $2^{2}$, those in the second row by $2^{3}$, and those in the fourth row by $2^{2}$; so for example, the embeddings in the first row now read

$$
1^{(4)}, 1^{(4)} / 0,1^{(4)} \otimes 1 \text { or } 1^{(8)}, 1^{(4)} / 0,1^{(4)} \otimes 1 \text { or } 1,1^{(4)} / 0,1^{(4)} \otimes 1^{(8)}(e=5) .
$$

Now $S<\bar{S}<D$, with $\bar{S}$ embedded in $D$ with the new labelling, and we see that the highest weight of $\bar{S}$ on $L(G)$ is less than $q$. This completes the proof for $e \geq 5$.

To complete the proof for case (d), assume finally that $e=4$. Then we can take $i_{1} \ldots i_{4}$ (in some order) to be $0012,0112,0122$ or 0022 (in all other cases the $T_{1}$ argument applies). As above, $i_{2}=0$.

For the tuple 0012 , the embedding of $S$ is $1,1 / 0,1^{(2)} \otimes 1^{(4)}$ or $1^{(2)}, 1 / 0,1 \otimes 1^{(4)}$ or $1^{(4)}, 1 / 0,1 \otimes 1^{(2)}$. In the last case, the $T_{1}$ argument applies. In the second case, $L(G) \downarrow S$ has composition factor $1 \otimes 1^{(2)} \otimes 1^{(4)}$ with multiplicity 3 , so $A \neq G_{2}(16)$ by 6.2 (iii); also if $b \in S$ of order 17 , we calculate from $L(G) \downarrow D$ above that $C_{L(G)}(b)$ has dimension 24, so $A \neq B_{2}(16)$ (as otherwise $b$ is conjugate to $a$, whereas $C_{G}(a)=A_{1} A_{2} A_{3} T_{2}$, of dimension 28). In the first case, we twist to take the embedding to be $1^{(2)}, 1^{(2)} / 0,1^{(4)} \otimes 1^{(8)}$. As explained before, we have $S<\bar{S}<D$, where $\bar{S} \cong S L_{2}(K)$ has this embedding in $D$. The composition factors of $\bar{S}$ on $L(G)$ of high weight 16 or more are $1^{(16)}$ and $1^{(2)} \otimes 1^{(16)}$, both of which are irreducible for $S$; moreover, $\bar{S}$ has no composition factors 1 or $1^{(2)} \otimes 1$. Hence $S$ and $\bar{S}$ fix the same subspaces of $L(G)$, by 1.4 , contradicting $(\dagger)$.

The other tuples 0112,0122 and 0022 are handled in a similar fashion. This completes the proof of the lemma.

In view of the lemma just proved, it remains to deal with $S$ as in cases (i)-(v) of Corollary 6.6. To handle cases (ii)-(v), we require information about the restriction of $L(G)$ to the subgroups $A_{1} B_{1}^{4}$ and $A_{1}^{2} B_{1}^{3}$, which is provided by the next lemma. In the conclusion, by a "1-fold tensor" we mean a module for the product $A_{1} B_{1}^{4}$ or $A_{1}^{2} B_{1}^{3}$ in which only one of the simple factors acts nontrivially. 
Lemma 6.8. (i) For $A_{1} B_{1}^{4}<A_{1} D_{5}<G$, the nontrivial composition factors of $L(G) \downarrow A_{1} B_{1}^{4}$ are as follows:

1-fold tensors 1 or $1^{(2)}$,

$0 \otimes 1^{(2)} \otimes 1^{(2)} \otimes 0 \otimes 0, \ldots, 0 \otimes 0 \otimes 0 \otimes 1^{(2)} \otimes 1^{(2)} \quad$ (one for each pair

of $B_{1}$ factors, each with mult. 1),

$1 \otimes 1^{(2)} \otimes 0 \otimes 0 \otimes 0, \ldots, 1 \otimes 0 \otimes 0 \otimes 0 \otimes 1^{(2)} \quad$ (one for each $B_{1}$ factor, each with mult. 2),

$1 \otimes 1 \otimes 1 \otimes 1 \otimes 1$ (mult. 2), $0 \otimes 1 \otimes 1 \otimes 1 \otimes 1$ (mult. 4).

(ii) For $A_{1}^{3} B_{1}^{2}=A_{1}\left(A_{1}^{2} B_{1}^{2}\right)<A_{1}\left(D_{2} D_{3}\right)<A_{1} D_{5}<G$, the nontrivial composition factors of $L(G) \downarrow A_{1}^{3} B_{1}^{2}$ are:

$$
\begin{aligned}
& \text { 1-fold tensors } 1 \text { or } 1^{(2)} \text { (only } 1^{(2)} \text { for } B_{1} \text { factors), } \\
& 0 \otimes 1 \otimes 1 \otimes 0 \otimes 0,0 \otimes 1 \otimes 1 \otimes 1^{(2)} \otimes 0,0 \otimes 1 \otimes 1 \otimes 0 \otimes 1^{(2)}, \\
& 0 \otimes 0 \otimes 0 \otimes 1^{(2)} \otimes 1^{(2)}, 1 \otimes 1 \otimes 1 \otimes 0 \otimes 0,1 \otimes 0 \otimes 0 \otimes 1^{(2)} \otimes 0, \\
& 1 \otimes 0 \otimes 0 \otimes 0 \otimes 1^{(2)}, 0 \otimes 1 \otimes 0 \otimes 1 \otimes 1,0 \otimes 0 \otimes 1 \otimes 1 \otimes 1, \\
& 1 \otimes 1 \otimes 0 \otimes 1 \otimes 1,1 \otimes 0 \otimes 1 \otimes 1 \otimes 1 \text { (both with mult. } 2 \text { ). }
\end{aligned}
$$

Proof. By $[\mathrm{LS} 2, \S 2], L(G) \downarrow A_{3} D_{5}=L\left(A_{3}\right) / L\left(D_{5}\right) / \lambda_{2} \otimes \lambda_{1} / \lambda_{1} \otimes \lambda_{4} / \lambda_{3} \otimes \lambda_{5}$, whence $L(G) \downarrow A_{1} D_{5}$

$$
=\left(L\left(A_{3}\right) \downarrow A_{1}\right) / L\left(D_{5}\right) /\left(1 \otimes \lambda_{1}\right)^{2} /\left(0 \otimes \lambda_{1}\right)^{2} / 1 \otimes \lambda_{4} /\left(0 \otimes \lambda_{4}\right)^{2} / 1 \otimes \lambda_{5} /\left(0 \otimes \lambda_{5}\right)^{2} .
$$

Let $V=V_{D_{5}}\left(\lambda_{1}\right)$, the usual 10-dimensional module. Then from the definition of $B_{1}^{4}<D_{5}$, we have $V \downarrow B_{1}^{4}=1^{(2)} / 1^{(2)} / 1^{(2)} / 1^{(2)} / 0^{2}$ (where $1^{(2)}$ stands for a 1 -fold tensor, one for each factor $B_{1}$ ), and $V \downarrow A_{1}^{2} B_{1}^{2}=1 \otimes 1 / 1^{(2)} / 1^{(2)} / 0^{2}$ (where $1 \otimes 1$ has the $A_{1}^{2}$ acting, and the $1^{(2)}$ have a single factor $B_{1}$ acting). It follows, using [LS2, 2.6 and 2.7], that for $m=4,5$, we have

$$
V_{D_{5}}\left(\lambda_{m}\right) \downarrow B_{1}^{4}=1 \otimes 1 \otimes 1 \otimes 1, \quad V_{D_{5}}\left(\lambda_{m}\right) \downarrow A_{1}^{2} B_{1}^{2}=1 \otimes 0 \otimes 1 \otimes 1 / 0 \otimes 1 \otimes 1 \otimes 1 .
$$

Using this and the above description of $L(G) \downarrow A_{1} D_{5}$, we obtain the conclusion.

Lemma 6.9. Case (iv) of 6.6 does not hold.

Proof. Suppose 6.6(iv) holds, so $S<A_{1} B_{1}^{4}$ with distinct twists of the $B_{1}$ factors. If $S$ projects nontrivially to each $B_{1}$ factor, say with projections $1^{\left(2^{i_{1}}\right)}, \ldots, 1^{\left(2^{i_{4}}\right)}$, then we see from 6.8(i) that $L(G) \downarrow S$ has a composition factor $1^{\left(2^{i_{1}}\right)} \otimes \ldots \otimes 1^{\left(2^{i_{4}}\right)}$, but no composition factor $\bigotimes_{r \in A} 1^{\left(2^{i_{r}}\right)}$ for some 3 -set $A \subseteq\{1,2,3,4\}$. This contradicts $6.2(\mathrm{i}, \mathrm{ii})$.

Hence $S$ projects trivially to some $B_{1}$ factor. Let $1^{\left(2^{i_{1}}\right)}, \ldots, 1^{\left(2^{i} k\right)}$ be the nontrivial projections of $S$ into the factors of $A_{1} B_{1}^{4}$; thus $k \leq 4$. Then $S<\bar{S}<A_{1} B_{1}^{4}$, where $\bar{S} \cong S L_{2}(K)$ also has these projections.

If $e \geq 5$ then we can twist to take $i_{r} \leq e-2$ for all $r$. Then from 6.8(i) we see that the weights of $\bar{S}$ on $L(G)$ are all less than $q$. Then by $1.5, S$ and $\bar{S}$ fix the same subspaces of $L(G)$, contradicting $(\dagger)$.

Now suppose $e=4$. If $S$ projects trivially into two or more of the $B_{1}$ factors, then we can twist to take $i_{r} \leq 2$ for all $r$, giving a contradiction as above. Therefore $S$ projects trivially into just one $B_{1}$ factor. We can take the projections into the other $B_{1}$ factors to be $1,1^{(2)}, 1^{(4)}$; then the weights of $\bar{S}$ on $L(G)$ are all less than $q$, unless the projection into the $A_{1}$ factor is $1^{(8)}$. But then by $6.8(\mathrm{i}), L(G) \downarrow S$ has a composition factor $1 \otimes 1^{(2)} \otimes 1^{(4)} \otimes 1^{(8)}$, contrary to $6.2\left(\right.$ i) (recall that $A=B_{2}(16)$ or $G_{2}(16)$ when $\left.q=16\right)$. 
Lemma 6.10. Case (v) of 6.6 does not hold.

Proof. Assume 6.6(v) holds, so $S<A_{1}^{3} B_{1}^{2}$ with distinct twists on the $B_{1}$ factors. Observe that $S<\bar{S}<A_{1}^{3} B_{1}^{2}$, where $\bar{S} \cong S L_{2}(K)$.

Suppose first that $e \geq 5$. If $S$ has distinct nontrivial twists on all five factors of $A_{1}^{3} B_{1}^{2}$, we obtain a contradiction using 6.8(ii) together with 6.2(i,ii). Otherwise, we can twist to take all nontrivial projections of $\bar{S}$ to be of the form $1^{\left(2^{i}\right)}$ with $i \leq e-2$, and then the highest weight of $\bar{S}$ on $L(G)$ is less than $q$, giving the usual contradiction.

Now suppose $e=4$. Assume first that $S$ projects nontrivially into all five factors of $A_{1}^{3} B_{1}^{2}$, with projections $1^{\left(2^{i_{1}}\right)}, \ldots, 1^{\left(2^{i_{5}}\right)}$. If all $i_{r} \leq 2$ then, twisting if necessary, $\bar{S}$ has all weights less than $q$, so some $i_{r}=3$. Then we can twist to take $i_{1} \ldots i_{5}$ (in some order) to be 00123. Now $L(G) \downarrow S$ has no 4-fold tensor composition factor, by 6.2(i); it follows from 6.8(ii) that (with the factors ordered as in 6.8(ii)), the projections of $S$ in $A_{1} A_{1} A_{1} B_{1} B_{1}$ are $1,1^{(2)}, 1^{(4)}, 1,1^{(8)}$ or $1,1^{(2)}, 1^{(8)}, 1,1^{(4)}$ or $1,1^{(4)}, 1^{(8)}, 1,1^{(2)}$. In the first case, by $6.8(\mathrm{ii}), L(G) \downarrow S$ has a composition factor $1 \otimes 1^{(4)} \otimes 1^{(8)}$, but no composition factor $1 \otimes 1^{(8)}$, contrary to $6.2(\mathrm{iii})$. We obtain similar contradictions in the other cases.

Therefore $S$ projects trivially to at least one of the factors of $A_{1}^{3} B_{1}^{2}$. The only case where $\bar{S}$ cannot be chosen to have all weights less than $q$ is that in which $S$ has four nontrivial projections $1,1^{(2)}, 1^{(4)}, 1^{(8)}$. By $6.2(\mathrm{i}), L(G) \downarrow S$ has no 4 -fold tensor composition factor. Hence by 6.8 (ii), we see that the projections of $S$ in $A_{1} A_{1} A_{1} B_{1} B_{1}$ can be taken to be one of the following (up to reordering the $3 A_{1}$ factors and the $2 B_{1}$ factors):

(a) $1^{\left(2^{i_{1}}\right)}, 1^{\left(2^{i_{2}}\right)}, 1^{\left(2^{i_{3}}\right)}, 1,0^{2}$ (where $\left\{i_{1}, i_{2}, i_{3}\right\}=\{1,2,3\}$ ),

(b) $0^{2}, 1^{(4)}, 1^{(8)}, 1,1^{(2)}$,

(c) $0^{2}, 1^{(2)}, 1^{(8)}, 1,1^{(4)}$.

In cases (a) and (b), the highest weight of $\bar{S}$ on $L(G)$ is 16 , and there are no composition factors of high weight 1 , so 1.4 implies that $S$ and $\bar{S}$ fix the same subspaces of $L(G)$, a contradiction. And in case (c), the composition factors of $\bar{S}$ having high weight 16 or more are $1^{(16)}$ and $1^{(2)} \otimes 1^{(16)}$; using $6.8($ ii) we check that $\bar{S}$ has no composition factors 1 or $1^{(2)} \otimes 1$, so again 1.4 gives a contradiction.

The next lemma is useful in dealing with case (ii) of 6.6, in which $S<Q A_{1} B_{1}^{3}$, lying in either $A_{2} D_{4}$ or $A_{1} D_{5}$. In the conclusion there is a certain subgroup $B_{1} B_{1}^{\prime} B_{1}^{\prime \prime}$ of $D_{5}$; this is a commuting product of three subgroups $S O_{3}$ of $S O_{10}$, where the radicals of the associated 3-spaces may be different.

Lemma 6.11. Let $S_{0}=S L_{2}\left(2^{e}\right)$ (e $\left.\geq 4\right)$. Suppose that $S_{0}<Q B_{1}^{3}<D_{5}$, and $S_{0}$ has three distinct nontrivial twists on the $B_{1}$ factors. Then there is a subgroup $B_{1} B_{1}^{\prime} B_{1}^{\prime \prime} \cong B_{1}^{3}$ of $D_{5}$ such that $Q B_{1}^{3}=Q B_{1} B_{1}^{\prime} B_{1}^{\prime \prime}$ and $S_{0}<B_{1} B_{1}^{\prime} B_{1}^{\prime \prime}$.

Proof. Let $V_{10}$ be the usual 10-dimensional $D_{5}$-module. Now $Q B_{1}^{3}<Q D_{4}<D_{5}$, so $S_{0}$ fixes both a singular vector $v$ and a nonsingular vector $w$ in $V_{10}$. Write $V_{8}$ for the $C_{4}$-space $w^{\perp} /\langle w\rangle$. By hypothesis, $V_{8} \downarrow S_{0}$ has three nontrivial composition factors $1^{\left(2^{i}\right)}, 1^{\left(2^{j}\right)}, 1^{\left(2^{k}\right)}$, where $i, j, k$ are distinct. Pulling back to $V_{10}$, we see that $S_{0}$ fixes just three indecomposable 3 -spaces in $V_{10}$ of the form $0 / 1^{\left(2^{i}\right)}, 0 / 1^{\left(2^{j}\right)}$ and $0 / 1^{\left(2^{k}\right)}$; call these $W_{i}, W_{j}, W_{k}$ respectively, and let $w_{i}, w_{j}, w_{k}$ be nonzero vectors fixed by $S_{0}$ in $W_{i}, W_{j}, W_{k}$. 
Now $\left(D_{5}\right)_{W_{i}}$ is a subgroup $B_{1} B_{3}$ of $B_{4}=\left(D_{5}\right)_{w_{i}}$; similarly $\left(D_{5}\right)_{W_{j}}=B_{1}^{\prime} B_{3}^{\prime}$ and $\left(D_{5}\right)_{W_{k}}=B_{1}^{\prime \prime} B_{3}^{\prime \prime}$. The group $B_{1}$ acts trivially on the image of $W_{j} \bmod \left\langle w_{i}\right\rangle$, and also on $\left\langle w_{i}\right\rangle$, hence acts trivially on $W_{j}$, and similarly on $W_{k}$. Hence $B_{1}$ lies in the kernel of the action of $\left(D_{5}\right)_{W_{j}}$ on $W_{j}$, which is $B_{3}^{\prime}$, and consequently $\left[B_{1}, B_{1}^{\prime}\right]=1$. Similarly $\left[B_{1}, B_{1}^{\prime \prime}\right]=\left[B_{1}^{\prime}, B_{1}^{\prime \prime}\right]=1$. Finally, $\left(D_{5}\right)_{W_{i}, W_{j}, W_{k}}=B_{1} B_{1}^{\prime} B_{1}^{\prime \prime}$, so this group contains $S_{0}$.

Lemma 6.12. Case (ii) of 6.6 does not hold.

Proof. Suppose 6.6(ii) holds, so $S<Q A_{1} B_{1}^{3}$, lying in either $A_{1} D_{5}$ or $A_{2} D_{4}$, and $S$ has distinct twists on the $B_{1}$ factors. Moreover, by $6.7, D=C_{G}(a)^{\prime}$ is not a product of factors $A_{m}$, and hence $D$ has a factor $D_{4}$ or $D_{5}$.

Suppose first that the projections of $S$ to the factors $A_{1} B_{1}^{3}$ are all nontrivial; say they are $1^{2^{\left(i_{1}\right)}}, \ldots, 1^{2^{\left(i_{4}\right)}}$. If these are all distinct, then by $6.8(\mathrm{i}), L(G) \downarrow S$ has a composition factor $1^{2^{\left(i_{1}\right)}} \otimes \ldots \otimes 1^{2^{\left(i_{4}\right)}}$, but no $1^{2^{\left(_{1}\right)}} \otimes 1^{\left(2^{i_{3}}\right)} \otimes 1^{2^{\left(i_{4}\right)}}$, contrary to $6.2(\mathrm{i}, \mathrm{ii})$. Hence $i_{1}, \ldots, i_{4}$ are not all distinct; since the $B_{1}$ twists are distinct, we may take $i_{1}=i_{2}$ (where $i_{1}$ is the twist on the $A_{1}$ factor), and $i_{2}, i_{3}, i_{4}$ are distinct.

If $Q A_{1} B_{1}^{3}<A_{1} D_{5}$, then by the previous lemma we have $S<A_{1} B_{1} B_{1}^{\prime} B_{1}^{\prime \prime}$, and the conclusion follows from 6.9. Therefore $Q A_{1} B_{1}^{3}<A_{2} D_{4}$, with $Q A_{1}<A_{2}$ and $B_{1}^{3}<D_{4}$. Observe that if $i_{1}>0$, then $S<\bar{S}<A_{2} D_{4}$ with $\bar{S} \cong S L_{2}(K)$, by 1.2 .

When $e \geq 5$, we can take $i_{1} \ldots i_{4}$ to be as in (i), (ii), or (iii) of 6.4. In case (i), the usual $T_{1}$ argument applies: there is a rank 1 torus $T_{1}$ in $A_{1} B_{1}^{3}$ fixing the same subspaces of $L(G)$ as some element of order $q+1$ in $S$. In cases (ii) and (iii) of 6.4, we have $\left(i_{1}, \ldots, i_{4}\right)=(0,0,1,3),(0,0,2,3),(1,1,0,3)(e=5)$ or $(0,0,2,4)(e=6)$. For the $(1,1,0,3)$ case, we have $S<\bar{S}<A_{2} D_{4}$ as observed above, and $\bar{S}$ has all weights on $L(G)$ less than $q$. In the $(0,0,1,3),(0,0,2,3),(0,0,2,4)$ cases, $L(G) \downarrow S$ has composition factors $1 \otimes 1^{(2)} \otimes 1^{(8)}, 1 \otimes 1^{(4)} \otimes 1^{(8)}, 1 \otimes 1^{(4)} \otimes 1^{(16)}$ (respectively), but no $1 \otimes 1^{(8)}, 1 \otimes 1^{(4)}, 1 \otimes 1^{(4)}$ (respectively), so $A \neq A_{2}^{\epsilon}(q)$ by 6.2 (ii). And for $A=B_{2}(q)$, picking a conjugate $b$ of $a$ lying in $S$, we find from $L(G) \downarrow A_{2} D_{4}$ that $C_{L(G)}(b)$ has dimension 22 , contradicting the fact that $C_{G}(a)^{\prime}$ has a factor $D_{4}$ or $D_{5}$.

To complete the case of four nontrivial projections, consider $e=4$. Here we can take $\left(i_{1}, \ldots, i_{4}\right)=(0,0,1,2),(1,0,1,2)$ or $(2,0,1,2)$. Then $L(G) \downarrow S$ has composition factors $1 \otimes 1^{(2)} \otimes 1^{(4)}, 1 \otimes 1^{(4)}$, the latter with multiplicity less than 8 , so $A \neq G_{2}(16)$ by $6.2(\mathrm{iv})$. And for $A=B_{2}(16)$ we find that $\operatorname{dim} C_{L(G)}(a)=30$ or 26 , which is impossible.

Now suppose $S$ has just three nontrivial projections to the factors of $A_{1} B_{1}^{3}$, say $1^{2^{\left(i_{1}\right)}}, 1^{\left(2^{i_{2}}\right)}, 1^{2^{\left(i_{3}\right)}}$. If the projection to the $A_{1}$ factor is trivial, then using the previous lemma we see that either $S<B_{1}^{3}<D_{4}$ or $S<B_{1} B_{1}^{\prime} B_{1}^{\prime \prime}<D_{5}$, and the conclusion follows from 6.9. Hence the projection to the $A_{1}$ factor is nontrivial; say it is $1^{2^{\left(i_{1}\right)}}$.

When $i_{r} \leq e-3$ for all $r$, the usual $T_{1}$ argument gives a contradiction. Hence by 6.4 , we can take $i_{1} i_{2} i_{3}$ (in some order) to be 012,013 or 024 with $e=4,5$ or 6 , respectively.

For the $e=6$ case, we can twist to take $\left(i_{1}, i_{2}, i_{3}\right)=(4,2,0)$, and now the usual $T_{1}$ argument works.

Now consider the $e=5$ case. If $i_{1}=3$, the $T_{1}$ argument works; and if $i_{1}=1$, we twist to take $\left(i_{1}, i_{2}, i_{3}\right)=(3,2,0)$, and now the $T_{1}$ argument again works. In the remaining case, $\left(i_{1}, i_{2}, i_{3}\right)=(0,1,3)$. Here $L(G) \downarrow S$ has a composition factor 
$1 \otimes 1^{(2)} \otimes 1^{(8)}$, but no $1 \otimes 1^{(2)}$, so $A \neq A_{2}^{\epsilon}(q)$ by $6.2\left(\right.$ ii). Thus $A=B_{2}(32)$, and $S$ contains a conjugate $b$ of $a$ of order 33. Now $S<Q A_{1} B_{1}^{2}<A_{2} D_{4}$ or $A_{1} D_{5}$, from which we calculate that $C_{L(G)}(b)$ has dimension 34 , whence $C_{G}(a)^{0}=A_{1} D_{4} T_{3}$. Thus $S<Q A_{1} B_{1}^{2}<A_{1} D_{4}$, where $Q B_{1}^{2}<D_{4}$. The proof of 6.11 shows that in fact $S<A_{1} B_{1} B_{1}^{\prime}<A_{1} D_{4}$, where $Q B_{1}^{2}=Q B_{1} B_{1}^{\prime}$. Hence $S<\bar{S}<A_{1} D_{4}$, and $\bar{S}$ has all weights on $L(G)$ less than $q$.

To conclude the case of three nontrivial projections, consider the $e=4$ case, in which $\left\{i_{1}, i_{2}, i_{3}\right\}=\{0,1,2\}$. We find that $L(G) \downarrow S$ has a composition factor $1 \otimes 1^{(2)} \otimes 1^{(4)}$, but either no $1 \otimes 1^{(4)}$ or no $1^{(2)} \otimes 1^{(4)}$, so $A \neq G_{2}(16)$. Thus $A=B_{2}(16)$, and working with a conjugate $b$ of $a$ lying in $S$ we calculate that $C_{L(G)}(b)$ has dimension 34,38 or 38 , according as $i_{1}=0,1$ or 2 ; consequently $C_{G}(a)^{\prime}$ is $A_{1} D_{4}, A_{2} D_{4}$ or $A_{2} D_{4}$ in the respective cases. Now it follows as before that $S<\bar{S}<C_{G}(a)^{\prime}$, where $\bar{S}$ has all weights on $L(G)$ less than $q$.

Finally, when $S$ has fewer than three nontrivial projections, the usual $T_{1}$ argument works in all cases, after twisting suitably. This completes the proof.

We next handle case (i) of 6.6 , in which $S<Q A_{1}^{k}<D$ with $k \leq 5$ (and $A_{1}^{k}$ is a commuting product of fundamental $A_{1}$ 's).

Lemma 6.13. Case (i) of 6.6 does not hold if $q \geq 32$.

Proof. We begin by discussing the composition factors of $L(G) \downarrow A_{1}^{5}$. Now $A_{1}^{5}$ lies in a maximal commuting product $A_{1}^{8}$ of fundamental $A_{1}$ 's in $G$, and $N_{G}\left(A_{1}^{8}\right) / A_{1}^{8} \cong$ $A G L_{3}(2)$, acting 3 -transitively on the set of 8 factors (see [As, Theorem 2]). Further, $A_{1}^{8}$ lies in the subgroup $D_{4} D_{4}$ of $G$, with each $D_{4}$ containing $A_{1}^{4}$. As $A G L_{3}(2)$ has only one orbit on 5 -sets, we may take our $A_{1}^{5}$ to lie in $D_{4} D_{4}$, with 4 of the $A_{1}$ factors lying in a $D_{4}$ factor; thus $A_{1}^{5}<A_{1} D_{4}<D_{4} D_{4}<G$. Now by [LS2, 2.1], $L(G) \downarrow D_{4} D_{4}=L\left(D_{4} D_{4}\right) / \lambda_{1} \otimes \lambda_{1} / \lambda_{3} \otimes \lambda_{3} / \lambda_{4} \otimes \lambda_{4}$, whence

$$
\begin{aligned}
L(G) \downarrow A_{1} D_{4}= & \left(L\left(D_{4}\right) \downarrow A_{1}\right) / L\left(D_{4}\right) /\left(1 \otimes \lambda_{1}\right)^{2} /\left(0 \otimes \lambda_{1}\right)^{4} /\left(1 \otimes \lambda_{3}\right)^{2} / \\
& \left(0 \otimes \lambda_{3}\right)^{4} /\left(1 \otimes \lambda_{4}\right)^{2} /\left(0 \otimes \lambda_{4}\right)^{4} .
\end{aligned}
$$

Moreover,

$$
\begin{aligned}
& L\left(D_{4}\right) \downarrow A_{1}^{4}=1 \otimes 1 \otimes 1 \otimes 1 / 1^{(2)} / 1^{(2)} / 1^{(2)} / 1^{(2)} / 0^{4}, \\
& V_{D_{4}}\left(\lambda_{1}\right) \downarrow A_{1}^{4}=1 \otimes 1 \otimes 0 \otimes 0 / 0 \otimes 0 \otimes 1 \otimes 1, \\
& V_{D_{4}}\left(\lambda_{3}\right) \downarrow A_{1}^{4}=1 \otimes 0 \otimes 1 \otimes 0 / 0 \otimes 1 \otimes 0 \otimes 1, \\
& V_{D_{4}}\left(\lambda_{4}\right) \downarrow A_{1}^{4}=1 \otimes 0 \otimes 0 \otimes 1 / 0 \otimes 1 \otimes 1 \otimes 0 .
\end{aligned}
$$

From this we see that $L(G) \downarrow A_{1}^{5}$ has no 5 -fold tensor composition factors, and has a unique 4 -fold tensor composition factor (coming from $L\left(D_{4}\right) \downarrow A_{1}^{4}$ ).

Suppose now that $q=2^{e} \geq 32$, and $S<Q A_{1}^{k}$ with nontrivial projections $1^{2^{\left(i_{1}\right)}}, \ldots, 1^{2^{\left(i_{k}\right)}}(k \leq 5)$ in to the $A_{1}$ factors. If $i_{1} \ldots i_{k}$ is as in (i) or (ii) of 6.3 , then the usual $T_{1}$ argument works: an element $b$ of order $q+1$ in $S$ lies in a rank 1 torus

$$
T_{1}=\left\{\left(c^{2^{i_{1}}}, \ldots, c^{2^{i_{k}}}\right): c \in K^{*}\right\}<A_{1}^{k}
$$

(where each entry $c^{2^{i_{r}}}$ stands for the matrix $\operatorname{diag}\left(c^{2^{i_{r}}}, c^{-2^{i_{r}}}\right)$ in the corresponding factor $A_{1}$ ); the weights of $T_{1}$ on $L(G)$ are all at most $2^{e-1}$, so $b$ and $T_{1}$ fix the same subspaces of $L(G)$, contrary to $(\dagger)$.

Therefore by 6.3 , we have $e=k=5$, and we may take $i_{1} \ldots i_{5}$ to be one of the following (in some order):

01234, 01223, 00223, 01133, 01233. 
Take $1^{\left(2^{i_{1}}\right)}$ to be the projection of $S$ into the factor $A_{1}$ of $A_{1}^{5}$ not lying in the $D_{4}$ chosen above (where $A_{1}^{5}<A_{1} D_{4}$ ).

If $i_{1} \ldots i_{5}=01234$, then from the above description, $L(G) \downarrow S$ has a unique composition factor $1^{2^{\left(i_{2}\right)}} \otimes \ldots \otimes 1^{2^{\left(i_{5}\right)}}$, contrary to $6.2(\mathrm{i})$.

Now suppose $i_{1} \ldots i_{5}=01223$. For this case, the above $T_{1}$ argument works unless $i_{1}=1$, in which case we calculate that

$$
\begin{aligned}
L(G) \downarrow S= & \left(1 \otimes 1^{(2)} \otimes 1^{(4)}\right)^{4} /\left(1 \otimes 1^{(2)} \otimes 1^{(8)}\right)^{2} /\left(1^{(2)} \otimes 1^{(4)} \otimes 1^{(8)}\right)^{4} / \\
& \left(1^{(2)} \otimes 1^{(8)}\right)^{2} /\left(1 \otimes 1^{(2)}\right)^{0} / \ldots
\end{aligned}
$$

If $A=A_{2}^{\epsilon}(q)$, the presence of ten 3-fold tensor composition factors in $L(G) \downarrow S$ implies the same for $L(G) \downarrow A$, whence $\operatorname{dim} L(G) \geq 10.27$, a contradiction. And if $A=B_{2}(q)$, the composition factors $\left(1 \otimes 1^{(2)} \otimes 1^{(8)}\right)^{2} /\left(1^{(2)} \otimes 1^{(8)}\right)^{2} /\left(1 \otimes 1^{(2)}\right)^{0}$ conflict with $6.2(\mathrm{v})$.

Next consider $i_{1} \ldots i_{5}=00223$. The usual $T_{1}$ argument works unless $i_{1}=0$, so suppose this is the case. Then

$$
L(G) \downarrow S=\left(1 \otimes 1^{(4)} \otimes 1^{(8)}\right)^{4} /\left(1 \otimes 1^{(16)}\right)^{1} /\left(1^{(16)}\right)^{1} / \ldots
$$

and the $\left(1 \otimes 1^{(4)} \otimes 1^{(8)}\right)^{4}$ are the only 3-fold tensor composition factors appearing. If $A=A_{2}^{\epsilon}(q)$, then the single factor $1 \otimes 1^{(16)}$ must occur in the restriction of a selfdual composition factor of $L(G) \downarrow A$, which must be $11^{(8)} \otimes 11^{(16)}$ (see 6.1); but the restriction of this module to $S$ has composition factor $1^{(16)}$ with multiplicity 6 , a contradiction. And if $A=B_{2}(q)$, then the single factor $1 \otimes 1^{(16)}$ must occur in the restriction of a factor $01 \otimes 01^{(16)}$ in $L(G) \downarrow A$ (see 6.1), but the restriction of this to $S$ has $1^{(16)}$ with multiplicity 2 .

Now suppose $i_{1} \ldots i_{5}=01133$. If $i_{1}=1$ or 3 , we find that

$$
L(G) \downarrow S=\left(1 \otimes 1^{(2)} \otimes 1^{(16)}\right)^{1} /\left(1 \otimes 1^{(16)}\right)^{0} / \ldots \text { or }\left(1 \otimes 1^{(4)} \otimes 1^{(8)}\right)^{1} /\left(1 \otimes 1^{(4)}\right)^{0} / \ldots
$$

respectively, contrary to 6.2 (iii). Thus $i_{1}=0$. Here

$$
L(G) \downarrow S=\left(1 \otimes 1^{(2)} \otimes 1^{(8)}\right)^{8} /\left(1 \otimes 1^{(2)}\right)^{0} / \ldots,
$$

so $A \neq A_{2}^{\epsilon}(q)$ by $6.2\left(\right.$ ii). Hence $A=B_{2}(q)$, and $S$ contains a conjugate $b$ of $a$. We calculate that $C_{G}(b)$ has dimension 34. As $S<Q A_{1}^{5}<C_{G}(a)^{\prime}$, it follows that $C_{G}(a)^{\prime}=A_{1} D_{4}$. Since a subgroup $A_{1}^{4}$ of $D_{4}$ cannot normalize a nontrivial unipotent group therein, it follows that $Q=1$, and hence $S<\bar{S}<A_{1} D_{4}$, where $\bar{S} \cong S L_{2}(K)$ has all weights on $L(G)$ less than $q$.

To conclude, let $i_{1} \ldots i_{5}=01233$. If $i_{1}=3$ then $L(G) \downarrow S$ has a unique 4 -fold tensor composition factor, contrary to $6.2(\mathrm{i})$. Otherwise, according as $i_{1}=0,1$ or 2 , we find that

$$
\begin{aligned}
L(G) \downarrow S= & \left(1^{(2)} \otimes 1^{(4)} \otimes 1^{(16)}\right)^{1} /\left(1^{(4)} \otimes 1^{(16)}\right)^{0} / \ldots, \\
& \text { or }\left(1 \otimes 1^{(4)} \otimes 1^{(16)}\right)^{1} /\left(1^{(4)} \otimes 1^{(16)}\right)^{0} / \ldots \\
& \text { or }\left(1 \otimes 1^{(2)} \otimes 1^{(16)}\right)^{1} /\left(1^{(2)} \otimes 1^{(16)}\right)^{0} / \ldots,
\end{aligned}
$$

respectively. All of these possibilities conflict with 6.2(iii). This completes the proof.

Lemma 6.14. Case (i) of 6.6 does not hold if $q=16$. 
Proof. As in the previous proof, let $S<Q A_{1}^{k}(k \leq 5)$, with nontrivial projections $1^{2^{\left(i_{1}\right)}}, \ldots, 1^{2^{\left(i_{k}\right)}}$. If $k \leq 3$, we can take $i_{1} \ldots i_{k}$ to be one of $1,11,14,111,112,114$, 122,124 , and the usual $T_{1}$ argument works in all cases.

Now suppose $k=4$. Then either the $T_{1}$ argument works, or we can twist to take $i_{1} \ldots i_{4}$ to be one of the following:

$$
\text { 0123, 0122, 0112, } 0022 .
$$

Consider the first case. If $S<Q A_{1}^{4}$ with the $A_{1}^{4}$ lying in a subgroup $D_{4}$ of $G$, then $L(G) \downarrow S$ has a 4 -fold tensor factor, contrary to $6.2(\mathrm{i})$. Otherwise, we find that

$$
\begin{aligned}
L(G) \downarrow S= & \left(1 \otimes 1^{(2)} \otimes 1^{(4)}\right)^{2} /\left(1 \otimes 1^{(2)} \otimes 1^{(8)}\right)^{2} /\left(1 \otimes 1^{(4)} \otimes 1^{(8)}\right)^{2} / \\
& \left(1^{(2)} \otimes 1^{(4)} \otimes 1^{(8)}\right)^{2} / \ldots
\end{aligned}
$$

Hence we see using 6.1 that $\operatorname{dim} L(G) \geq 4.4^{3}$ if $A=B_{2}(16)$, and $\operatorname{dim} L(G) \geq 2.6^{3}$ if $A=G_{2}(16)$, both of which are false.

Now suppose $i_{1} \ldots i_{4}=0122$. If $S<Q A_{1}^{4}$ with the $A_{1}^{4}<D_{4}$, then $L(G) \downarrow S=$ $\left(1 \otimes 1^{(2)} \otimes 1^{(8)}\right)^{1} /\left(1 \otimes 1^{(8)}\right)^{0} / \ldots$, contrary to 6.2 (iii). So assume the $A_{1}^{4} \nless D_{4}$. Then $L(G) \downarrow S=\left(1 \otimes 1^{(2)} \otimes 1^{(4)}\right)^{4} / \ldots$, ruling out $A=G_{2}(16)$ by 6.2 (iv). Thus $A=B_{2}(16)$, and $S$ contains a conjugate $b$ of $a$. We can choose a subgroup $D_{4} D_{4}$ containing $b$, and lying in a subgroup $D_{8}$ of $G$, such that $b$ has weights $4^{2},-4^{2}, 0^{4}$ and $3,-3,1,-1,4^{2},-4^{2}$ on natural modules for the $D_{4}$ factors, and weights $4^{4},-4^{4}, 3,-3,1,-1,0^{4}$ on the natural $D_{8}$-module. Hence $C_{D_{8}}(b)^{0}=$ $A_{1} A_{1} A_{3} T_{3}$. Moreover, from the above description of $L(G) \downarrow A_{1}^{5}$ we calculate that $\operatorname{dim} C_{L(G)}(b)=24$, so it follows that $C_{G}(a)^{0}=A_{1} A_{1} A_{3} T_{3}$. Now $S<Q A_{1}^{4}<$ $A_{1} A_{1} A_{3}$. Hence $Q=1$ and $S<\bar{S}<A_{1}^{4}$, where $\bar{S} \cong S L_{2}(K)$ has all weights on $L(G)$ less than $q$.

Next consider $i_{1} \ldots i_{4}=0112$. If $S<Q A_{1}^{4}$ with the $A_{1}^{4}$ not in $D_{4}$, then the usual $T_{1}$ argument works. Otherwise, we find that $L(G) \downarrow S$ has no 3 -fold tensor factors, and has $1 \otimes 1^{(8)} / 1^{0}$; this is not possible, by 6.1 .

To complete the $k=4$ case, let $i_{1} \ldots i_{4}=0022$. The $T_{1}$ argument works if $S<Q A_{1}^{4}$ with $A_{1}^{4}<D_{4}$, so suppose this is not the case. If $A=B_{2}(16)$, then we work out $C_{G}(a)^{0}$ as above, and find that it is $A_{3} A_{3} T_{2}$. Then $S<\bar{S}<A_{1}^{4}<A_{3} A_{3}$, where $\bar{S}$ has all weights less than $q$. Now suppose $A=G_{2}(16)$. Here we find that

$$
L(G) \downarrow S=\left(1 \otimes 1^{(8)}\right)^{4} /\left(1^{(2)} \otimes 1^{(4)}\right)^{4} /\left(1 \otimes 1^{(4)}\right)^{16} /\left(1^{(4)}\right)^{24} /\left(1^{(8)}\right)^{6} /\left(1^{(2)}\right)^{6} / 1^{24} / 0^{32} .
$$

By 6.1 , the factors $\left(1 \otimes 1^{(8)}\right)^{4}$ must occur in the restriction of a composition factor $10 \otimes 10^{(8)}$ or $10 \otimes 01^{(4)}$ of $L(G) \downarrow A$.

Suppose there is a factor $10 \otimes 10^{(8)}$. This restricts to $S$ as $\left(1 \otimes 1^{(8)}\right)^{4} /\left(1^{(8)}\right)^{4} / 1^{4} / 0^{4}$. The remaining factors $\left(1 \otimes 1^{(4)}\right)^{16}$ in $L(G) \downarrow S$ must come from $\left(10 \otimes 10^{(4)}\right)^{4}$ in $L(G) \downarrow A$, and this restricts to $S$ as $\left(1 \otimes 1^{(4)}\right)^{16} / 1^{16} /\left(1^{(4)}\right)^{16} / 0^{16}$. Now the remaining factors $\left(1^{(2)} \otimes 1^{(4)}\right)^{4}$ must come from $10^{(2)} \otimes 10^{(4)}$, which restricts to $S$ as $\left(1^{(2)} \otimes 1^{(4)}\right)^{4} /\left(1^{(2)}\right)^{4} /\left(1^{(4)}\right)^{4} / 0^{4}$. Of the factors in $L(G) \downarrow S$, there remain $1^{4} /\left(1^{(2)}\right)^{2} /\left(1^{(4)}\right)^{4} /\left(1^{(8)}\right)^{2} / 0^{8}$, of total dimension 32 . These factors must come from composition factors of $L(G) \downarrow A$ which are twists of 10 (say $a$ of them), 01 (say $b$ of them) and 00. Since $10 \downarrow S=1^{2} / 0^{2}$ and $01 \downarrow S=1^{4} / 1^{(2)} / 0^{4}$ (see 6.1), counting 2-dimensional composition factors for $S$ gives $2 a+5 b=12$, while counting trivial composition factors yields $2 a+4 b=8$. Therefore $b=4$, forcing $a$ to be negative, a contradiction. This completes the argument when $L(G) \downarrow A$ has a composition 
factor $10 \otimes 10^{(8)}$. The other case, in which there is a composition factor $10 \otimes 01^{(4)}$, is handled by an entirely similar argument.

The case where $k=4$ is now settled.

Assume from now on that $k=5$. We have $A_{1}^{5}<A_{1} D_{4}$; take $1^{\left(2^{i_{1}}\right)}$ to be the projection of $S$ into the $A_{1}$ not lying in the $D_{4}$. Then either the usual $T_{1}$ argument works, or we can twist to take $i_{1} \ldots i_{5}$ to be one of the following (in some order):

$$
\text { 00022, 00112, 00122, 01112, 01122, 01222, } 00123 .
$$

Moreover, either the $T_{1}$ argument works, or we have $i_{1}=0$ in the second case, and $i_{1} \neq 2$ in the fourth case. Let $b$ be an element of order $q+1$ in $S$. In the following table we present some information for each of the above possibilities, which in almost all cases is sufficient to obtain a contradiction:

\begin{tabular}{lll}
$i_{1}, i_{2} \ldots i_{5}$ & some comp. factors of $L(G) \downarrow S$ & $C_{G}(b)^{\prime}$ \\
\hline 0,0022 & $\left(1^{(2)} \otimes 1^{(8)}\right)^{1} /\left(1 \otimes 1^{(8)}\right)^{2}$ & $A_{1} D_{4}$ \\
2,0002 & $\left(1 \otimes 1^{(2)} \otimes 1^{(4)}\right)^{1}$ & $A_{2} D_{4}$ \\
0,0112 & $\left(1 \otimes 1^{(2)} \otimes 1^{(4)}\right)^{4}$ & $A_{1} A_{4}$ or $A_{1}^{2} A_{2} A_{3}$ \\
0,0122 & $\left(1 \otimes 1^{(2)} \otimes 1^{(8)}\right)^{1} /\left(1^{(2)} \otimes 1^{(8)}\right)^{0}$ & \\
1,0022 & $\left(1 \otimes 1^{(2)} \otimes 1^{(4)}\right)^{8} /\left(1 \otimes 1^{(2)}\right)^{0}$ & $A_{2} D_{4}$ \\
2,0012 & $\left(1 \otimes 1^{(2)} \otimes 1^{(4)}\right)^{4}$ & $A_{1} A_{1} A_{3}$ \\
0,1112 & $\left(1 \otimes 1^{(2)} \otimes 1^{(4)}\right)^{6} /\left(1 \otimes 1^{(2)}\right)^{0}$ & $A_{2} D_{4}$ \\
1,0112 & $\left(1 \otimes 1^{(2)} \otimes 1^{(4)}\right)^{2}$ & $A_{1} A_{4}$ or $A_{1}^{2} A_{2} A_{3}$ \\
0,1122 & $\left(1 \otimes 1^{(2)} \otimes 1^{(4)}\right)^{8} /\left(1 \otimes 1^{(2)}\right)^{0}$ & $A_{1} D_{4}$ \\
1,0122 & $\left(1 \otimes 1^{(2)} \otimes 1^{(8)}\right)^{1} /\left(1 \otimes 1^{(8)}\right)^{0}$ & \\
2,0112 & $\left(1 \otimes 1^{(2)} \otimes 1^{(8)}\right)^{4}$ & $A_{2} A_{3}$ \\
0,1222 & $\left(1 \otimes 1^{(2)} \otimes 1^{(4)}\right)^{6}$ & $A_{2} D_{4}$ \\
1,0222 & $\left(1 \otimes 1^{(2)} \otimes 1^{(4)}\right)^{6}$ & $A_{2} D_{4}$ \\
2,0122 & $\left(1 \otimes 1^{(2)} \otimes 1^{(8)}\right)^{1}$ & $A_{1} A_{3}$ \\
0,0123 & $1 \otimes 1^{(2)} \otimes 1^{(4)} \otimes 1^{(8)}$ & \\
1,0023 & $\left(1^{(2)} \otimes 1^{(4)} \otimes 1^{(8)}\right)^{2}$ & $A_{2} A_{3}$ \\
2,0013 & $\left(1^{(2)} \otimes 1^{(4)} \otimes 1^{(8)}\right)^{2}$ & $A_{1} A_{3}$ \\
3,0012 & $\left(1^{(2)} \otimes 1^{(4)} \otimes 1^{(8)}\right)^{2}$ & $A_{1} A_{1} A_{3}$ \\
\hline
\end{tabular}

In each case, using 6.1 and 6.2 , we see that the information in the composition factors column rules out $A=G_{2}(16)$, and also rules out $A=B_{2}(16)$ in rows 4,10 and 15 of the table. Therefore $A=B_{2}(16)$ (and we are not in row 4, 10 or 15), and the element $b \in S$ is conjugate to $a$. We know that $S<Q A_{1}^{5}<C_{G}(a)^{\prime}$. This implies that $C_{G}(a)^{\prime}$ is $A_{1} D_{4}, A_{2} D_{4}$ or $A_{1}^{2} A_{2} A_{3}$; the last case is ruled out by 3.4, and in the first case, $S<\bar{S}<A_{1} D_{4}$ with $\bar{S} \cong S L_{2}(K)$ having all weights on $L(G)$ less than $q$. Thus $C_{G}(a)^{\prime}=A_{2} D_{4}$. Then $S<\left(Q A_{1}\right) A_{1}^{4}<A_{2} D_{4}$ with $Q A_{1}<A_{2}$ and $A_{1}^{4}<D_{4}$. If $i_{1}>0$ then using 1.2 we have $S<\bar{S}<A_{2} D_{4}$ with all weights of $\bar{S}$ less than $q$. The surviving cases are those in rows 7 and 12 of the table. For row 7 , twist to take $i_{1}, i_{2} \ldots i_{5}=3,0001$; then $S<\bar{S}<A_{2} D_{4}$, where $\bar{S}$ has highest weight 16 on $L(G)$, and has no composition factor of high weight 1 . The conclusion now follows from 1.4. For row 12, we twist to 2,3000 and apply the same argument.

To complete the proof of Theorem 1, it remains to handle case (iii) of 6.6 .

Lemma 6.15. Case (iii) of 6.6 does not hold if $q \geq 32$. 
Proof. Assume 6.6(iii) holds, so $S<Q A_{1}^{2} B_{1}^{2}<A_{1}\left(Q A_{1} B_{1}^{2}\right)<A_{1} D_{5}$. Moreover, $C_{G}(a)^{\prime}=A_{1} D_{5}$ : for otherwise by 6.5 and 6.7 , we are in a different case of 6.6 , already ruled out. Thus $C_{G}(a)^{0}=A_{1} D_{5} T_{2}$, of dimension 50 .

If $S$ projects trivially to one of the $B_{1}$ factors then $S<Q A_{1}^{4}$ (as $B_{1}<A_{1}^{2}$ ), contrary to 6.13 and 6.14 . Hence $S$ projects nontrivially to both the $B_{1}$ factors (and with distinct twists, by 6.6). Let the nontrivial projections of $S$ to the factors $A_{1}\left(A_{1} B_{1} B_{1}\right)<A_{1} D_{5}$ be $1^{\left(2^{i_{1}}\right)}, \ldots, 1^{\left(2^{i}{ }_{k}\right)}$, written in the order of the factors given (so $1^{\left(2^{i_{k-1}}\right)}, 1^{\left(2^{i} k\right)}$ are the projections to the $B_{1}$ factors). Take $i_{k-1}<i_{k}$.

If $k=2$, then the usual $T_{1}$ argument goes through, so assume $k \geq 3$. Also, if $k=4$ and $i_{1}, \ldots, i_{4}$ are all distinct, then by 6.8(ii), $L(G) \downarrow S$ has a composition factor $1^{\left(2^{i_{1}}\right)} \otimes \ldots \otimes 1^{\left(2^{i_{4}}\right)}$ but no composition factor $1^{\left(2^{i_{1}}\right)} \otimes 1^{\left(2^{i_{2}}\right)} \otimes 1^{\left(2^{i_{4}}\right)}$, contrary to $6.2(\mathrm{i}, \mathrm{ii})$.

Thus if $k=4$ then $i_{1}, \ldots, i_{4}$ are not all distinct. When $i_{r} \leq e-3$ for all $r$, the usual $T_{1}$ argument goes through. As $e \geq 5$ by hypothesis, we may therefore apply 6.4 to take one of the following to hold:

(1) $e=5$ and $i_{1} \ldots i_{k}=0013,0023,0113$ or 013 (in some order);

(2) $e=6$ and $i_{1} \ldots i_{k}=0024$ or 024 .

Consider first case (1). We have $S<A_{1}\left(Q A_{1} B_{1}^{2}\right)<A_{1} D_{5}$. Given $i_{1}, \ldots, i_{k}$, the composition factors of $L(G) \downarrow S$ can be worked out from the restrictions $L(G) \downarrow A_{1} D_{5}$ and $V_{D_{5}}\left(\lambda_{m}\right) \downarrow A_{1} B_{1}^{2}(m=1,4,5)$ given in the proof of 6.8(ii). From 6.8(ii), we see that if neither $i_{k-1}$ nor $i_{k}$ is 3 , then the usual $T_{1}$ argument goes through. Hence we may take it that $i_{k}=3$. In the table below, for each possibility for $\left(i_{1}, \ldots, i_{k}\right)$, we give various composition factors of $L(G) \downarrow S$ and their multiplicities; we also give the dimension of $C_{G}(b)$, where $b$ is an element of order $q+1$ in $S$. In the left hand column, a symbol "-" indicates a trivial projection.

\begin{tabular}{lll}
$\left(i_{1}, \ldots, i_{k}\right)$ & $L(G) \downarrow S$ & $\operatorname{dim} C_{G}(b)$ \\
\hline$(0,0,1,3)$ & $\left(1 \otimes 1^{(2)} \otimes 1^{(8)}\right)^{8} /\left(1 \otimes 1^{(8)}\right)^{0} / \ldots$ & 24 \\
$(0,1,0,3)$ & $\left(1 \otimes 1^{(2)} \otimes 1^{(8)}\right)^{4} /\left(1^{(2)} \otimes 1^{(16)}\right)^{3} /\left(1 \otimes 1^{(2)}\right)^{6} / 1^{8} / \ldots$ & 20 \\
$(1,0,0,3)$ & $\left(1 \otimes 1^{(2)} \otimes 1^{(8)}\right)^{4} /\left(1^{(2)} \otimes 1^{(16)}\right)^{3} /\left(1 \otimes 1^{(2)}\right)^{6} / 1^{8} / \ldots$ & 20 \\
$(0,0,2,3)$ & $\left(1 \otimes 1^{(4)} \otimes 1^{(8)}\right)^{8} /\left(1 \otimes 1^{(4)}\right)^{0} / \ldots$ & 24 \\
$(0,2,0,3)$ & $\left(1 \otimes 1^{(4)} \otimes 1^{(8)}\right)^{4} /\left(1^{(2)} \otimes 1^{(4)} \otimes 1^{(8)}\right)^{2} /$ & \\
& $\left(1^{(4)} \otimes 1^{(8)}\right)^{4} / \ldots$ & 16 \\
$(2,0,0,3)$ & $\left(1 \otimes 1^{(4)} \otimes 1^{(8)}\right)^{4} /\left(1^{(2)} \otimes 1^{(4)} \otimes 1^{(8)}\right)^{2} /$ & \\
& $\left(1^{(4)} \otimes 1^{(8)}\right)^{4} / \ldots$ & 16 \\
$(0,1,1,3)$ & $\left(1 \otimes 1^{(2)} \otimes 1^{(8)}\right)^{4} /\left(1 \otimes 1^{(4)} \otimes 1^{(8)}\right)^{2} /\left(1 \otimes 1^{(8)}\right)^{4} / \ldots$ & 16 \\
$(1,0,1,3)$ & $\left(1 \otimes 1^{(2)} \otimes 1^{(8)}\right)^{4} /\left(1 \otimes 1^{(4)} \otimes 1^{(8)}\right)^{2} /\left(1 \otimes 1^{(8)}\right)^{4} / \ldots$ & 16 \\
$(1,1,0,3)$ & $\left(1 \otimes 1^{(2)} \otimes 1^{(8)}\right)^{8} /\left(1 \otimes 1^{(2)}\right)^{0} / \ldots$ & 32 \\
$(0,-, 1,3)$ & $\left(1 \otimes 1^{(2)} \otimes 1^{(8)}\right)^{4} /\left(1 \otimes 1^{(2)}\right)^{0} / \ldots$ & 34 \\
$(-, 0,1,3)$ & $\left(1 \otimes 1^{(2)} \otimes 1^{(8)}\right)^{8} /\left(1 \otimes 1^{(2)}\right)^{0} / \ldots$ & 34 \\
$(1,-, 0,3)$ & $\left(1 \otimes 1^{(2)} \otimes 1^{(8)}\right)^{8} /\left(1 \otimes 1^{(2)}\right)^{0} / \ldots$ & 38 \\
$(-, 1,0,3)$ & $\left(1 \otimes 1^{(2)} \otimes 1^{(8)}\right)^{8} /\left(1 \otimes 1^{(2)}\right)^{0} / \ldots$ & 38 \\
\hline
\end{tabular}

If $A=B_{2}(q)$ then $S$ contains a conjugate $b$ of $a$; but $C_{G}(a)$ has dimension 50 , which conflicts with the dimension of $C_{G}(b)$ given in the table.

Therefore $A=A_{2}^{\epsilon}(q)$. By 6.2(ii,vi), the information given in the second column of the table rules out all possibilities for $\left(i_{1}, \ldots, i_{k}\right)$ except $(0,1,0,3)$ and $(1,0,0,3)$. 
In these cases we have

$$
L(G) \downarrow S=\left(1 \otimes 1^{(2)} \otimes 1^{(8)}\right)^{4} /\left(1^{(2)} \otimes 1^{(16)}\right)^{3} /\left(1 \otimes 1^{(2)}\right)^{6} / 1^{8} / \ldots
$$

The 3-fold tensor factors force $L(G) \downarrow A$ to have four composition factors of the form $\alpha \otimes \beta^{(2)} \otimes \gamma^{(8)}$ with $\alpha, \beta, \gamma \in\{10,01\}$. As $1^{(2)} \otimes 1^{(16)}$ occurs with odd multiplicity, it must occur as a composition factor in a self-dual factor of $L(G) \downarrow A$, which must therefore by 6.1 be $11 \otimes 11^{(8)}$. Now $\left(11 \otimes 11^{(8)}\right) \downarrow S$ and the four factors $\left(\alpha \otimes \beta^{(2)} \otimes \gamma^{(8)}\right) \downarrow S$ account for all eight composition factors 1 in $L(G) \downarrow S$. However, two of the composition factors $1 \otimes 1^{(2)}$ are still to be accounted for in other composition factors of $L(G) \downarrow A$. By 6.1, there is no irreducible $A$-module $V$ such that $V \downarrow S$ has $1 \otimes 1^{(2)}$ but not 1 , so this is impossible.

Now consider case (2), in which $e=6$ and $i_{1} \ldots i_{k}=0024$ or 024 . The usual $T_{1}$ argument works unless $i_{k}=4$. As above, we calculate the following information.

\begin{tabular}{lll}
$\left(i_{1}, \ldots, i_{k}\right)$ & $L(G) \downarrow S$ & $\operatorname{dim} C_{G}(b)$ \\
\hline$(0,0,2,4)$ & $\left(1 \otimes 1^{(4)} \otimes 1^{(16)}\right)^{8} /\left(1 \otimes 1^{(4)}\right)^{0} / \ldots$ & 20 \\
$(0,2,0,4)$ & $\left(1 \otimes 1^{(4)} \otimes 1^{(16)}\right)^{4} /\left(1^{(2)} \otimes 1^{(4)} \otimes 1^{(16)}\right)^{2} /$ & \\
& $\left(1^{(4) \otimes 1^{(16)}}\right)^{4} / \ldots$ & 16 \\
$(2,0,0,4)$ & $\left(1 \otimes 1^{(4)} \otimes 1^{(16)}\right)^{4} /\left(1^{(2)} \otimes 1^{(4)} \otimes 1^{(16)}\right)^{2} /$ & \\
& $\left(1^{(4) \otimes 1^{(16)}}\right)^{4} / \ldots$ & 16 \\
$(0,-, 2,4)$ & $\left(1 \otimes 1^{(4)} \otimes 1^{(16)}\right)^{8} /\left(1 \otimes 1^{(4)}\right)^{0} / \ldots$ & 34 \\
$(-, 0,2,4)$ & $\left(1 \otimes 1^{(4)} \otimes 1^{(16)}\right)^{8} /\left(1 \otimes 1^{(4)}\right)^{0} / \ldots$ & 34 \\
$(2,-, 0,4)$ & $\left(1 \otimes 1^{(4)} \otimes 1^{(16)}\right)^{8} /\left(1 \otimes 1^{(4)}\right)^{0} / \ldots$ & 34 \\
$(-, 2,0,4)$ & $\left(1 \otimes 1^{(4)} \otimes 1^{(16)}\right)^{8} /\left(1 \otimes 1^{(4)}\right)^{0} / \ldots$ & 34
\end{tabular}

Now we obtain a contradiction as before. This completes the proof of the lemma.

Lemma 6.16. Case (iii) of 6.6 does not hold if $q=16$.

Proof. Assume $q=16$ and 6.6(iii) holds. Then $A=B_{2}(16)$ or $G_{2}(16)$. As in the previous lemma, $\operatorname{dim} C_{G}(a)=50$.

Again let the nontrivial projections of $S$ to the factors $A_{1}\left(A_{1} B_{1} B_{1}\right)<A_{1} D_{5}$ be $1^{\left(2^{i_{1}}\right)}, \ldots, 1^{\left(2^{i_{k}}\right)}$. As in the previous lemma, the projections to the $B_{1}$ factors are nontrivial and distinct. Twisting if necessary, we can take $i_{1} i_{2} \in\{00,01,02,11,12$, $22,0-, 1-\}$ and $i_{3} i_{4} \in\{01,02,12\}$. Moreover, the $T_{1}$ argument goes through in all cases where $i_{3} i_{4}=01$, except when $i_{1} i_{2}=12$ or 22 .

For all the possibilities for $i_{1} \ldots i_{k}$, we calculate $L(G) \downarrow S$ and $\operatorname{dim} C_{G}(b)$ as in the previous lemma. In all cases we find that $\operatorname{dim} C_{G}(b)<50$, ruling out $A=B_{2}(16)$ in the usual way. Therefore $A=G_{2}(16)$. If $i_{3} i_{4} \neq 02$ or $i_{1} i_{2} \notin\{02,0-\}$, we find that $L(G) \downarrow S$ is either

$$
\left(1 \otimes 1^{(2)} \otimes 1^{(4)}\right)^{r} / \ldots . \text { with } r<8,
$$

or

$$
\left(1 \otimes 1^{(2)} \otimes 1^{(4)}\right)^{8} /\left(1^{\left(2^{i}\right)} \otimes 1^{\left(2^{j}\right)}\right)^{0} / \ldots \text { for some } i, j \in\{0,1,2\} .
$$

Both possibilities contradict 6.2(ii,iv).

Finally, consider the cases where $i_{3} i_{4}=02$ and $i_{1} i_{2}=02$ or $0-$. Assume first that $\left(i_{1}, i_{2}, i_{3}, i_{4}\right)=(0,2,0,2)$. Then we find that

$$
L(G) \downarrow S=\left(1 \otimes 1^{(2)}\right)^{2} /\left(1 \otimes 1^{(4)}\right)^{12} /\left(1 \otimes 1^{(8)}\right)^{6} /\left(1^{(2)} \otimes 1^{(8)}\right)^{3} /\left(1^{(4)} \otimes 1^{(8)}\right)^{2} / \ldots
$$


The composition factor $1^{(2)} \otimes 1^{(8)}$ appears with odd multiplicity. By 6.1 , the only way this can happen is if $L(G) \downarrow A$ has a composition factor $01 \otimes 01^{(4)}$ (of dimension 196). However, $\left(01 \otimes 01^{(4)}\right) \downarrow S$ has $1 \otimes 1^{(4)}$ with multiplicity 16 , whereas it only occurs with multiplicity 12 in $L(G) \downarrow S$, a contradiction. Similar contradictions are reached for the other possibilities for $\left(i_{1}, \ldots, i_{4}\right)$.

This completes the proof of Theorem 1 .

\section{Proof of Theorem 4}

Let $G$ be a simple algebraic group over the algebraically closed field $K$ of characteristic $p$. Let $X=X(q)<G$ with $q=p^{e}$ and $X$ not of the same type as $G$, and suppose that $X$ is irreducible on each $G$-composition factor of $L(G)$. We aim to show that $X$ and $G$ are as in the conclusion of Theorem 4. We may as well assume that $G$ is of adjoint type; as $X$ is irreducible on each $G$-composition factor of $L(G)$, we then have $Z(X)=Z(G)=1$. The $G$-composition factors of $L(G)$ are given by 1.10 .

We now embark upon the proof of Theorem 4. We first dispose of classical groups.

Lemma 7.1. Theorem 4 holds if $G$ is classical.

Proof. First observe that $X$ does not lie in a parabolic subgroup of $G$ : for if $X<P$, a parabolic, then $X$ leaves invariant the series $0<L\left(R_{u}(P)\right)<L(P)<L(G)$, hence cannot be irreducible on all nontrivial $G$-composition factors of $L(G)$, by 1.10 . We now apply [ST1, Theorem 1]; this implies that $X$ lies in a closed, connected, simple subgroup $\bar{X}$ of $G$ of the same type as $X$. Then $X$ fixes $L(\bar{X})$.

We now claim that $\operatorname{dim} \bar{X}<\operatorname{dim} G-2$. For suppose $\operatorname{dim} \bar{X} \geq \operatorname{dim} G-2$. If a maximal unipotent subgroup of $\bar{X}$ is also one of $G$, then $\bar{X}=G$ (see [Se4, 1.6] for instance), which is not the case. The dimension of a simple algebraic group is equal to the rank of the group plus twice the dimension of a maximal unipotent subgroup; it follows that $\bar{X}$ is a subgroup of maximal rank in $G$, and that a maximal unipotent subgroup of $\bar{X}$ has dimension just one less than a maximal unipotent subgroup of $G$. It is trivial to check that this cannot happen.

It follows from the previous paragraph and 1.10 that $(G, p)$ must be $\left(B_{n}, 2\right)$ or $\left(C_{n}, 2\right)$. Moreover, $\bar{X}$ is irreducible on $V_{G}\left(\lambda_{1}\right)$ and $V_{G}\left(\lambda_{2}\right)$. At this point [Se1, Theorem 1] determines all possibilities for $\bar{X}$ : either $\bar{X}=D_{n}$, or $n=3$ and $\bar{X}=G_{2}$, as in the conclusion of Theorem 4.

In view of 7.1, we assume from now on that $G$ is of exceptional type.

Let $V$ be a nontrivial $G$-composition factor of $L(G)$, so that $X$ is irreducible on $V$. Write $V=V_{G}(\lambda)$ with $\lambda$ as in 1.10 (up to twists).

If $P_{X}=Q_{X} L_{X}$ is a parabolic subgroup of $X$ with unipotent radical $Q_{X}$ and Levi subgroup $L_{X}$, then by [BT], there is a parabolic subgroup $P=Q L$ of $G$ containing $P_{X}$ and such that $Q_{X} \leq Q$, the unipotent radical of $P$. The next result follows from the argument in [ST1, p.565] (third paragraph).

Lemma 7.2. We have $C_{V}\left(Q_{X}\right)=C_{V}(Q)$, and $P_{X}$ and $P$ are both irreducible on this space. The high weight of $C_{V}(Q)$ as an $L$-module is the restriction of $\lambda$ to $L$. Assuming this action is nontrivial, we have $\lambda=\lambda_{i}$ for some $i$, and if $L_{0}$ is the simple factor of $L$ involving the root $\alpha_{i}$, then the possibilities for $C_{V}(Q) \downarrow L_{0}$ are 
as in Table 5. Moreover, if $\left(G, L_{0}\right)=\left(E_{7}, E_{6}\right)$ or $\left(E_{6}, D_{5}\right)$, then $Q$ and $Q_{X}$ are abelian.

\section{TABLE 5}

\begin{tabular}{lll}
$G$ & $L_{0}$ & $C_{V}(Q) \downarrow L_{0}$ (up to auts. of $\left.L_{0}\right)$ \\
\hline$E_{8}$ & $D_{7}$ or $A_{r}(r \leq 7)$ & $V_{L_{0}}\left(\lambda_{1}\right)$ \\
$E_{7}$ & $E_{6}, D_{5}$ or $A_{r}(r \leq 6)$ & $V_{L_{0}}\left(\lambda_{1}\right)$ \\
$E_{6}$ & $D_{5}, D_{4}$ & $V_{L_{0}}\left(\lambda_{4}\right)$ \\
& $A_{r}(r \leq 4)$ & $V_{L_{0}}\left(\lambda_{1}\right)$ \\
$F_{4}$ & $B_{3}, C_{3}(p=2)$, or $A_{r}(r \leq 2)$ & $V_{L_{0}}\left(\lambda_{1}\right)$ \\
\hline
\end{tabular}

Lemma 7.3. $X$ is not $A_{1}(q)$.

Proof. Suppose $X=A_{1}(q)$, and let $t$ be a generator for a Cartan subgroup of $X$. By the representation theory of $X, C_{V}(t)$ has dimension 1 unless $V$ is the Steinberg module for $X$; the latter is impossible as $\operatorname{dim} V$ cannot be equal to $q$ (see 1.10). However, $t$ lies in a maximal torus of $G$, so $C_{V}(t)$ must certainly be bigger than 1 , which is a contradiction.

Lemma 7.4. $X$ is not $A_{2}^{\epsilon}(q)$.

Proof. Suppose that $X=A_{2}^{\epsilon}(q)$. Now $V$ is a self-dual $X$-module, so by [ST1], $V$ lifts to a self-dual $A_{2}(K)$-module. This is a tensor product of twists of modules of the form $V_{A_{2}}(a a)$, where $0 \leq a \leq p-1$.

Now by 1.8 , either $W_{X}(a a)=V_{X}(a a)$, of dimension $(a+1)^{3}$, or $W_{X}(a a)$ has two composition factors, namely $V_{X}(a a)$ and $V_{X}(p-a-2, p-a-2)$; in the latter case, $2 a+2>p \geq a+2$ and $V_{X}(p-a-2, p-a-2)=W_{X}(p-a-2, p-a-2)$, whence $\operatorname{dim} V_{X}(a a)=(a+1)^{3}-(p-a-1)^{3}$.

If $p=2$ then $V$ is a tensor product of twists of $V_{X}(11)$, which has dimension 8; but from 1.10 we see that $\operatorname{dim} V$ is not a power of 8 . Hence $p>2$.

Suppose first that $G=E_{8}$. Then $\operatorname{dim} V=248=8.31$, so there is a restricted module $V_{X}(a a)$ of dimension $2^{r} .31(r \leq 3)$. Therefore $2^{r} .31$ must be equal to $(a+1)^{3}-(p-a-1)^{3}$. This has a factor $2 a+2-p$, which, since $p$ is odd, forces $2 a+2-p=1$. Then $2^{r} .31=(a+1)^{3}-a^{3}$, which is not possible.

Now let $G=E_{7}$. Then $\operatorname{dim} V=133=7.19($ recall $p \neq 2)$. As 133 is not a difference of cubes, we must have $V \downarrow X \cong V_{X}(a a) \otimes V_{X}(b b)^{\left(p^{i}\right)}$ with $(a+1)^{3}-$ $(p-a-1)^{3}=7$ and $(b+1)^{3}-(p-b-1)^{3}=19$. This is impossible.

Next consider $G=E_{6}$ or $F_{4}$. Here $\operatorname{dim} V=52,77$ or 78 , none of which is the product of a difference of cubes.

Finally, if $G=G_{2}$, then as 14 is not a product of differences of cubes, we must have $p=3$ and $\operatorname{dim} V=7$. Subgroups $A_{2}^{\epsilon}(q)$ of $G_{2}$ are identified by [LST, Theorem 2]: they lie in maximal rank connected subgroups $A_{2}$. However these are not irreducible on both $V_{G}\left(\lambda_{1}\right)$ and $V_{G}\left(\lambda_{2}\right)$, so no examples arise here.

Lemma 7.5. Theorem 4 holds if $G=G_{2}$.

Proof. Suppose $G=G_{2}$. By $7.3, \operatorname{rk}(X) \geq 2$ (recall that $\operatorname{rk}(X)$ denotes the rank of the simple algebraic group corresponding to $X)$.

Since a parabolic of $X$ lies in a parabolic of $G$ (by [BT]), we deduce that $X$ is of type $A_{2}^{\epsilon}(q), B_{2}(q), G_{2}(q),{ }^{3} D_{4}(q)$ or ${ }^{2} A_{3}(q)$. The first case is out by 7.4 , and 
$X \neq G_{2}(q)$ as $X$ is assumed not to have the same type as $G$; also $X \neq{ }^{3} D_{4}(q)$ since ${ }^{3} D_{4}(q)$ has no nontrivial representation of dimension 7 . By the argument in [ST1, p.565] (third paragraph), up to twists, $V \downarrow X$ is 10,01 or 11 if $X=B_{2}(q)$, and is 101 or 010 if $X={ }^{2} A_{3}(q)$. As $\operatorname{dim} V=14-7 \delta_{p, 3}$, the only possibility is that $X={ }^{2} A_{3}(q), V \downarrow X=101$ with $p=2$. But here $X$ has a parabolic subgroup $Q_{X} L_{X}$ with $L_{X}^{\prime} \cong A_{1}\left(q^{2}\right)$ acting on $C_{V}\left(Q_{X}\right)$ as $1 \otimes 1^{(q)}$, which does not agree with the action of a parabolic of $G$ containing $Q_{X} L_{X}$.

We assume from now on that $G \neq G_{2}$.

Lemma 7.6. $X$ is not $B_{2}^{\epsilon}(q)$ or $G_{2}^{\epsilon}(q)$.

Proof. Suppose that $X=B_{2}^{\epsilon}(q)$ or $G_{2}^{\epsilon}(q)$, and let $V \downarrow X \cong V_{X}(a b) \cong V_{X}\left(a_{0} b_{0}\right) \otimes \ldots$ with $a_{0}, b_{0} \leq p-1$. At several points in the proof we make use of the sum formula in [An], which can be used to calculate the dimensions of many modules $V_{X}(c d)$ with $c, d$ reasonably small.

First assume $p=2$ or 3 . By [GS] for $X=G_{2}^{\epsilon}(q)$, and by the sum formula [An] for $X=B_{2}^{\epsilon}(q)$, the possibilities for $\operatorname{dim} V_{X}\left(a_{0} b_{0}\right)$ are as follows:

\begin{tabular}{llll}
$X$ & $p$ & $a_{0} b_{0}$ & $\operatorname{dim} V_{X}\left(a_{0} b_{0}\right)$ \\
\hline$B_{2}^{\epsilon}(q)$ & 2 & $10,01,11$ & $4,4,16$ (resp.) \\
$G_{2}(q)$ & 2 & $10,01,11$ & $6,14,64$ \\
$B_{2}(q)$ & 3 & $10,20,01,11,21,02,12,22$ & $5,14,4,16,40,10,25,81$ \\
$G_{2}^{\epsilon}(q)$ & 3 & $10,20,01,11,21,02,12,22$ & $7,27,7,49,189,27,189,729$ \\
\hline
\end{tabular}

As $\operatorname{dim} V$ is a product of dimensions in the table, we have a contradiction.

Thus $p>3$. At this point we make use of 7.2. Take a parabolic subgroup $P_{X}=Q_{X} L_{X}$ of $X$, with $L_{X}^{\prime} \cong A_{1}(q)$. Then $P_{X}$ lies in a parabolic subgroup $Q L$ of $G$ with $Q_{X} \leq Q$, and $L_{X}$ is irreducible on the space $C_{V}(Q)$, which is given as $L$-module in Table 5 . We can choose $P_{X}$ so that $C_{V}(Q) \downarrow L_{X} \cong V_{L_{X}}(a)$ or $V_{L_{X}}(b)$.

We claim that one of the following holds:

$$
\begin{array}{ll}
G=E_{8}: & a_{0}, b_{0} \leq 7, \\
G=E_{7}: & a_{0}, b_{0} \leq 6, \\
G=E_{6}: & a_{0}, b_{0} \leq 4, \\
G=F_{4}: & a_{0}, b_{0} \leq 6 .
\end{array}
$$

To see this we argue as follows. If the claim is false, then from Table 5 we see that $L_{0}=D_{7}, E_{6}, D_{5}, D_{5}$ or $D_{4}$ and $L_{X}^{\prime}$ is irreducible on $V_{L_{0}}(\lambda)$, where $\lambda=\lambda_{1}, \lambda_{1}, \lambda_{1}, \lambda_{4}$ or $\lambda_{4}$, respectively. This is impossible for $V_{D_{r}}\left(\lambda_{1}\right)$ and $V_{D_{4}}\left(\lambda_{4}\right)$, by 1.1 , and for $V_{D_{5}}\left(\lambda_{4}\right)$, by [LS2, 2.13]. Finally, if $L_{0}=E_{6}$ then $X \neq G_{2}(q)$ since $Q_{X}$ is abelian by 7.2 , so $X=B_{2}(q)$. We shall see in the next paragraph that $V_{X}(a b)$ is restricted; hence $a$ or $b$ must be 26 . But it is easy to see that this forces $\operatorname{dim} V>\operatorname{dim} L(G)=$ 133 , a contradiction. This establishes the claim.

As $p>3, \operatorname{dim} V$ is $248,133,78$ or 52 . The irreducible $K X$-modules of dimension 8 or less, dividing $\operatorname{dim} V$, are as follows:

$$
\begin{array}{ll}
X=B_{2}(q): & V_{X}(01) \text { of dimension } 4 \\
X=G_{2}(q): & V_{X}(10) \text { of dimension } 7 .
\end{array}
$$


Hence, if $V_{X}(a b)$ is not restricted, then it is a tensor product of one of the above modules with a twist of a restricted module $V_{X}(c d)$; the latter module has dimension 62 or 13 (for $X=B_{2}(q), G=E_{8}$ or $F_{4}$ ), or dimension 19 (for $X=G_{2}(q), G=E_{7}$ ). When $X=G_{2}(q), G=E_{7}$, we have $C_{V}(Q) \downarrow L_{X} \cong V_{L_{X}}(1) \otimes V_{L_{X}}(c)^{\left(p^{i}\right)}$ or $V_{L_{X}}(d)^{\left(p^{i}\right)}$, whence $c \leq 2$ and $d \leq 6$ by 7.2 . It is easy to see that no such module $V_{X}(c d)$ has dimension 19: use [GS] for the small weights, and count conjugates for the others. A similar argument applies when $X=B_{2}(q)$. Here $c \leq 7, d \leq 3$. Moreover, since $Z(X)=1$ and $V \downarrow X \cong 01 \otimes c d^{\left(p^{i}\right)}$, the module $V(c d)$ must admit the action of the simply connected group of type $B_{2}$, and hence $d$ must be odd; in particular, the central element -1 has determinant 1 on $V(c d)$, so $\operatorname{dim} V(c d)$ is even, hence equal to 62 . Now [Pr] shows that all weights of the Weyl module $W_{B_{2}}(c d)$ appear as weights of $V_{B_{2}}(c d)$, and counting conjugates of such weights shows that the only possibilities with $\operatorname{dim} V_{B_{2}}(c d) \leq 62$ are among the following:

$$
c d=01,03,11,13,21,23,31,41 .
$$

The dimensions of these modules can be calculated using the sum formula in [An]; we find that none of them is equal to 62 .

We conclude that $V_{X}(a b)$ is restricted (that is, $a=a_{0}, b=b_{0}$ ). Now suppose $X=B_{2}(q)$. As $Z(X)=1, b$ is even. The dimensions of $V_{X}(a b)$ with $a, b \leq 7$ can be calculated using the sum formula in $[\mathrm{An}]$, and we find that the only cases where this dimension is less than or equal to 248 are as follows:

\begin{tabular}{ll}
$a b$ & $\operatorname{dim} V_{B_{2}}(a b)$ \\
\hline 06 & 84 \\
16 & 231 \\
04 & 35 \\
14 & 105 \\
24 & $220-105 \delta_{p, 5}-71 \delta_{p, 7}$ \\
02 & 10 \\
12 & 35 \\
22 & $81-13 \delta_{p, 5}-10 \delta_{p, 7}$ \\
32 & $154-68 \delta_{p, 5}$ \\
42 & $260-54 \delta_{p, 7}-81 \delta_{p, 11}$ \\
52 & $199(p=7)$ \\
10 & 5 \\
20 & $14-\delta_{p, 5}$ \\
30 & $30-5 \delta_{p, 7}$ \\
40 & $55-\delta_{p, 7}$ \\
50 & $91-30 \delta_{p, 11}$ \\
60 & $140-14 \delta_{p, 11}-55 \delta_{p, 13}$ \\
70 & $204-5 \delta_{p, 11}-30 \delta_{p, 13}$ \\
\hline
\end{tabular}

None of these dimensions is equal to $\operatorname{dim} V$, a contradiction.

Now let $X=G_{2}(q)$, and recall that $p>3$. Again, application of the sum formula shows that the only cases where $V_{X}(a b)$ (with $a, b \leq 7$ ) has dimension at most 248 
are:

\begin{tabular}{ll}
$a b$ & $\operatorname{dim} V_{G_{2}}(a b)$ \\
\hline 40 & $182-27 \delta_{p, 11}$ \\
30 & 77 \\
21 & $189-64 \delta_{p, 11}$ \\
20 & $27-\delta_{p, 7}$ \\
13 & $244(p=13)$ \\
12 & $286-189 \delta_{p, 5}-38 \delta_{p, 7}$ \\
11 & $64-26 \delta_{p, 7}$ \\
10 & 7 \\
03 & $273-77 \delta_{p, 5}-125 \delta_{p, 11}$ \\
02 & 77 \\
01 & 14 \\
\hline
\end{tabular}

The only possibility where $\operatorname{dim} V_{X}(a b)=\operatorname{dim} V$ is

$$
G=E_{8}, p=7 \text { and } V \downarrow X \cong V_{X}(12) \text {. }
$$

In this case, let $\alpha, \beta$ be fundamental roots for a $G_{2}$ root system, and let $t=$ $h_{\alpha}(-1) \in X$. We calculate $\operatorname{dim} C_{V}(t)$, where $V=V_{X}(12)$. (We shall then obtain a contradiction by comparing this with the known possibilities for $\operatorname{dim} C_{L(G)}(t)$.) All the weights appearing in $V$ and their multiplicities are listed in [GS]. The dominant weights which are subdominant to 12 are

$$
12,40,21,02,30,11,20,01,10,00
$$

and these appear in $V_{X}(12)$ with multiplicities $1,1,2,2,3,4,6,6,8,8$, respectively. The orbits of these weights under the Weyl group of $X$ are as follows:

$$
\begin{array}{ll}
\text { orbit of } 12: & 12,1(-3), 8(-3), 8(-5), 7(-5), 7(-2) \text { and negatives } \\
\text { orbit of } 40: & 40,4(-4), 8(-4) \text { and negatives } \\
\text { orbit of } 21: & 21,2(-3), 7(-3), 7(-4), 5(-4), 5(-1) \text { and negatives } \\
\text { orbit of } 02: & 02,6(-2), 6(-4) \text { and negatives } \\
\text { orbit of } 30: & 30,3(-3), 6(-3) \text { and negatives } \\
\text { orbit of } 11: & 11,1(-2), 5(-2), 5(-3), 4(-3), 4(-1) \text { and negatives } \\
\text { orbit of } 20: & 20,2(-2), 4(-2) \text { and negatives } \\
\text { orbit of } 01: & 01,3(-1), 3(-2) \text { and negatives } \\
\text { orbit of } 10: & 10,1(-1), 2(-1) \text { and negatives }
\end{array}
$$

The dimension of $C_{V}(t)$ is equal to the number of weights appearing in $V$ with first coordinate even. Hence we find $\operatorname{dim} C_{V}(t)=124$. But $C_{G}(t)$ is $D_{8}$ or $A_{1} E_{7}$, whence $\operatorname{dim} C_{L(G)}(t)=\operatorname{dim} C_{G}(t)=120$ or 136 , a contradiction. This completes the proof of the lemma.

Lemma 7.7. Theorem 4 holds if $G=F_{4}$.

Proof. Let $G=F_{4}$. By 7.3, 7.4 and 7.6, $\operatorname{rk}(X) \geq 3$. First suppose that $q>2$. Then by [LST, Theorem 2] there is a connected simple subgroup $\bar{X}$ of $G$ containing $X$ and of the same type as $X$. As $X$ fixes $L(\bar{X})$, we deduce from 1.10 that $L(\bar{X})$ has all its $\bar{X}$-composition factors of dimension $52-26 \delta_{p, 2}$. By 1.10 , there is no such group $\bar{X}$.

Now let $q=2$. The only possible subgroups $X$ having an irreducible module of dimension 26 are $D_{4}^{\epsilon}(2)$ and $B_{4}(2) \cong C_{4}(2)$, the unique such module being $V_{X}\left(\lambda_{2}\right)$. It is enough to rule out $X=D_{4}^{\epsilon}(2)$ (since $B_{4}(2)$ contains $D_{4}(2)$ acting irreducibly 
on $V\left(\lambda_{2}\right)$ ). So suppose $X=D_{4}^{\epsilon}(2)$ and pick a parabolic subgroup $P_{X}$ of $X$ of type $Q_{X} D_{3}^{\epsilon}(2)$ if $\epsilon= \pm$, and of type $Q_{X} L_{2}(8)$ if $\epsilon=3$. By [BT], $P_{X}<Q L$, a parabolic of $G$. If $\epsilon= \pm$, this must be a $B_{3^{-}}$or $C_{3}$-parabolic. Moreover, by $7.2, L$ must act irreducibly and nontrivially on $C_{V}(Q)=C_{V}\left(Q_{X}\right)$, for $V=V_{G}\left(\lambda_{i}\right), i=1,4$. But this is not the case, as $L$ in fact acts trivially on $C_{V}(Q)$ for one of these values of $i$.

Finally, suppose $\epsilon=3$. Then the factor $L_{2}(8)$ acts trivially on $C_{V}\left(Q_{X}\right)$ (where $\left.V=V_{X}\left(\lambda_{2}\right)\right)$, so $Q L$ is a $B_{2^{-}}$or $A_{1}$-parabolic as it must be trivial on both 26dimensional composition factors of $L(G)$. The 8-dimensional module $V_{X}\left(\lambda_{1}\right)$ restricts to $L_{2}(8)$ as $1^{(2)} \otimes 1^{(4)} / 1 / 1$, hence $V_{X}\left(\lambda_{2}\right) \downarrow L_{2}(8)$ has a composition factor $1 \otimes 1^{(2)} \otimes 1^{(4)}$. But on restriction to $Q L$, the modules $V_{G}\left(\lambda_{i}\right)(i=1,4)$ have all composition factors of dimension at most 5 , which is a contradiction.

From now on, we assume that $G=E_{6}, E_{7}$ or $E_{8}$. Also, by $7.3-7.6, \operatorname{rk}(X) \geq 3$.

Lemma 7.8. Let $L_{0}$ be a connected simple group of type $A_{r}(r \leq 7), D_{4}, D_{5}, D_{5}$, $D_{7}$ or $E_{6}$, and let $W=V_{L_{0}}(\mu)$, where $\mu=\lambda_{1}, \lambda_{1}, \lambda_{1}, \lambda_{4}, \lambda_{1}$ or $\lambda_{1}$, respectively. Suppose that $Y=Y(q)$ is a quasisimple subgroup of $L_{0}$ such that $\operatorname{rk}(Y) \geq 2$, $Y \neq G_{2}^{\epsilon}(q)$ and $Y$ is irreducible on $W$. Then one of the following holds (where $V \downarrow Y$ is given up to twists):

\begin{tabular}{|c|c|c|}
\hline$Y$ & $\nu$, where $V \downarrow Y=V_{Y}(\nu)$ & $L_{0}$ \\
\hline \multirow[t]{2}{*}{$\overline{A_{2}^{\epsilon}(q)}$} & $10,01,20,02,11,22$, & $\begin{array}{l}A_{2}, A_{2}, A_{5}, A_{5}, A_{7-\delta_{p, 3}} \\
\text { or } D_{4}, E_{6},\end{array}$ \\
\hline & $\begin{array}{l}\alpha \otimes \beta^{\left(p^{i}\right)} \otimes \gamma^{\left(p^{j}\right)} \\
(\alpha, \beta, \gamma \in\{10,01\})\end{array}$ & $E_{6}($ resp. $)$ \\
\hline$B_{2}^{\epsilon}(q)$ & $\begin{array}{l}10,01,02,20,11,01 \otimes 01^{\left(p^{i}\right)} \\
10 \otimes 10^{\left(2^{i}\right)}, 10 \otimes 01^{\left(2^{i}\right)}(p=2)\end{array}$ & $\begin{array}{l}A_{4-\delta_{p, 2}}, A_{3}, D_{5}, D_{7}, D_{5}, D_{5}, \\
D_{5}, D_{5} \text { (resp.) }\end{array}$ \\
\hline$A_{3}^{\epsilon}(q)$ & $100,001,010$ & $A_{3}, A_{3}, A_{5}$ \\
\hline$B_{3}(q)$ & $100,200(p \neq 7), 001$ & $A_{6-\delta_{p, 2}}, E_{6}, A_{7}$ or $D_{4}$ \\
\hline$C_{3}(q)(q$ odd $)$ & $100,010(p \neq 3)$ & $A_{5}, D_{7}$ \\
\hline$B_{4}(q)(q$ odd $)$ & 0001 & $D_{5}$ \\
\hline$C_{4}(q)$ & $1000,0100,0001(p=2)$ & $A_{7}, E_{6}, D_{5}$ \\
\hline$D_{4}^{\epsilon}(q)$ & $1000,0010,0001$ & $A_{7}$ or $D_{4}$ \\
\hline same type as $L_{0}$ & $\mu$ & $L_{0}$ \\
\hline
\end{tabular}

Proof. The dimension of $W$ is $r+1,8,10,16,14$ or 27 in the respective cases for $L_{0}$. The $Y$-modules of dimension up to 10 (self-dual if the dimension is 9 or 10) are well known (cf. [Li, 1.1]), and all possibilities are in the table. So assume $\operatorname{dim} W=14,16$ or 27 . Let $W=V_{Y}(\lambda)$. If this is a nontrivial tensor product, then the tensor factors have dimension at most 9 and either $W$ is in the table or $Y=A_{3}^{\epsilon}(q), L_{0}=D_{5}, \lambda=\lambda_{4}$ and $W \downarrow Y=\alpha \otimes \beta^{\left(p^{i}\right)}$ with $\alpha, \beta \in\{100,001\}$; however, in the latter case, $V_{D_{5}}\left(\lambda_{1}\right) \downarrow Y$ must be either $100 / 001 / 000^{2}$ or $010 / 000^{4}$, and in neither case does the spin module $V_{D_{5}}\left(\lambda_{4}\right)$ restrict irreducibly to $Y$, so this case does not occur. Now assume $V_{Y}(\lambda)$ is not a nontrivial tensor product; then we may assume $\lambda$ is restricted.

Suppose $Y$ is not of type $L_{0}$. When $Y=A_{2}^{\epsilon}(q), 1.8$ shows that the only possibility is $\lambda=22$ (giving $\operatorname{dim} V_{Y}(\lambda)=27$ ). For other types, we argue by counting conjugates of subdominant weights appearing in $V_{Y}(\lambda)$ that the only possibilities for $(Y, \lambda)$, 
with $V_{Y}(\lambda)$ having dimension between 11 and 27, are among the following:

\begin{tabular}{ll}
$Y$ & $\lambda$ \\
\hline$B_{2}(q)$ & $20,30,03,11,12$ \\
$B_{3}(q)$ & 200,002 \\
$C_{3}(q)$ & $200,010,001$ \\
$B_{4}(q)$ & 0100,0001 \\
$C_{4}(q)$ & 0100
\end{tabular}

For $Y=B_{2}(q)$ the tables in the proof of 7.6 exclude all but $\lambda=20,11$; for the other cases, $[\mathrm{BW}]$ excludes all but $(Y, \lambda)=\left(B_{3}(q), 200\right),\left(C_{3}(q), 010\right.$ or 001$),\left(B_{4}(q), 0001\right)$ and $\left(C_{4}(q), 0100\right)$. All of these except $\left(C_{3}(q), 001\right)$ ( $q$ odd) are in the conclusion; in the exceptional case, the 14-dimensional module $V_{C_{3}}(001)$ is symplectic rather than orthogonal. Note also that $p \neq 7,3$ for the $B_{3}(q), C_{3}(q)$ cases in the table, respectively, as otherwise $V_{Y}(\lambda)$ does not have the correct dimension.

Lemma 7.9. We have $V \cong V_{X}(\delta)$, where $\delta$ is as in Table 6 (given up to twists).

\section{TABLE 6}

\begin{tabular}{ll}
$X$ & $\delta$ \\
\hline$A_{3}(q)$ & $111,202,222$ \\
${ }^{2} A_{3}(q)$ & $0 a 0(a \leq 7), 1 a 1(a \leq 5)$ \\
${ }^{2} A_{4}(q)$ & 0110,1111 \\
$B_{3}(q)$ & $011,020,110,111,102,202,220$ \\
$C_{3}(q)$ & $002,020,011,101,111,202,220,110(p=2)$ \\
${ }^{2} D_{4}(q)$ & $2010,2001,1100$ \\
${ }^{3} D_{4}(q)$ & $a 000,0 b 00,1 c 00(a \leq 4, b \leq 3, c \leq 3), 2100$, \\
& $1010,1110,2010,3010,3110$ \\
& $1 d 10,1 d 11,2 d 10(p \leq 3, d \leq p-1)$ \\
$F_{4}(q)$ & 2000,0010 \\
${ }^{2} F_{4}(q)$ & 0100,1100 \\
\hline
\end{tabular}

Proof. Recall that $V$ is a self-dual $G$-module. We show first that if $V \downarrow X=V_{X}(\delta)$, then the weight $\delta$ is restricted. If not, then $V \downarrow X$ is a nontrivial tensor product. As $\operatorname{dim} V$ is $78-\delta_{p, 3}, 133-\delta_{p, 2}$ or 248 , we see that one of the tensor factors must have dimension $x \leq 8$. Thus $X$ has a self-dual irreducible $V_{X}(\chi)$ of dimension $x$, from which we deduce that $(X, \chi, x)=\left(A_{3}^{\epsilon}(q), 010,6\right),\left(B_{3}(q), 100,7\right),\left(B_{3}(q), 001,8\right)$, $\left(C_{3}(q), 100,6\right)\left(C_{4}(q), \lambda_{1}, 8\right)$ or $\left(D_{4}^{\epsilon}(q), \lambda_{i}, 8\right)(i=1,3,4)$. We have $V \downarrow X=\chi \otimes \gamma^{(r)}$, where $y=\operatorname{dim} V_{X}(\gamma)$ is 13 or 22,11 or $19,31,13$ or 22,31 , or 31 , respectively. Excluding the cases $(X, y)=\left(A_{3}^{\epsilon}(q), 22\right)$ or $\left(B_{3}(q), 31\right)$, we see in the usual way (counting conjugates of subdominant weights, etc.) that the only possibility for a self dual $X$-module of dimension $y$ is $X=C_{3}(q), \gamma=\lambda_{2}$ with $p=3$ and $y=13$; but then $x y=78$, whereas $\operatorname{dim} V=77$ for $G=E_{6}, p=3$. For the excluded case with $X=A_{3}^{\epsilon}(q)$, we have $x y=132$, so $G=E_{7}, p=2$. The only possibility for $\gamma$ is 111 ; but it is easy to see by counting conjugates of subdominant weights that $V_{X}(111)$ has dimension greater than 22 . The other excluded case with $X=B_{3}(q)$ is handled by counting conjugates of dominant weights in the usual way. Thus the weight $\delta$ is restricted. 
Consider first $X=A_{3}(q)$, and let $\delta=a b a$. Choose a parabolic $P_{X}=Q_{X} L_{X}$ of $X$ such that $L_{X}^{\prime} \cong A_{2}(q)$ and $C_{V}\left(Q_{X}\right) \downarrow L_{X} \cong V_{L_{X}}(a b)$. Then from 7.2 and 7.8, we see that $a b$ is as in the conclusion of 7.8 for $A_{2}(q)$, and so

$$
a b=00,10,01,20,02,11 \text {, or } 22 .
$$

Therefore $\delta=101,010,202,020,111$ or 222 . Clearly 101,010 and 020 have dimension less than 77, leaving the possibilities in Table 6 for $X=A_{3}(q)$.

For $X=A_{r}(q)$ with $r \geq 4$, we first use the same argument with an $A_{r-1^{-}}$ parabolic: if $\delta=a_{1} \ldots a_{r}$, we see that $a_{1} \ldots a_{r-1}$ is equal to $0 \ldots 0,10 \ldots 0$ or $0 \ldots 01$ (or, if $r=4$, to 010 ). As $V \downarrow X$ is self-dual, we conclude that $a_{1} \ldots a_{r}=10 \ldots 01$. Now choose another parabolic $Q_{X}^{(1)} L_{X}^{(1)}$ of type $A_{2} \times A_{r-3}$; then $C_{V}\left(Q_{X}^{(1)}\right)$ has dimension $3(r-2)$, and we deduce using 7.2 that $3(r-2) \leq 8$. Hence $r=4$ and $V \downarrow X=V_{X}(1001)$; but $\operatorname{dim} V_{X}(1001) \leq 24$, which is a contradiction.

Next let $X=B_{3}(q)$, with $\delta=a b c$. From an $A_{2}$-parabolic and 7.8 we get $a b=00,10,01,20,02,11$ or 22 ; and from a $B_{2}$-parabolic we get $b c=00,10,01,20,02$ or 11 . Hence $\delta$ is in the following list:

$$
\text { 001, 002, 100, 101, 102, 010, 011, 200, 201, 202, 020, 110,111, } 220 .
$$

If $\delta=001,002,100,101,010$ or 200 , then $V_{X}(\delta)$ appears in a tensor product $V_{X}\left(\lambda_{i}\right) \otimes$ $V_{X}\left(\lambda_{j}\right)$ with $i, j \in\{1,3\}$, hence has dimension less than 77 ; and if $\delta=201$ then $p \neq 2$ and the acting group is the simply connected group $\operatorname{Spin}_{7}(q)$, with centre of order 2, contrary to the fact that $Z(X)=1$. All other possibilities for $\delta$ are in Table 6 .

Now consider $X=B_{r}(q)$ with $r \geq 4$ and $\delta=a_{1} \ldots a_{r}$. Observe that $r \leq 8$, as can be seen from an easy inductive argument (embed a $B_{r-1}$-parabolic of $X$ in a parabolic of $G$, and repeat). Working with parabolics of types $A_{r-1}$ and $B_{r-1}$ as above, we find $a_{1} \ldots a_{r-1}=00 \ldots 0,10 \ldots 0$ or $0 \ldots 01$ (or 010 with $r=4$ ), and $a_{2} \ldots a_{r}=00 \ldots 0$ (or 100,200,001 with $r=4$, or 0001 with $r=5$ ). Hence $\delta$ is one of the following:

$$
10 \ldots 0,0001,00001,1001,10001,0100 \text {. }
$$

The first three cases have dimension $2 r+1$ or $2 r, 16$ and 32 respectively, so are impossible; and the last has dimension less than 77 . If $\delta=10001$, then an $A_{2} B_{2^{-}}$ parabolic acts on the fixed space of its radical as $10 \otimes 01$, so this fixed space has dimension 12 , contrary to 7.2. This leaves only $\delta=1001$; here also $p=2$, since otherwise the acting group has centre of order 2 again. But when $p=2$, $V_{B_{4}}(1001) \cong V_{B_{4}}(1000) \otimes V_{B_{4}}(0001)$ (see [Bor2, 7.6]), of dimension 8.16, so this case cannot occur either.

The cases $X=C_{r}(q)(r \geq 3)$ or $D_{r}(q)(r \geq 4)$ are handled in just the same way as $B_{r}(q)$ (for $D_{r}(q)$ we use two $A_{r-1}$-parabolics).

For $X=F_{4}(q)$ with $\delta=a b c d$, use of a $B_{3}$-parabolic and 7.2 gives $a b c=$ $000,100,200$ or 001 , and use of a $C_{3}$-parabolic gives $b c d=000,100,010$ or $001(p=$ $2)$. Hence $\delta=1000,1001(p=2), 2000,0010$ or $0001(p=2)$. Of these, the first and last have dimension less than 77 , and $1001 \cong 1000 \otimes 0001$ ([Bor2, 7.6]), of dimension 26.26; the rest are in Table 6 .

When $X=E_{6}(q), E_{7}(q)$ or $E_{8}(q)$, use of suitable parabolics forces $\delta=\lambda_{2}, \lambda_{1}$ or $\lambda_{8}$ respectively, whence $X$ has the same type as $G$, contrary to our original assumption.

This completes the case where $X$ is of untwisted type. 
Now suppose $X={ }^{2} A_{3}(q)$ and $\delta=a b a$. Choose a parabolic $P_{X}=Q_{X} L_{X}$ of $X$ with $L_{X}^{\prime} \cong A_{1}\left(q^{2}\right)$. Then $C_{V}\left(Q_{X}\right) \downarrow L_{X}=a \otimes a^{(q)}$. Also $Q_{X}$ is non-abelian, so we deduce from 7.2 that $\operatorname{dim} C_{V}\left(Q_{X}\right)=(a+1)^{2} \leq 8$, whence $a \leq 1$. The usual argument using an $A_{1}(q)$-parabolic shows that $b \leq 7$. Now choose a subgroup $B \cong B_{2}(q)$ of $X$. Then $V \downarrow B$ has a composition factor $V_{B}(b, 2 a)$. Using the list of $B_{2}$-modules given in the proof of 7.6 , we see that either $a=0, b \leq 7$ or $a=1, b \leq 5$, as in Table 6.

Now consider $X={ }^{2} A_{4}(q), \delta=a b b a$. There is a parabolic $Q_{X} L_{X}$ with $L_{X} \cong$ ${ }^{2} A_{2}(q)$ and $C_{V}\left(Q_{X}\right) \downarrow L_{X} \cong V_{L_{X}}(b b)$. Hence by 7.8 , we have $b \leq 2$; moreover, if $b=2$ then $\operatorname{dim} C_{V}\left(Q_{X}\right)=27$; but this is impossible by 7.2 , as $Q_{X}$ is nonabelian. Thus $b \leq 1$. As in the previous paragraph, we see that $a \leq 1$. Clearly 0000 and 1001 have dimension less than 77, leaving 0110 and 1111, as in Table 6 . For $X={ }^{2} A_{r}(q)$ with $r \geq 5$ and $\delta=a_{1} \ldots a_{r}$, an $A_{2}\left(q^{2}\right)$ parabolic $Q_{X} L_{X}$ has $C_{V}\left(Q_{X}\right) \downarrow L_{X}=a_{1} a_{2} \otimes a_{2} a_{1}^{(q)}$, whence $a_{1}=a_{2}=0$, and a ${ }^{2} A_{r-2}(q)$ parabolic now gives either $a_{i}=0$ for all $i$, or $r=5, \delta=00100$; in the latter case, $V_{X}(\delta)$ has dimension 20, a contradiction.

Next let $X={ }^{2} D_{r}(q)(r \geq 4)$. Note again that $r \leq 9$. Use of 7.8 with parabolics of types ${ }^{2} D_{r-1}$ and $A_{r-2}$ yield the following possibilities for $\delta$ :

$$
\lambda_{1}, \lambda_{r-1}, \lambda_{r}, \lambda_{1}+\lambda_{r-1}, \lambda_{1}+\lambda_{r}, 01000,2000,2010,2001,0100,1100 .
$$

The first three possibilities have dimensions $2 r, 2^{r-1}, 2^{r-1}$ respectively, so these cannot hold. For the next two cases, when $r \geq 6$ an $A_{r-4}(q) \times{ }^{2} A_{3}(q)$ parabolic rules them out; for $r=5$ they are not self dual; and for $r=4$ they have dimension less than 77. Finally, the cases with $\delta=01000,2000,0100$ have dimension less than 77 also. This leaves just the possibilities in Table 6 .

Similarly, for $X={ }^{2} E_{6}(q)$ we use parabolics of types ${ }^{2} A_{5}$ and ${ }^{2} D_{4}$ to force $\delta=\lambda_{1}, \lambda_{6}, \lambda_{2}, \lambda_{1}+\lambda_{2}$ or $\lambda_{6}+\lambda_{2}$. Of these, only $\lambda_{2}$ is self dual. But this is the high weight of the adjoint module for $X$, which means that $X$ and $G$ must have the same type, contrary to assumption.

It remains to deal with $X={ }^{2} F_{4}(q)$ or ${ }^{3} D_{4}(q)$. In the first case, $V \downarrow X=$ $V_{X}(a b c d) \cong V_{X}(a b 00) \otimes V_{X}(00 c d)$. Each tensor factor is either trivial or has dimension at least 26 , so one of them, say $00 \mathrm{~cd}$, is trivial. Thus $V \downarrow X=1000,0100$ or 1100; the first has dimension 26, and the others are in Table 6.

Finally, let $X={ }^{3} D_{4}(q)$, and suppose $\delta=a b c d$. Pick a parabolic $Q_{X} L_{X}$ with $L_{X}^{\prime} \cong A_{1}\left(q^{3}\right)$. Then $C_{V}\left(Q_{X}\right) \downarrow L_{X}=a \otimes c^{(q)} \otimes d^{\left(q^{2}\right)}$. Moreover $Q_{X}$ is non-abelian, so we deduce from 7.2 and 1.1 that $(a+1)(c+1)(d+1) \leq 8$. Therefore, taking $a \geq c \geq d$, we have

$$
(a, c, d)=(a, 0,0)(a \leq 7),(a, 1,0)(a \leq 3) \text { or }(1,1,1) .
$$

Now take a subgroup $C=G_{2}(q)$ of $X$, so that $V \downarrow C$ has a composition factor $V_{C}(a+c+d, b)$. This module is restricted unless $a c d=210,110$ or 111 and $p \leq 3$. These cases are included in Table 6 of the statement. In the restricted case, we see from the list of $G_{2}$-modules given in the proof of 7.6 that

$$
a+c+d, b=40,30,21,20,13,12,11,10,03,02 \text {, or } 01 .
$$

Hence $a b c d$ is as in Table 6 .

The proof of Theorem 4 is completed by the next lemma. 
Lemma 7.10. Let $X$ and $\delta$ be as in Table 6 . Then $\operatorname{dim} V_{X}(\delta)$ is not equal to $77-\delta_{p, 3}, 133-\delta_{p, 2}$ or 248 .

Proof. For many of the possibilities in Table 6 , the dimension of $V_{X}(\delta)$ is given by $[\mathrm{BW}]$ or [GS]. For several of the remaining cases, counting conjugates of subdominant weights in $V_{X}(\delta)$ shows that $\operatorname{dim} V_{X}(\delta)>248$. The final surviving cases are handled using the programme described in [GS] to determine the precise dimension of $V_{X}(\delta)$.

This completes the proof of Theorem 4 .

\section{Proof of Theorem 6 And Corollaries}

We begin with the proof of Theorem 6 . As in the hypothesis of Theorem 6 , let $G$ be a simple adjoint algebraic group of exceptional type in characteristic $p$, and $\sigma$ be a Frobenius morphism such that $L=O^{p^{\prime}}\left(G_{\sigma}\right)$ is a finite simple group of exceptional Lie type. Let $L_{1}$ be a finite group with socle $L$ (i.e. $L \leq L_{1} \leq$ Aut $L$ ). Suppose that $H$ is a maximal subgroup of $L_{1}$ such that $F^{*}(H)=X=X(q)$, a simple group of Lie type over $\mathbb{F}_{q}$, with $q$ as in the hypothesis of Theorem 1 . Assume further that $X$ is not of the same type as $G$ (otherwise (i) of Theorem 6 holds).

By Corollary 5 , there is a proper connected subgroup $\bar{X}$ of $G$ containing $X$ such that $X$ and $\bar{X}$ fix the same subspaces of $L(G)$.

Let $\mathcal{M}$ be the set of all $X$-invariant subspaces of all $G$-composition factors of $L(G)$, and define

$$
Y=\bigcap_{W \in \mathcal{M}} G_{W}
$$

As every automorphism of $L$ extends to a morphism $G \rightarrow G$, it follows from 1.12(iii) that $Y$ is $H\langle\sigma\rangle$-stable. Moreover, by Theorem 4, $Y$ is a proper closed subgroup of $G$, and $X<\bar{X} \leq Y$. Let $Z$ be a maximal connected $H\langle\sigma\rangle$-invariant proper subgroup of $G$ containing $Y^{0}$. By the maximality of $H$ we have $H=N_{L_{1}}(Z)$. Clearly $Z$ is reductive (otherwise $H$ normalizes $R_{u}(Z)_{\sigma}$, hence is parabolic). Hence $X=O^{p^{\prime}}\left(Z_{\sigma}\right)$. Consequently (ii) of Theorem 6 holds.

This completes the proof of Theorem 6 .

Remark. As remarked after Theorem 6 in the Introduction, the above proof shows, more generally, that if $H$ is any (not necessarily maximal) subgroup of $L_{1}$ such that $F^{*}(H)=X(q)$ with $q$ as in Theorem 1 , then $H \leq N\left(\bar{X}_{\sigma}\right)$ for some $\bar{X}$ as in conclusion (ii) of Theorem 6.

Proof of Corollary 7. Assume the hypotheses of Corollary 7, so that $H$ is a maximal subgroup of $L_{1}$ as above, and $F^{*}(H)=X=X(q)$ with $q$ as in Theorem 1 . We may take it that $K=\overline{\mathbb{F}}_{p}$, the algebraic closure of $\mathbb{F}_{p}$, since the conclusions of Corollary 7 concern finite groups only. (This allows us to apply 1.13 at a suitable point in the argument.)

Assume that $H$ is not a subgroup of maximal rank, and that $X(q)$ is not of the same type as $G$. By Theorem 6 , we have $X=O^{p^{\prime}}\left(\bar{X}_{\sigma}\right)$, where $\bar{X}$ is a maximal closed connected reductive $H\langle\sigma\rangle$-stable subgroup of $G$. By assumption, $\bar{X}$ does not contain a maximal torus of $G$. Moreover, $Z(\bar{X})^{0}=1$, since otherwise $H \leq N\left(C\left(Z(\bar{X})^{0}\right)\right.$, a group of maximal rank, which is impossible as $H$ is not of maximal rank by assumption. Therefore $\bar{X}$ is semisimple. If $\bar{X}$ is not simple, then, by [LS1, Theorem 
1], $\bar{X}$ is as in the second column of [LS1, Table II]; from this we see that $O^{p^{\prime}}\left(\bar{X}_{\sigma}\right)$ is not simple, a contradiction.

Hence $\bar{X}$ is simple. Therefore, since $X=X(q)=O^{p^{\prime}}\left(\bar{X}_{\sigma}\right)$, by 1.13 we have $q=q_{1}$ (where $G_{\sigma}=G\left(q_{1}\right)$ ), as in (iii) of Corollary 7. Moreover, if $p>N(X, G)$, then the possibilities for $\bar{X}$ are given by [LS1, Theorem 1]; note also from [LS1, Theorem 1] that these maximal $H\langle\sigma\rangle$-stable subgroups $\bar{X}$ are also maximal $\sigma$-stable.

There remain two points to establish: first, that the fixed point groups $X(q)=$ $O^{p^{\prime}}\left(\bar{X}_{\sigma}\right)$ are as in conclusion (iii) of Corollary 7; and second, the conjugacy statement at the end of Corollary 7.

The conjugacy statement is straightforward: first, the assertion of the uniqueness of Aut $(G)$-classes of maximal connected subgroups $\bar{X}$ is given by [Se2, Theorem 1]. And second, by [SS, I,2.7], the $G_{\sigma}$-classes contained in a given $G$-class of subgroups $\bar{X}$ correspond to classes in the group $H^{1}\left(\sigma, N_{G}(\bar{X}) / \bar{X}\right)$; however, clearly $N_{G}(\bar{X}) / \bar{X}$ has order 1 unless $\bar{X}=A_{2}$, in which case it has order 2 by Lemma 8.1 below, so the conclusion is immediate.

The statement that the fixed point groups $X(q)=O^{p^{\prime}}\left(\bar{X}_{\sigma}\right)$ are as in conclusion (iii) of Corollary 7 is clear, except when $\bar{X}=A_{2}$, in which case we must establish that both the fixed point groups $A_{2}(q)$ and ${ }^{2} A_{2}(q)$ arise. This follows from the next lemma.

Lemma 8.1. Let $\bar{X} \cong A_{2}$ be a maximal connected subgroup of $G=E_{6}$ or $E_{7}$. Then $N_{G}(\bar{X})$ induces a nontrivial graph automorphism on $\bar{X}$ (and hence $N_{G}(\bar{X}) / \bar{X}$ has order 2$)$.

Proof. We may assume that $G$ is of adjoint type. When $G=E_{6}$, the conclusion is immediate from [Te2, Claim on p. 314]. So assume from now on that $G=E_{7}$. The class of maximal subgroups $A_{2}$ in $E_{7}$ for $p \geq 7$ is constructed in [Se2, 5.8].

Choose a parabolic subgroup $Q J_{\alpha} T_{\alpha}$ of $\bar{X}$, where $J_{\alpha}$ is a fundamental $S L_{2}, T_{\alpha}$ a rank 1 torus and $Q$ the unipotent radical. Then by [Se2, p. 83], $C_{G}\left(T_{\alpha}\right)=T_{2} A_{1} A_{4}$, $J_{\alpha}$ projects onto the factor $A_{1}$, and $J_{\alpha}$ projects onto a regular $A_{1}$ in the factor $A_{4}$.

We claim that there is an involution $z \in N_{G}\left(T_{2} A_{1} A_{4}\right)$ normalizing $J_{\alpha}$ and inverting $T_{2}$. To see this, we work first in $E_{8}$, and observe that there is an involution $u$ normalizing a maximal rank subgroup $A_{4} A_{4}$ of $E_{8}$, inducing a graph automorphism on each factor. Then $u$ normalizes a fundamental $A_{1}$ in one of the factors $A_{4}$, inducing a nontrivial inner automorphism on this $A_{1}$. Therefore $u \in A_{1} E_{7}$ and is a product $y z$ of elements $y, z$ of order 4 , where $y \in A_{1}, z \in E_{7}$, and $y^{2}=z^{2}$ is a generator of $Z\left(E_{7}\right)$. Then $z$ projects to an involution in the adjoint group of type $E_{7}$. Now $C_{A_{4} A_{4}}\left(A_{1}\right)=T_{1} A_{2} A_{4}$; therefore $u$, hence $z$, normalizes this group, acting as a graph automorphism on the $A_{2}, A_{4}$ factors and inverting the $T_{1}$. Now $z$ centralizes a subgroup $B_{2}$ of the $A_{4}$ factor, and a subgroup $P S L_{2}$ therein. Adjusting $z$ by a suitable involution of this $P S L_{2}$, we obtain an element $z$ normalizing a subgroup $T_{2} A_{1} A_{4}$ of $T_{1} A_{2} A_{4}$, acting in the fashion claimed at the beginning of this paragraph.

Let $t$ be the central involution in $J_{\alpha}$. Then $C_{G}(t)=A_{1} D_{6}$, and $L(G) \downarrow A_{1} D_{6}=$ $L\left(A_{1} D_{6}\right) \oplus\left(V_{A_{1}}(1) \otimes V_{D_{6}}\left(\lambda_{5}\right)\right)$. The restriction of $V_{D_{6}}\left(\lambda_{5}\right)$ to the projection of $J_{\alpha}$ in $A_{4}$ (which lies in $D_{6}$ ) is $6^{2} / 4^{2} / 2^{2} / 0^{2}$; tensoring with $V_{J_{\alpha}}(1)$, we obtain

$$
V_{A_{1}}(1) \otimes V_{D_{6}}\left(\lambda_{5}\right) \downarrow J_{\alpha}=7^{2} / 5^{4} / 3^{4} / 1^{4} .
$$

We claim that there is an 8 -dimensional $J_{\alpha}$-submodule isomorphic to $1^{4}$. Two of the required four summands are provided by $L(Q)$ and $L\left(Q^{-}\right)$. Now consider 
the action of $T=T_{\alpha}$; say it acts with weight $\beta$ on $L(Q)$, hence with weight $-\beta$ on $L\left(Q^{-}\right)$. Now $T_{2}$ centralizes $J_{\alpha}$, so acts on the homogeneous component $A$ of the socle of $L(G) \downarrow J_{\alpha}$ corresponding to high weight 1 . It also centralizes $T$, hence acts on the $\beta$ and $-\beta$ weight spaces in $A$; and $z$ interchanges these. If the $\beta$ weight space is just 2 -dimensional (i.e. equal to $L(Q)$ ), then so is the $-\beta$ weight space, and conversely. But then the Lie algebra span of this part of the socle is just $\left\langle L(Q), L\left(Q^{-}\right)\right\rangle=L(\bar{X})$. However, this is not $T_{2}$-invariant, so this is a contradiction. Therefore the $\beta$ and $-\beta$ weight spaces of $A$ have dimension 4 , and $A \cong 1^{4}$, proving the claim.

Let $W_{\beta}, W_{-\beta}$ be the $\pm \beta$ weight spaces in $A$. These are fixed by $T_{2}$ and interchanged by $z$. If $C_{T_{2}}\left(W_{\beta}\right)^{0} \neq 1$, then there is a 1 -dimensional torus acting trivially on $W_{\beta}$, hence also on $W_{-\beta}$ (as $z$ inverts $T_{2}$ ). But this torus then acts trivially on $\left\langle L(Q), L\left(Q^{-}\right)\right\rangle=L(\bar{X})$ (Lie algebra span), which is impossible (as $\bar{X}$ is maximal). Therefore $C_{T_{2}}\left(W_{\beta}\right)^{0}=1$. Consequently $W_{\beta}=W_{1} \oplus W_{2}$, a sum of two 2-dimensional $T_{2}$-weight spaces with different $T_{2}$-kernels; similarly $W_{-\beta}=W_{3} \oplus W_{4}$.

Now none of the $W_{i}$ is equal to $L(Q)$ or $L\left(Q^{-}\right)$(otherwise we would get a 1dimensional torus acting trivially on $L(\bar{X})$ as above). Hence $L(Q)$ is diagonal in $W_{1} \oplus W_{2}$. As a $J_{\alpha} T_{2}$-module, we have $W_{\beta} \cong M \otimes N$, where $M$ is a natural 2dimensional module for $J_{\alpha}$ and $N$ is a direct sum of two 1-dimensional $T_{2}$-modules with $T_{2}$-kernels having different connected components. As $T_{2}$ has three orbits on the nonzero vectors of $N$, it follows that $T_{2}$ has three orbits on the set of 2dimensional $J_{\alpha}$-invariant subspaces of $W_{\beta}$. Since $L\left(Q^{-}\right)^{z}$ is such a subspace, and is diagonal in $W_{1} \oplus W_{2}$, there is therefore an element $a \in T_{2}$ such that $L\left(Q^{-}\right)^{z a}=$ $L(Q)$. Then $z a$ is an involution normalizing the Lie algebra span $\left\langle L(Q), L\left(Q^{-}\right)\right\rangle=$ $L(\bar{X})$. Consequently $z a$ normalizes $\bar{X}$, inducing a graph automorphism, and this completes the proof of the lemma.

Proof of Corollary 8. Let $L_{1}$ be as in the first paragraph of this section, and let $H$ be a maximal subgroup of $L_{1}$. Assume first that $H$ is not almost simple. Then by [LS1, Theorem 2], one of the following holds:

(i) $H=N\left(\bar{X}_{\sigma}\right)$, where $\bar{X}$ is a maximal connected $H\langle\sigma\rangle$-stable subgroup of $G$;

(ii) $H$ is the normalizer of a subgroup of the same type as $G$;

(iii) $H$ one of the local subgroups $2^{3} \cdot L_{3}(2), 3^{3} \cdot L_{3}(3), 3^{3+3} \cdot L_{3}(3), 2^{5+10} \cdot L_{5}(2)$, $5^{3} . L_{3}(5)$ (in $G_{2}, F_{4}, E_{6}, E_{8}, E_{8}$ respectively), given in [LSS, Theorem 1(II)];

(iv) $F^{*}(H)=\operatorname{Alt}_{5} \times \operatorname{Alt}_{6}$.

Taking $c>2^{15}\left|L_{5}(2)\right|$, we see that (i) or (ii) of Corollary 8 holds for $|H|>c$.

Now assume that $H$ is almost simple, and let $X=F^{*}(H)$. If $X$ is alternating, sporadic, or of Lie type in $p^{\prime}$-characteristic, then $H$ has bounded order (see [LSS, $\S 4]$ for instance); so we may choose $c$ so that this does not occur for $|H|>c$. Finally, let $X=X(q)$ with $q=p^{e}$. The $B N$-rank of $X$ is at most that of $G$ (since a parabolic of $X$ must lie in a parabolic of $G$ ), so again choosing $c$ appropriately, we may assume that $q$ satisfies the hypothesis of Theorem 1. Now the conclusion follows from Theorem 6 .

\section{Proofs of Theorems 9 And 10}

For the proof of Theorem 9, we shall need the information on first cohomology groups given in the next two results. The first is taken from [Ja, 2.3].

Proposition 9.1 (Jantzen). Let $Y$ be a simply connected simple algebraic group over $\overline{\mathbb{F}}_{p}$, and let $\sigma$ be a Frobenius morphism of $Y$ such that $Y_{\sigma}$ is not of type ${ }^{2} B_{2}$, 
${ }^{2} G_{2}$ or ${ }^{2} F_{4}$. Let $Y_{\sigma}=Y(q)$, a finite group of Lie type over $\mathbb{F}_{q}$, with $q=p^{r}$, and let $\alpha_{0}$ be the highest short root of the root system of $Y$. Suppose that $\lambda$ is a dominant weight for $Y$ satisfying

$$
\left\langle\lambda, \alpha_{0}^{\mathrm{v}}\right\rangle \leq \begin{cases}p^{r}-2 p^{r-1}-3, & \text { if } Y \neq G_{2}, \\ p^{r}-3 p^{r-1}-3, & \text { if } Y=G_{2},\end{cases}
$$

and let $V=V_{Y}(\lambda)$. Then the natural restriction map

$$
H^{1}(Y, V) \rightarrow H^{1}\left(Y_{\sigma}, V\right)
$$

is an isomorphism.

Proposition 9.2. Let $Y$ be a simple algebraic group in characteristic $p$, let $Y(q)=$ $Y_{\sigma}$ be a quasisimple group of Lie type over $\mathbb{F}_{q}, q=p^{e}$, and let $V=V_{Y(q)}(\lambda)$. Then $H^{1}(Y(q), V)=0$ for all $\lambda$ in Table $\%$.

\section{TABLE 7}

\begin{tabular}{lll}
$Y(q)$ & $\lambda$ & conditions on $p, q$ \\
\hline$A_{r}^{\epsilon}(q)$ & $\lambda_{1}, \lambda_{2}, \lambda_{3}$ & $q>3$, and $p>2$ if $r=1$ \\
$B_{r}(q)(r \geq 2)$ & $\lambda_{1}, \lambda_{r}$ & $p>2$ if $\lambda=\lambda_{1}$ or $r=2$, \\
& & $Y(q) \neq B_{2}(3)$ \\
$D_{r}^{\epsilon}(q)(r \geq 4)$ & $\lambda_{1}, \lambda_{r-1}, \lambda_{r}$ & $p>2, q>3$ \\
$G_{2}^{\epsilon}(q)$ & 10 & $p>2$ \\
$C_{4}(q)$ & $\lambda_{1}, \lambda_{3}$ & $p \neq 3, q>8$ \\
$F_{4}^{\epsilon}(q)$ & $\lambda_{4}$ & \\
$E_{6}^{\epsilon}(q)$ & $\lambda_{1}$ & \\
$E_{7}(q)$ & $\lambda_{7}$ & \\
$A_{2}^{\epsilon}(q)$ & $20,30,60,11,21,22$, & \\
& $10 \otimes 10^{\left(p^{i}\right)}, 10 \otimes 01^{\left(p^{i}\right)}$, & \\
& $10 \otimes 11^{\left(p^{i}\right)}, 10 \otimes 20^{\left(p^{i}\right)}$, & \\
& $10 \otimes 02^{\left(p^{i}\right)}$ & $p \geq 5, q \geq 11(p \geq 7$ for 20, \\
$B_{2}(q)$ & $02,03,20,11,13$, & $p=3$ allowed for $\lambda=02)$ \\
& $10 \otimes 10^{\left(p^{i}\right)}, 10 \otimes 01^{\left(p^{i}\right)}$, & \\
& $01 \otimes 01^{\left(p^{i}\right)}, 01 \otimes 02^{\left(p^{i}\right)}$ & \\
$G_{2}(q)$ & $20,01,11,10 \otimes 10^{\left(p^{i}\right)}$ & $p \geq 11$ \\
$A_{3}^{\epsilon}(q)$ & $101,110,200,100 \otimes 100^{\left(p^{i}\right)}$, & $p>2, q \geq 11$ \\
& $100 \otimes 001^{\left(p^{i}\right)}, 100 \otimes 010^{\left(p^{i}\right)}$ & \\
$B_{3}(q)$ & $010,002,101,001 \otimes 001^{\left(p^{i}\right)}$ & $p>2, q \geq 11$ \\
$C_{3}(q)$ & $100,010,001,110$ & $p>2(p>3$ for $\lambda=010), q \geq 11$ \\
$C_{4}(q)$ & 0100,0010 & $p>2, q \geq 11$ \\
$D_{4}^{\epsilon}(q)$ & 0100,0011 & $p>2, q \geq 11$ \\
\hline
\end{tabular}

Proof. For $Y(q)$ as in the first eight rows of the table (i.e. $Y(q)=A_{r}^{\epsilon}(q), \ldots$, $E_{7}(q)$ ), the result follows immediately from [JP] and [LS3, 1.8]. For the other cases, our assumptions on $p, q$ imply that $H^{1}(Y(q), V) \cong H^{1}(Y, V)$ by 9.1. (For cases where $\lambda$ is non-restricted, we twist $V$ by an automorphism of $Y(q)$ to take $i \leq r / 2$.) It follows from [LS2, 1.7 and 1.9-1.15] that $H^{1}(Y, V)=0$ in all cases. 
We shall also need some elementary lower bounds for the numbers $t(G)$ defined in the Introduction.

Proposition 9.3. We have $t\left(G_{2}\right) \geq 12$, and $t(\Sigma(G)) \geq 16$ for $G=F_{4}, E_{6}, E_{7}, E_{8}$.

Proof. For $G=G_{2}$, take simple roots $\alpha$ (short) and $\beta$ (long), and set

$$
y=(3 \alpha+2 \beta)-(-3 \alpha-\beta)=6 \alpha+3 \beta, \quad z=\beta-(-\beta)=2 \beta
$$

(the middle terms in these equations are written simply to demonstrate that $y$ and $z$ are root differences); then $12 \alpha=2 y-3 z$, but $6 \alpha$ and $4 \alpha$ are not in $\mathbb{Z} y+\mathbb{Z} z$. Hence $t(\Sigma(G)) \geq 12$.

For $G=F_{4}$, take simple roots $\alpha, \beta, \gamma, \delta$ with $\alpha$ and $\beta$ long and $\beta$ joined to $\gamma$. Set $x=(\alpha+2 \beta+2 \gamma)-(-\alpha-\beta-2 \gamma)=2 \alpha+3 \beta+4 \gamma, y=(\alpha+\beta)-(-\beta)=\alpha+2 \beta$, $z=\alpha-(-\alpha)=2 \alpha$; then $16 \gamma=4 x-6 y-z$, but $8 \gamma$ is not in $\mathbb{Z} x+\mathbb{Z} y+\mathbb{Z} z$. Hence $t(\Sigma(G)) \geq 16$.

For $G=E_{6}, E_{7}$ or $E_{8}$ take four simple roots $\alpha, \beta, \gamma, \delta$ forming an $A_{4}$ subsystem. Set $w=(\alpha+\beta)-(-\alpha)=2 \alpha+\beta, x=(\beta+\gamma)-(-\beta)=2 \beta+\gamma, y=(\gamma+\delta)-(-\gamma)=$ $2 \gamma+\delta, z=\delta-(-\delta)=2 \delta$; then $16 \alpha=8 w-4 x+2 y-z$, but $8 \alpha$ is not in $\mathbb{Z} w+\mathbb{Z} x+\mathbb{Z} y+\mathbb{Z} z$. Hence $t(\Sigma(G)) \geq 16$.

Proof of Theorem 9. Let $G$ be a simple exceptional adjoint algebraic group over the algebraically closed field $K$ of characteristic $p$, let $X=X(q)<G$ with $q$ as in the hypotheses of Theorem 1, and assume that $p>N^{\prime}(X, G)$ (as defined before the statement of Theorem 9). Suppose that $X<P=Q L$, a parabolic subgroup of $G$ with unipotent radical $Q$ and Levi subgroup $L$. We aim to show that $X$ lies in a $Q$-conjugate of $L$.

We follow closely the proof of [LS2, Theorem 1], which appears in [LS2, §3]. Assume first that $P$ is minimal subject to containing $X$, and choose a subgroup $X_{1}$ of $L$ such that $Q X=Q X_{1}$.

Suppose that $X \approx A_{1}(q)$. By Theorem $1, X_{1}<\bar{X} \leq L$, where $\bar{X}$ is connected and $X_{1}$ and $\bar{X}$ stabilize the same subspaces of the Lie algebra of $L$. Since $\bar{X}$ lies in no parabolic subgroup of $L$, it is semisimple, say $\bar{X}=\bar{X}_{1} \ldots \bar{X}_{r}$ with all $\bar{X}_{i}$ simple. The fact that $X_{1}$ is irreducible on each $\bar{X}$-composition factor of $L\left(\bar{X}_{i}\right)$ implies that all the $\bar{X}_{i}$ are of the same type as $X$. Therefore $X$ lies in a connected simple subgroup $\bar{X}_{0}$ of $L$ of the same type (a diagonal subgroup of $\bar{X}_{1} \ldots \bar{X}_{r}$ ). Hence the possible embeddings of $X_{1}$ in $L$ are given by [LS2, 3.3] (with the same proof). The $L^{\prime}$-composition factors within $Q$ have the structure of rational $K L^{\prime}$-modules, with high weights given by [LS2, 3.1]. We deduce as in the proof of [LS2, 3.4] that the restrictions to $X_{1}$ of such modules have composition factors with high weights $\lambda$ all twists of those occurring in the table of Proposition 9.2. Moreover, the conditions on $p, q$ given in the table are satisfied (since we are assuming $p>N^{\prime}(X, G)$ and $q>9)$, except in the following cases:

(1) $X_{1} \cong B_{r}(q)(r \geq 5), p=2, \lambda=\lambda_{1}, L^{\prime}=D_{r+1}$

(2) $X_{1} \cong C_{3}(q), p=3, \lambda=\lambda_{2}, L^{\prime}=A_{5}$

(3) $X_{1} \cong F_{4}(q), p=3, \lambda=\lambda_{4}, L^{\prime}=E_{6}$.

Therefore, excluding these cases, the group $Q X_{1}$ has just one $Q$-conjugacy class of complements to $Q$, and so $X$ is equal to a $Q$-conjugate of $X_{1}$, as required. Finally, cases $(1),(2),(3)$ above are dealt with exactly as in the proof of [LS2, 3.6], using 9.1.

Now let $X \cong A_{1}(q)$. First assume that $q=p$. By hypothesis, we have $q>$ $2 t(\Sigma(G))$; and by $9.3, t(\Sigma(G)) \geq 12$ if $G=G_{2}, t(\Sigma(G)) \geq 16$ otherwise; so $q=p>$ 24 if $G=G_{2}$, and $q=p>32$ otherwise. By 1.2 , we have $H^{1}\left(X, V_{X}(r)\right)=0$ when 
$r<p-3$. Now [LS2, 2.4],together with the first row of the table of [LS2, 2.13] shows that all $X$-composition factors within $Q$ have high weights at most 27 (at most 4 if $\left.G=G_{2}\right)$. Hence $H^{1}(X, V)=0$ for all such composition factors $V$, and we deduce as before that $X$ is a $Q$-conjugate of $X_{1}$.

Finally, suppose $q=p^{e}$ with $e \geq 2$. By 1.2 , if $H^{1}\left(X, V_{X}(r)\right) \neq 0$ then $V_{X}(r)$ must be a twist of the module $V_{X}(p-2) \otimes V_{X}(1)^{(p)}$. Now the proof of [LS2, 3.5] shows that such a module can appear as an $X$-composition factor within $Q$ only if

$$
p=11 \text { or } 17, L^{\prime}=D_{7}\left(\text { and } G=E_{8}\right) .
$$

The embedding of $X Q / Q$ in $D_{7}$ is such that the action on the natural module is $10 \oplus 2^{(p)}$, and the composition factor $(p-2) \otimes 1^{(p)}$ occurs within the module $Q / Q^{\prime}$ with multiplicity 1 ; moreover, no nontrivial twist of this composition factor occurs within $Q / Q^{\prime}$.

Suppose $X$ does not lie in a $Q$-conjugate of $L$. By 1.3, the restriction map $H^{1}\left(A_{1}(K),(p-2) \otimes 1^{(p)}\right) \rightarrow H^{1}\left(X,(p-2) \otimes 1^{(p)}\right)$ is injective. Hence there is a connected subgroup $F$ of $Q L$ containing $X Q^{\prime}$ such that $F / Q^{\prime}$ is of type $A_{1}$. A similar analysis took place in [LS2, 3.7], except that there we were dealing with a connected subgroup of type $A_{1}$ instead of the finite group $X$. In [LS2, 3.7] it is shown that this connected $A_{1}$ subgroup lies in a $Q$-conjugate of $L$. This implies that the group $F$ does not contain a closed complement to $Q^{\prime}$. Now $Q^{\prime} \downarrow X=10 \oplus 2^{(p)}$. Since $F$ contains $X$, it follows that the restriction map $H^{2}\left(A_{1}(K), 10 \oplus 2^{(p)}\right) \rightarrow$ $H^{2}\left(X, 10 \oplus 2^{(p)}\right)$ is not injective. However, this map is in fact injective: to see this, we verify the injectivity condition (5.4) of [CPSK] for $n=2$, and the isomorphism condition (5.5) for $n=1$, upon which the assertion follows from [CPSK, 5.1].

Therefore $X$ lies in a $Q$-conjugate of $L$.

We have now proved Theorem 9 (except for the last sentence concerning $\sigma$ stability), in the case where $P=Q L$ is minimal subject to containing $X$. The proof for non-minimal parabolics containing $X$ follows as in [LS2, p. 43].

We finally deal with the $\sigma$-stability statement in the last sentence of Theorem 9 . Suppose then that $X<G_{\sigma}$ and $X<P=Q L$, a $\sigma$-stable parabolic. By what we have already proved, we can assume that $X<L$. Define

$$
\Delta=\left\{L^{u}: u \in Q, X<L^{u}\right\} .
$$

Observe first that if $X<L^{u}(u \in Q)$, then $X^{u^{-1}}<L \cap Q X=X$, whence $u \in$ $N_{Q}(X)=C_{Q}(X)$. Consequently $C_{Q}(X)$ acts transitively on $\Delta$. Write $C=C_{Q}(X)$.

We claim now that $C$ is connected. To see this, let $T=Z(L)^{0}$. Then $X<C_{G}(T)$, so $T$ normalizes $C$. Obviously $T$ acts trivially on the finite group $C / C^{0}$, so if this group is nontrivial, then $T$ fixes a nonzero vector in some composition factor of $Q \downarrow T$. However $C_{Q}(T)=1$, so this is impossible. Hence $C$ is connected, as claimed.

Thus the connected group $C$ acts transitively on the $\sigma$-stable set $\Delta$. It now follows from [SS, I,2.7] that $\Delta$ contains an element fixed by $\sigma$. In other words, $X$ lies in a $\sigma$-stable $Q$-conjugate of $L$. This completes the proof of Theorem 9 .

Proof of Theorem 10. As in the hypothesis of Theorem 10, assume that $X=X(q)$ $<G$, with $q$ as in Theorem 1 and $p>N^{\prime}(X, G)$. We first aim to prove part (i) of Theorem 10 . 
By Corollary 5 , there is a proper closed connected subgroup $\bar{X}$ of $G$ containing $X$ and fixing the same subspaces of $L(G)$ as $X$. Take $\bar{X}$ to be minimal subject to these conditions.

Lemma 9.4. $\bar{X}$ is reductive.

Proof. Suppose false, so $R_{u}(\bar{X}) \neq 1$. By [BT], $\bar{X}$ lies in a parabolic subgroup $P=$ $Q R$ of $G$ (with unipotent radical $Q$ and Levi subgroup $R$ ) such that $R_{u}(\bar{X}) \leq Q$. Then by Theorem $9, X$ lies in a $Q$-conjugate of $R$, so we may take it that $X<R$.

Now $X$ fixes $L(R)$, and hence so does $\bar{X}$. But $N_{Q R}(L(R))^{0}=R$ : for if not, $N_{Q R}(L(R))^{0}=Q_{0} R$ with $Q_{0}$ a nontrivial connected subgroup of $Q$. Then $L\left(Q_{0}\right)$ commutes with $L(R)$. However, $C_{L(G)}(L(R)) \cap L(Q)=0$, since if $T$ is a maximal torus of $R$, then $C_{L(G)}(L(R)) \subseteq C_{L(G)}(L(T))=L(T)$ by 1.11 ; this contradicts the previous sentence. Therefore $\bar{X} \leq N_{Q R}(L(R))^{0}=R$. But this means that $R_{u}(\bar{X}) \leq R$, which is absurd.

By the minimality of $\bar{X}$ we have $\bar{X}^{\prime}=\bar{X}$, and hence $\bar{X}$ is semisimple. Write $\bar{X}=\bar{X}_{1} \ldots \bar{X}_{t}$, a commuting product of connected simple groups $\bar{X}_{i}$.

Lemma 9.5. All the $\bar{X}_{i}$ are of the same type as $X$.

Proof. Since $X$ and $\bar{X}$ fix the same subspaces of $L(G), X$ must be irreducible on each $\bar{X}_{i}$-composition factor of $L\left(\bar{X}_{i}\right)$ (for all $i$ ). Hence by Theorem 4 , for each $i$, either $X$ and $\bar{X}_{i}$ are of the same type, or $\left(\bar{X}_{i}, X, p\right)$ is $\left(B_{n}\right.$ or $\left.C_{n}, D_{n}^{\epsilon}(q), 2\right)$ or $\left(B_{3}\right.$ or $\left.C_{3}, G_{2}(q), 2\right)$. In each of these exceptional cases, the projection of $X$ in $\bar{X}_{i}$ lies in a connected simple subgroup of $\bar{X}_{i}$ of the same type as $X$ (as this projection is determined up to conjugacy in $\bar{X}_{i}$ ). Hence the result follows by the minimality of $\bar{X}$.

We now have

$$
X<\bar{X}_{1} \ldots \bar{X}_{t}
$$

with each $\bar{X}_{i}$ connected and simple of the same type as $X$.

We now argue that $X<\tilde{X} \leq \bar{X}_{1} \ldots \bar{X}_{t}$, where $\tilde{X}$ is connected and simple of the same type as $X$. This is clear if $t=1$, so suppose $t>1$. Let $\phi_{i}: X \rightarrow \bar{X}_{i} / Z\left(\bar{X}_{i}\right)$ be the $i^{\text {th }}$ projection map.

Let $Y$ be a simple algebraic group of the same type as $X$, and containing $X$. If $Y$ is classical, then [ST1] implies that each $\phi_{i}$ extends to a morphism $\hat{\phi}_{i}: Y \rightarrow$ $\bar{X}_{i} / Z\left(\bar{X}_{i}\right)$; and if not, then as $t>1$, we have $X=G_{2}(q)$, and the same conclusion follows from [LS3, 5.1]. Now define

$$
\hat{X}=\left\{y \hat{\phi}_{1} \ldots y \hat{\phi}_{t}: y \in Y\right\}
$$

and take $\tilde{X}$ to be the connected preimage of $\hat{X}$ in $X<\bar{X}_{1} \ldots \bar{X}_{t}$. Then $\tilde{X}$ is a closed, connected, simple subgroup of $G$ of the same type as $X$, and containing $X$. Moreover $\tilde{X} \leq \bar{X}$, so by minimality, $\tilde{X}=\bar{X}$. This completes the proof of the first part of Theorem 10(i).

For the last part of Theorem $10(\mathrm{i})$, we must show that $C_{G}(X)^{0}=C_{G}(\tilde{X})^{0}$. This is obvious if $C_{G}(X)^{0}=1$, so assume $C_{G}(X)^{0} \neq 1$.

We now argue that $C_{G}(X)^{0}$ is reductive. The proof of this follows closely that of [LS2, Theorem 4.1]. Let $C=C_{G}(X)^{0}$, and suppose $U=R_{u}(C) \neq 1$. Then $X C$ lies in a parabolic subgroup $P=Q L$, with $U \leq Q$. Certainly $X$ fixes $L(P)$, hence so does $\tilde{X}$, and therefore $\tilde{X}<P$. Then by Theorem 9 , we may assume 
that $\tilde{X} \leq L$. Now Lemmas $4.2-4.4$ of [LS2] show that there exists $l \in L$ such that $\bar{w}_{0} l$ normalizes $\tilde{X}$ (where $\bar{w}_{0}$ is an automorphism of $G$ normalizing a maximal torus of $L$ and inducing -1 on $\Sigma(G)$ ). Using [LS3, 5.1], we may take it that $\bar{w}_{0} l$ also normalizes $X$. But now we have $1 \neq\left|C_{Q}(X)\right|=\left|C_{Q^{\bar{w}_{0} l}}\left(X^{\bar{w}_{0} l}\right)\right|=\left|C_{Q^{-}}(X)\right|$, where $Q^{-}$is the unipotent radical of the parabolic opposite to $P$. This gives $1 \neq C_{Q^{-}}(X) \leq Q^{-} \cap P=1$, a contradiction.

Thus $C_{G}(X)^{0}$ is reductive. Let $T$ be a torus in $C_{G}(X)^{0}$. Then $R=C_{G}(T)$ is a Levi subgroup of $G$ containing $X$. Now $R=P \cap P^{-}$, the intersection of a parabolic $P$ and its opposite $P^{-}$. Hence $X$ fixes $L(P)$ and $L\left(P^{-}\right)$, and therefore so does $\tilde{X}$. Since $N_{G}(L(P))=P$ and $N_{G}\left(L\left(P^{-}\right)\right)=P^{-}$, we deduce that $\tilde{X} \leq P \cap P^{-}=$ $R$. Thus $\tilde{X}$ centralizes $T$. The reductive group $C_{G}(X)^{0}$ is generated by tori, so we conclude that $C_{G}(X)^{0}=C_{G}(\tilde{X})^{0}$, as required. This completes the proof of Theorem 10(i).

Now we prove part (ii) of Theorem 10. Suppose that $X=X(q)<G$, with $q$ as in Theorem 1. By Theorem 1, we can choose a $\sigma$-stable connected subgroup $\bar{X}$ of $G$, minimal subject to containing $X$ and fixing the same subspaces as $X$ of every $G$-composition factor of $L(G)$.

We claim that $\bar{X}$ is reductive. Suppose false, so that by [BT], $\bar{X}$ lies in a $\sigma$-stable parabolic $P=Q R$ of $G$, with $R_{u}(\bar{X}) \leq Q$; then by Theorem $9, X$ lies in a $\sigma$-stable $Q$-conjugate of $R$. If $L(G)$ is $G$-irreducible, the proof of Lemma 9.4 shows that $R_{u}(\bar{X}) \leq R$, a contradiction. Otherwise, by 1.10 , we have $(G, p)=\left(E_{7}, 2\right),\left(E_{6}, 3\right)$, $\left(F_{4}, 2\right)$ or $\left(G_{2}, 3\right)$. In the first two cases we may assume that $G$ is simply connected, so that $G$ is irreducible on $L(G) / Z(L(G))$ and $\bar{X}$ fixes each $X$-invariant subspace of $L(G) / Z(L(G))$. As $L(R)>L(T)>Z(L(G))$ (where $T$ is a maximal torus of $R$ ), we conclude that $X$ fixes $L(R)$, and at this point the proof of 9.4 works as before to give the claim. For the $F_{4}$ and $G_{2}$ cases, by the prime restrictions $p>N^{\prime}(X, G)$, the only possibility is that $(G, p, X)=\left(F_{4}, 2, A_{3}^{\epsilon}(q)\right)$. Then $Q L=Q B_{3}$ or $Q C_{3}$. Let $I$ be the 26-dimensional ideal of $L(G)$ generated by short root elements. We may take it that $X<L$; hence $X$ fixes $L\left(B_{3}\right) \cap I$ or $L\left(C_{3}\right) \cap I$. Therefore $\bar{X}$ lies in the stabilizer of one of these subspaces, hence lies in a subgroup $B_{3} \tilde{A}_{1}$ or $C_{3} A_{1}$ of $F_{4}$. Moreover, $X$, hence $\bar{X}$, fixes every subspace of $L\left(\tilde{A}_{1}\right)$ or $L\left(A_{1}\right)$, from which it follows that $\bar{X}<B_{3} T_{1}$ or $C_{3} T_{1}$, whence $\bar{X}<L$.

Thus $\bar{X}$ is reductive. By minimality, $\bar{X}$ is semisimple, say $\bar{X}=\bar{X}_{1} \ldots \bar{X}_{t}$ with each $\bar{X}_{i}$ simple. We claim also that each $\bar{X}_{i}$ is of the same type as $X$. As in the proof of Lemma 9.5, this follows from Theorem 4, unless for some $i,\left(\bar{X}_{i}, X, p\right)$ is $\left(B_{n}\right.$ or $\left.C_{n}, D_{n}^{\epsilon}(q), 2\right)$ or $\left(B_{3}\right.$ or $\left.C_{3}, G_{2}(q), 2\right)$. Assume then that one of the latter two cases holds. The fact that $p>N^{\prime}(X, G)$ implies that the first case holds with $t=1, n \geq 4$. But then $X$ lies in a $\sigma$-stable subgroup $D_{n}$ of $\bar{X}$, contrary to the minimality of $\bar{X}$. This proves our claim.

To finish the proof of Theorem $10(\mathrm{ii})$, we need to show that $C_{G}(X)^{0}=C_{G}(\bar{X})^{0}$. We showed for part (i) of Theorem 10 that $C_{G}(X)^{0}=C_{G}(\tilde{X})^{0}$, where $\tilde{X}$ is a suitable simple connected diagonal subgroup of $\bar{X}$ of type $X$. Define $C C^{0}(X)=$ $C_{G}\left(C_{G}(X)^{0}\right)^{0}$. This group contains $\tilde{X}$ and is $\sigma$-stable. If

$$
Y=\left\langle\tilde{X}^{\sigma^{i}}: \text { all } i\right\rangle
$$

then $Y$ lies in $\bar{X}$ and is $\sigma$-stable; hence $Y=\bar{X}$ by the minimality of $\bar{X}$, and consequently $C C^{0}(X)$ contains $\bar{X}$. It follows that $C_{G}(X)^{0} \leq C_{G}(\bar{X})^{0}$. The reverse inclusion is trivial, so $C_{G}(X)^{0}=C_{G}(\bar{X})^{0}$, as required. 


\section{Proof of TheOrem 11}

In this final section we prove Theorem 11. Let $C$ be a finite classical group in characteristic $p$ with usual module $V$, and suppose $X, Y$ are simple groups of Lie type in characteristic $p$, not of the same type, such that $X<Y<C$ and $X$ is absolutely irreducible on $V$. Suppose also that if $Y$ is of exceptional type, then $X=X(q)$ with $q$ as in the hypothesis of Theorem 11 .

First observe that by [ST1], the embedding $Y<C$ lifts to an embedding $\bar{Y} \leq \bar{C}$ of corresponding simple algebraic groups. Let $\bar{V}$ be the usual module for $\bar{C}$. If $\bar{Y}$ is classical, then again by [ST1], $X<Y$ lifts to an embedding $\bar{X}<\bar{Y}$, giving the required conclusion.

Thus we assume that $\bar{Y}$ is exceptional. By Theorem $1, X<Z \leq \bar{Y}$ for some closed connected subgroup $Z$ of $\bar{Y}$ fixing the same subspaces of $L(\overline{\bar{Y}})$ as $X$. By Theorem 4, $X$ is reducible on some $\bar{Y}$-composition factor of $L(\bar{Y})$, and so $Z<\bar{Y}$. Now $X$, and hence $Z$, is irreducible on $\bar{V}$, so $Z$ is semisimple.

At this point we have

$$
X<Z<\bar{Y}<\bar{C} .
$$

Now we apply [Se1, Theorem 1]; this says that the triple $(Z, \bar{Y}, \bar{C})$ is one of those in Table 1 of [Se1]. Inspection of that table (recalling that $\bar{Y}$ is exceptional) shows that $Z$ must be simple. If $Z$ is classical, then [ST1] again implies that $X<\bar{X} \leq Z$ for some simple connected group $\bar{X}$ of the same type as $X$, as required. If $Z$ is exceptional and of the same type as $X$, we take $\bar{X}=Z$. Finally, if $Z$ is exceptional and not of the same type as $X$, we replace $\bar{Y}$ with $Z$ and repeat the argument; eventually we obtain a suitable group $\bar{X}$. This completes the proof of Theorem 11 .

\section{REFERENCES}

[An] H.H. Andersen, "Filtrations of cohomology modules for Chevalley groups", Ann. Sci. Ec. Norm. Sup. 16 (1983), 495-528. MR 85k:20128

[AJL] H.H. Andersen, J. Jorgensen and P. Landrock, "The projective indecomposable modules of $S L\left(2, p^{n}\right)$ ", Proc. London Math. Soc. 46 (1983), 38-52. MR 84f:20044

[As] M. Aschbacher, "On finite groups of Lie type and odd characteristic", J. Algebra 66 (1980), 400-424. MR 81k:20023

[Bor1] A. Borel, Linear Algebraic Groups, Second Edition, Springer-Verlag, New York, 1991. MR 92d:20001

[Bor2] A. Borel, "Properties and linear representations of Chevalley groups", in: Seminar on algebraic groups and related topics (ed. A. Borel et al.), Lecture Notes in Math. 131, Springer, Berlin, 1970, pp. 1-55. MR 41:3484

[BT] A. Borel and J. Tits, "Éléments unipotents et sous-groupes paraboliques de groupes réductifs", Invent. Math. 12 (1971), 95-104. MR 45:3419

[Bo] N. Bourbaki, Groupes et algèbres de Lie (Chapters 4,5,6), Hermann, Paris, 1968. MR 39: 1590

[BW] N. Burgoyne and C. Williamson, "Some computations involving simple Lie algebras", Proc. 2nd. Symp. Symbolic and Algebraic Manipulation, (ed. S. Petrick), N.Y. Assoc. Computing Machinery, 1971, 162-171.

[CPSK] E. Cline, B. Parshall, L. Scott and W. van der Kallen, "Rational and generic cohomology", Invent. Math. 39 (1977), 143-163. MR 55:12737

[CG] A.M. Cohen and R. Griess, "On finite simple subgroups of the complex Lie group of type E8", Proc. Symp. Pure Math. 47 (1987), 367-405. MR 90a:20089

[DL] D. Deriziotis and M.W. Liebeck, "Centralizers of semisimple elements in finite twisted groups of Lie type", J. London Math. Soc. 31 (1985), 48-54. MR 87e:20087

[GS] P. Gilkey and G.M. Seitz, "Some representations of exceptional Lie algebras", Geom. Ded. 25 (1988), 407-416. MR 89h:20056 
[GL] D. Gorenstein and R. Lyons, "The local structure of finite groups of characterstic 2 type", Memoirs Amer. Math. Soc. 276 (1983). MR 84g:20025

[Ja] J.C. Jantzen, "Low dimensional representations of reductive groups are semisimple", in Algebraic groups and related subjects; a volume in honour of R.W. Richardson (eds. G.I. Lehrer et al.), Austral. Math. Soc. Lecture Note Series, 1996.

[JP] W. Jones and B. Parshall, "On the 1-cohomology of finite groups of Lie type", Proc. Conf. on Finite Groups (eds. W. Scott and F. Gross), Academic Press, 1976, pp. 313-328. MR 53:8272

[LT] R. Lawther and D.M. Testerman, " $A_{1}$ subgroups of exceptional algebraic groups", Trans. Amer. Math. Soc., to appear.

[Li] M.W. Liebeck, "On the orders of maximal subgroups of the finite classical groups", Proc. London Math. Soc. 50 (1985), 426-446. MR 87a:20046

[LS1] M.W. Liebeck and G.M. Seitz, "Maximal subgroups of exceptional groups of Lie type, finite and algebraic", Geom. Dedicata 36 (1990), 353-387. MR 91f:20032

[LS2] M.W. Liebeck and G.M. Seitz, "Reductive subgroups of exceptional algebraic groups", Memoirs Amer. Math. Soc., No. 580 (1996). MR 96i:20059

[LS3] M.W. Liebeck and G.M. Seitz, "Subgroups generated by root elements in groups of Lie type", Ann. of Math. 139 (1994), 293-361. MR 95d:20078

[LSS] M.W. Liebeck, J. Saxl and G.M. Seitz, "Subgroups of maximal rank in finite exceptional groups of Lie type", Proc. London Math. Soc. 65 (1992), 297-325. MR 93e:20046

[LST] M.W. Liebeck, J. Saxl and D.M. Testerman, "Simple subgroups of large rank in groups of Lie type", Proc. London Math. Soc. 72 (1996), 425-457. MR 96k:20087

[Pr] O. Premet, "Weights of infinitesimally irreducible representations of Chevalley groups over a field of prime characteristic", Math. USSR Sbornik 61 (1988), 167-183. MR 88h:20051

[Se1] G.M. Seitz, "The maximal subgroups of classical algebraic groups", Mem. Amer. Math. Soc., No. 365 (1987). MR 88g:20092

[Se2] G.M. Seitz, "Maximal subgroups of exceptional algebraic groups", Memoirs Amer. Math. Soc., No. 441 (1991). MR 91g:20038

[Se3] G.M. Seitz, "Representations and maximal subgroups of finite groups of Lie type", Geom. Dedicata 25 (1988), 391-406. MR 89d:20035

[Se4] G.M. Seitz, "Flag-transitive subgroups of Chevalley groups", Annals of Math. 97 (1973), 27-56. MR 49:5201

[ST1] G.M. Seitz and D.M. Testerman, "Extending morphisms from finite to algebraic groups", J. Algebra 131 (1990), 559-574. MR 91f:20054

[ST2] G.M. Seitz and D.M. Testerman, "Subgroups of type $A_{1}$ containing semiregular unipotent elements", J. Algebra 196 (1997), 595-619. CMP 98:02

[Sin] P. Sin, "Extensions of simple modules for $S L_{3}\left(2^{n}\right)$ and $S U_{3}\left(2^{n}\right)$ ", Proc. London Math. Soc. 65 (1992), 265-296. MR 93e:20021

[SS] T.A. Springer and R. Steinberg, "Conjugacy classes", in: Seminar on algebraic groups and related topics (ed. A. Borel et al.), Lecture Notes in Math. 131, Springer, Berlin, 1970, pp. 168-266. MR 42:3091

[St] R. Steinberg, Lectures on Chevalley Groups, notes by J. Faulkner and R. Wilson, Yale University (1968). MR 57:6215

[Te1] D.M. Testerman, "Irreducible subgroups of exceptional algebraic groups", Mem. Amer. Math. Soc., No. 390 (1988). MR 90a:20082

[Te2] D.M. Testerman, "A construction of certain maximal subgroups of the algebraic groups $E_{6}$ and $F_{4} ", J$. Algebra 122 (1989), 299-322. MR 90d:20082

Department of Mathematics, Imperial College, London SW7 2BZ, United Kingdom

E-mail address: m.liebeck@ic.ac.uk

Department of Mathematics, University of Oregon, Eugene, Oregon 97403

E-mail address: seitz@math.uoregon.edu 